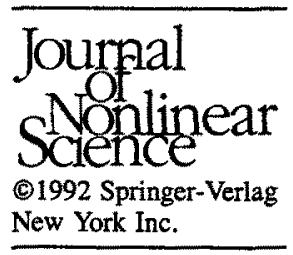

\title{
Beyond All Orders: Singular Perturbations in a Mapping
}

\author{
C. Amick ${ }^{1,2}$, E. S. C. Ching ${ }^{1}$, L. P. Kadanoff ${ }^{1}$, and V. Rom-Kedar ${ }^{1}$ \\ ${ }^{1}$ Computational and Applied Mathematics Program, The Department of Mathematics, The \\ University of Chicago, Chicago, IL 60637, USA
}

Received February 2, 1990; accepted for publication October 25, 1991

Communicated by Philip Holmes

Summary. We consider a family of $q$-dimensional ( $q>1$ ), volume-preserving maps depending on a small parameter $\varepsilon$. As $\varepsilon \rightarrow 0^{+}$these maps asymptote to flows which attain a heteroclinic connection. We show that for small $\varepsilon$ the heteroclinic connection breaks up and that the splitting between its components scales with $\varepsilon$ like $\varepsilon^{\gamma} \exp [-\beta / \varepsilon]$. We estimate $\beta$ using the singularities of the $\varepsilon \rightarrow 0^{+}$heteroclinic orbit in the complex plane. We then estimate $\gamma$ using linearization about orbits in the complex plane. These estimates, as well as the assertions regarding the behavior of the functions in the complex plane, are supported by our numerical calculations.

Key words. break-up of heteroclinic connection, exponentially small splitting of separatrices, singular perturbation

\section{Introduction}

\section{A. The Problem}

By now, there is a well-developed literature on singular perturbations and how they affect the solution of ordinary ${ }^{1}$ and partial ${ }^{2}$ differential equations. In a typical singular perturbation problem, there is a small parameter $\varepsilon$ which appears in the differential equation as a multiplier of the highest-order derivative. No matter how small $\varepsilon$ might be, this term can nonetheless have a very substantial effect upon the solution.

2 Deceased. 
In this paper, we consider singular perturbations in the context of a mapping problem

$$
x_{j+q}=x_{j}+\varepsilon g\left(x_{j+p}\right) .
$$

Here the $x_{j}$ 's are real numbers, $q$ and $p$ are relative prime integers which obey

$$
0<p<q
$$

the parameter $\varepsilon$ is a small positive number, while $g(x)$ is an entire function, positive in the interior of the interval $[0,1]$, and vanishing at the endpoints

$$
g(0)=g(1)=0 .
$$

We also demand that the derivative of $g$ not vanish at the endpoints

$$
g^{\prime}(0)>0, \quad g^{\prime}(1)<0 .
$$

This problem can be re-expressed as a volume-preserving map in a q-dimensional phase space, in which the basic variable is

$$
X_{j}=\left(x_{j}, x_{j+1}, \ldots, x_{j+q-1}\right)
$$

and the map is

$$
X_{j+1}=G\left(X_{j}\right)
$$

with

$$
G\left(x_{0}, x_{1}, \ldots, x_{q-2}, x_{q-1}\right)=\left(x_{1}, x_{2}, \ldots, x_{q-1}, x_{0}+\varepsilon g\left(x_{p}\right)\right) .
$$

(Here and below we use a notation in which capital symbols refer to $q$-dimensional vectors and lower-case symbols to scalars and components of these vectors.) For $q=$ 2 and 3, the map (1.6) may be thought of as the Poincare map of a time-periodic flow. Indeed, our initial interest in this problem arose when we studied a simplified version of the $A B C$ map, ${ }^{3}$ which mimics the dynamics of the time-periodic flow.

Notice that the inverse of this map is another mapping problem of essentially the same form as (1.7), namely,

$$
G^{-1}\left(x_{1}, x_{2}, \ldots, x_{q}\right)=\left(x_{q}-\varepsilon g\left(x_{p}\right), x_{1}, x_{2}, \ldots, x_{q-1}\right) .
$$

This inverse map can be reduced to exactly the same form as (1.1) with $\varepsilon<0$ by making the change of variables $y_{j}=x_{q-j}$ and replacing $p$ by $p^{\prime}=q-p$.

In this $q$-dimensional space there exist two unstable hyperbolic fixed points $0=$ $(0,0, \ldots, 0)$ and $1=(1,1, \ldots, 1)$. By the stable manifold theorem, these fixed points have smooth stable and unstable manifolds. ${ }^{4,5,6}$ The dimensions of these manifolds depend on $q$ and $p$ and are always greater than or equal to 1 .

We study a one-dimensional submanifold of the unstable manifold of 0 , which we call the unstable curve, and a one-dimensional submanifold of the stable manifold of 1 , called the stable curve. These curves are defined to be the invariant manifolds on which orbits leave (resp. approach) the neighborhood of the fixed point 0 (resp. 1) most rapidly. The parameterized unstable and stable curves are denoted by $U(t)$ and 
$S(r)$, respectively. Here $r$ and $t$ are, for now, real variables in $[0, \infty)$, and $U(0)=0$, $S(0)=1$. The specific parameterization we choose is described in the next section.

The limit $\varepsilon \rightarrow 0^{+}$of (1.6) is highly singular. On the one hand, when $\varepsilon=0,(1.1)$ simply has a periodic solution $x_{j+q}=x_{j}$, and the hyperbolic structure of the fixed points is destroyed. If one defines $\alpha=j \bmod q$, in this case, there is no coupling between $x_{j}$ 's with different $\alpha$-values. Hence, the problem falls apart into $q$ decoupled and degenerate problems. For small $\varepsilon$, these problems become coupled. Indeed, if one starts with generically chosen $x_{j}(j=1,2, \ldots, q)$ of order unity, and then one uses the map to advance $j$ by an amount of order $\varepsilon^{-1} \gg 1$, one finds tha. the new $x_{j}$ 's will differ from their starting values by an amount of order unity. Thus, the singular perturbation $\varepsilon$ effectively lifts the degeneracy of the $\varepsilon=0$ problem. In this paper, we study the flows from the two fixed points in the limit $\varepsilon \rightarrow 0^{+}$. As one might expect, after many iterations, these flows show a nontrivial structure in their $\varepsilon$-dependence.

\section{B. Outline of Results}

Below, we shall develop the qualitative properties of these curves using both analytical and numerical methods. The structure which arises for small $\varepsilon$ is both interesting and elegant. The result for $q=3, p=2$ is depicted in the cartoon of Figure 1 .

As $t$ increases, $U(t)$ moves uniformly away from 0 and toward 1. As it gets close to 1 it begins to spiral outward and moves away from this fixed point. We denote the distance of $U(t)$ from 1 by $\delta_{1}(t)$ (see Figure 1). There is a distance of closest

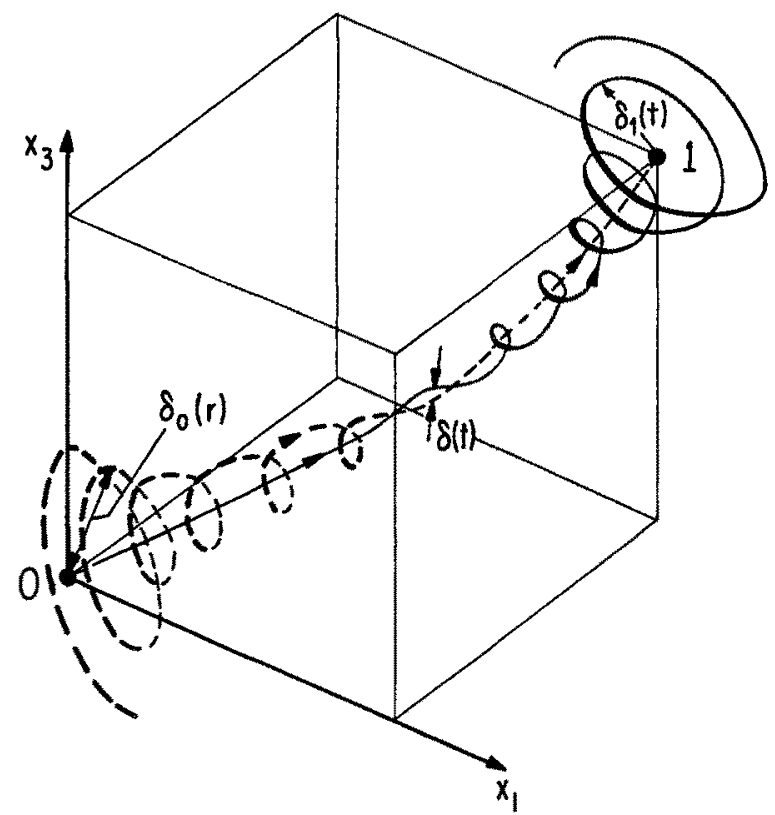

Fig. 1. Cartoon of the stable and unstable curves for $q=$ $3, p=2$. The solid line is the unstable curve, and the dashed line is the stable curve. The quantities $\delta_{0}(r), \delta_{1}(t)$, and $\delta(t)$ are also shown in the figure. 
approach ${ }^{7}$ to 1 which we call $\delta_{1}$. Conversely, $S(r)$ initially moves away from 1 . This curve approaches $U(t)$ very closely in an intermediate region between the two fixed points. The distance between the curves in this region at the point $U(t)$ is denoted by $\delta(t)$. Finally, $S(r)$ gets quite close to 0 and then spirals away from that fixed point. We denote the distance of $S(r)$ from 0 by $\delta_{0}(r)$, and its minimum ${ }^{7}$ by $\delta_{0}$.

There can be some qualitative differences between even and odd $q$. These cases correspond respectively to orientation-preserving and orientation-reversing maps. In the even $q$ case, there is the possibility that the leading-order behavior is the repeated crossing (or almost crossing) of invariant curves. For odd $q$, the curves, to the leading order, circle around one another.

In this paper, we shall be most concerned with estimating the "miss distances" $\delta_{0}, \delta_{1}$ and $\delta(t)$ in the limit $\varepsilon \rightarrow 0^{+}$. As we shall see, these distances have an order which is smaller than any power of $\varepsilon$. In fact, far enough from the fixed points 0 and 1 (where $t=O(1)$ ), $\delta(t)$ takes the form

$$
\delta(t) \sim A e^{-2 \pi \phi_{s} / \varepsilon} \varepsilon^{\gamma}, \quad t \sim 1 .
$$

In Table 1 , we summarize the results of the paper by listing the values of $\beta=2 \pi \phi_{s}$ and $\gamma$ obtained for various different functions $g(x)$.

Notice that this miss distance can be fantastically small. For $g(x)=x(1-x), \varepsilon=$ $\frac{1}{2}$, and $q=3$, we estimate $e^{-2 \pi \phi_{s} / \varepsilon} \sim 10^{-17}$ and find numerically $A=2.5 \times 10^{3}$, so $\delta(1) \sim 10^{-14}$ ! Clearly, our numerical work will have to be rather careful.

From our analysis, $\delta_{0}$ and $\delta_{1}$ will be somewhat less extreme. We found these for $q=3$ and estimated

$$
\delta_{0} \sim \delta_{1} \sim \delta(1)^{2 / 3}
$$

In general, ${ }^{8}$

$$
\begin{aligned}
& \delta_{0} \sim \delta(1)^{\eta_{0}(p, q)}, \\
& \delta_{1} \sim \delta(1)^{\eta_{I}(p, q)},
\end{aligned}
$$

\begin{tabular}{|c|c|c|c|}
\hline \multicolumn{2}{|c|}{ Function, $g(x)$} & $\beta=2 \pi \phi_{s}$ & $\gamma$ \\
\hline \multicolumn{2}{|c|}{$\begin{aligned} & x\left(1-x^{m}\right) \\
m= & \text { positive integer }\end{aligned}$} & $\frac{2 \pi^{2}}{m}$ & $-\frac{1}{m}+\left(1+\frac{1}{m}\right) \cos \frac{2 \pi p}{q}$ \\
\hline$x(1-x)(1+a x)$ & $\begin{array}{l}0<a<\infty \\
-1<a<0\end{array}$ & $\begin{array}{c}\frac{2 \pi^{2}}{1+a} \\
2 \pi^{2}\end{array}$ & $-\frac{1}{2}+\frac{3}{2} \cos \frac{2 \pi p}{q}$ \\
\hline \multicolumn{2}{|c|}{$\frac{\sin \pi x}{\pi}$} & $\pi^{2}$ & $\cos \frac{2 \pi p}{q}$ \\
\hline
\end{tabular}

to lowest order in $\varepsilon$. The values of $\eta_{0}$ and $\eta_{1}$ are given in Table 2 .

Table 1 Expressions for the coefficients in the asymptotic expansion of the miss distance $\delta(t)$ where $t$ is of order one. 
Table 2 Theoretical and numerical values of $\eta_{0}(q, p)$ and $\eta_{1}(q, p)$ for $g(x)=x\left(1-x^{m}\right)$.

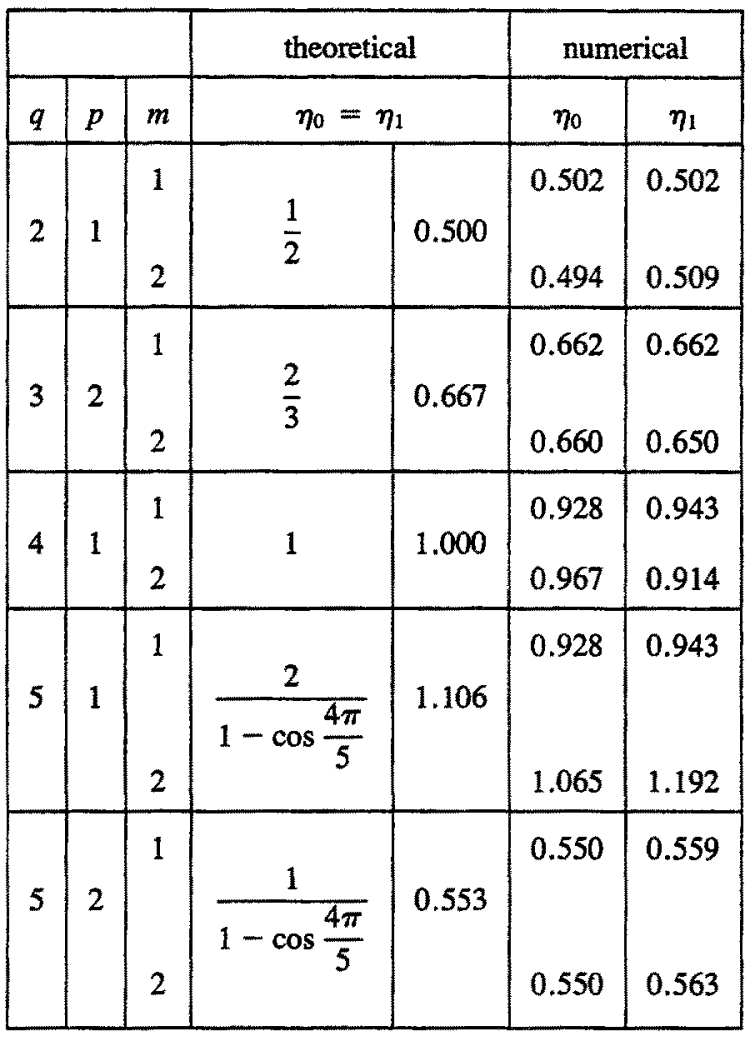

We have already summarized our results for the mapping in the real domain. However, to obtain such results in solid form, we must proceed through the complex plane. Here we summarize our major results in the complex plane.

1. We state the first result in a proposition (see Appendix A for the proof).

Proposition 1. For every $\varepsilon>0$, there exist unique entire vector-valued functions $U(t)$ and $S(r(t)),\left(U: C \rightarrow C^{q}\right.$ and $\left.S: C \rightarrow C^{q}\right)$, which are solutions to the mapping problem in the sense that

$$
U(\Lambda t)=G(U(t))
$$

and

$$
S(r / \Gamma)=G(S(r))
$$

with the conditions that

$$
\begin{aligned}
U(0) & =0, \quad S(0)=1 \\
\lim _{t \rightarrow 0} \frac{U(t)}{t} & =\left(1, \Lambda, \ldots, \Lambda^{q-1}\right), \quad \lim _{r \rightarrow 0} \frac{S(r)-S(0)}{r}=-\left(1, \Gamma^{-1}, \ldots, \Gamma^{-q+1}\right) .
\end{aligned}
$$


Here $\Lambda$ and $\Gamma$ are respectively the real solutions to

$$
\begin{aligned}
& \Lambda^{q}=1+\varepsilon g^{\prime}(0) \Lambda^{p}, \\
& \Gamma^{q}=1-\varepsilon g^{\prime}(1) \Gamma^{q-p},
\end{aligned}
$$

with the largest magnitude.

2. We claim that $U(t)$ and $S(r(t))$ approach a unique limit $U_{0}(t)$, as $\varepsilon \rightarrow 0^{+}$, everywhere in a finite open region, $R_{\bar{\phi}}$, of $\ln t$ bounded by

$$
-\tilde{\phi}<\operatorname{Im}(\ln t)<\tilde{\phi}
$$

where $\tilde{\phi}<\phi_{s}$. The function $U_{0}(t)$ is analytic in $R_{\tilde{\phi}} ; \phi_{s}$ is defined as the smallest positive phase for which $U_{0}\left(|t| e^{i \phi_{s}}\right)$ is singular for some finite value of $|t|$. Supporting evidence for this claim is presented in Appendix D.

3. Further, we claim that there exists an analytic function $r(t)$ in $R_{\tilde{\phi}}$, which obeys $r(\Lambda t)=r(t) / \Gamma$, and for which the quantity

$$
\Delta(t)=S(r(t))-U(t)
$$

is quite small in the region $R_{\tilde{\phi}}$. Specifically, $\delta(t)=\|\Delta(t)\|$ goes uniformly to zero as $\varepsilon \rightarrow 0^{+}$(see Appendix D).

4. We claim that not only does $\|\Delta(t)\|$ go to zero but that in $R_{\tilde{\phi}}$,

$$
\|\Delta(t)\| \exp (2 \pi \phi / \varepsilon) \rightarrow 0, \quad \text { as } \varepsilon \rightarrow 0^{+} .
$$

This limit is not uniform in $\phi$, which is the phase of $t$

$$
\phi=\operatorname{Im}(\ln t) \text {. }
$$

The argument for this result is developed in the first part of Appendix D.

5. Finally, we conjecture that

$$
\|\Delta(t)\| \exp (2 \pi h(\phi) / \varepsilon)
$$

is bounded by a power of $\varepsilon^{-1}$ for all sufficiently small $\varepsilon$ in each such open region, $R_{\tilde{\phi}}$. Here the bound is defined by

$$
h(\phi)=\phi_{s}-|\phi| .
$$

The heuristic argument for this conjecture is developed in the second part of Appendix D. It is supported by numerical evidence presented in Section IV.

All of our work depends on two essentially small parameters, which are related but which play different roles. The first is $\varepsilon$, which is actually not so small. If we work at $\varepsilon=\frac{1}{2}, g^{\prime}(0)=1, q=3$, and $p=1$, for example, there is a $0.11 \%$ correction to the first-order solution of (1.13), $\Lambda=1+\varepsilon / q=\frac{7}{6}$. The other small parameter is $\xi(\phi)=\exp (-2 \pi h(\phi) / \varepsilon)$, which is exponential in $\varepsilon^{-1}$ and can be very small indeed. In our chosen example with $g(x)=x(1-x)$, it has a value of $\sim 10^{-17}$ on the real axis. For our purposes, we wish to keep only the lowest-order values in this other parameter. We have argued that $\|\Delta(t)\|$ is proportional to this parameter. Hence, expansions in $\|\Delta(t)\|$ are likely to be quite accurate. 


\section{Relation to Other Work}

The existence of a heteroclinic connection is associated with important physical phenomena such as solitons ${ }^{9}$ (when the map represents a reduction of PDE) and flow barriers ${ }^{10}$ (in two dimensions, $q=2$ ). The remnants of this connection, when $\varepsilon$ is finite, constitute transient behavior. ${ }^{9,10}$ Their possible structure is described in Section II.D. In this paper we show that inherently, even when the $q$-dimensional limiting flow attains a heteroclinic connection, the map does not. This implies, for example, that the exponentially small splitting of separatrices seen in a numerical simulation of a system of ODE's may be the result of the discretization scheme rather than the nonintegrability of the ODE's. Ablowitz and Herbst ${ }^{11}$ have demonstrated that this phenomenon occurs for the homoclinic structures of the nonlinear Schrödinger equation. Hockett ${ }^{12}$ proved that an Euler discretization of an integrable central force problem leads to chaotic dynamics. This may be associated with the breaking up of homoclinic connection (with real fixed point at infinity).

The phenomenon of exponentially small splitting of separatrices for ODE's was first observed by Poincare ${ }^{13}$ about a hundred years ago. He noted that an integrable two-dimensional flow (for example, resonance bands) perturbed by a fast oscillatory forcing, will typically exhibit such a splitting. Nekhoroshev ${ }^{14}$ and Neishtadt ${ }^{15}$ established rough upper estimates on the exponentially long escape times associated with the small splitting. Holmes et al. ${ }^{16}$ established lower and upper bounds on the exponentially small distance between the split separatrices for a class of fast oscillatory perturbations with sufficiently small amplitude. Zaslavskii et al. ${ }^{17}$ reviewed the dynamics on stochastic webs, where exponentially small splitting implies diffusionlike motion of the particles.

In this paper we relate special solutions of the mappings to heteroclinic solutions of the limiting flows. In spirit, we follow the program described by Tanveer for solving singular perturbation problems. Tanveer ${ }^{18}$ has summarized the method of Kruskal and Segur ${ }^{19}$ for attacking problems roughly like this. He suggests that one solves for the flow at infinitesimal $\varepsilon$ including all the flexibility allowed by general values of constants of integration. Then one examines the solution for singularities in the complex plane. These singular regions should be used as places at which one can patch together different pieces of solutions with different constants of integration. To perform the patching, one requires a more accurate solution in the neighborhood of the singularity: one which includes finite- $\varepsilon$ effects. At this point, one has to examine the behavior of the expansion in $\varepsilon$ in the patching region. In general, the series expansion diverges near the singularity. One has to choose a special patching region or a pass in the complex plane to ensure that the matching is done using the leading-order term of a converging series. Theoretically, it is usually very hard to prove that the above condition is satisfied. In Appendix D, we formulate a set of propositions, which, if proven, constitute a proof of our claims. The assertions which we cannot prove yet are supported by numerical calculations in the complex plane. Hence, while we cannot claim rigor, we believe our theory is based on solid grounds.

Kruskal and Segur's work on dendritic solidification was followed by the work of Amick and McLeod, ${ }^{20}$ who have proved that the exponentially small estimate found by Kruskal and Segur is a valid asymptotic limit. As indicated in their paper, 
the methods developed by Amick and McLeod can be easily utilized to study the behavior of solutions of a large family of ODE's. The analogous proof of the validity of the exponentially small estimates found here, tailored to the case of difference equations (as opposed to ODE's), is yet to be derived. The formulation in Appendix $D$ may be a first step in this direction.

Lazutkin et al. ${ }^{21}$ estimated the angle between the intersecting manifolds of the standard mapping in the integrable limit. They found that this angle is exponentially small in $\varepsilon$. They estimated the exponent and the algebraic dependence on $\varepsilon$, and computed the prefactor. Their method is based upon the existence of an energy function and utilizes the form of the mapping to obtain convenient coordinate changes. Fontich and $\operatorname{Sim}^{22}$ have studied the general problem of near identity, two-dimensional conservative analytic mappings attaining hyperbolic point. Their work applies to ours in the special case of $q=2$. Using the existence of an energy function, they obtained an upper bound on the split distance in the form of $\exp \left(-2 \pi \phi_{s} / \varepsilon\right)$, and were able to verify the value of $\phi_{s}$ numerically. Our work, which applies to all $q$, is based upon an extension of the unstable curve $U(t)$, and the linearized modes about $U(t)$ to complex values of $t$.

Recently, Scheurle et al. ${ }^{23}$ constructed an example of a mapping in which the splitting distance is of the form

$$
\mid \text { splitting distance } \mid=\left(\sum_{k=-\infty}^{\infty} a_{k} \varepsilon^{k}\right) e^{-c / \varepsilon} .
$$

In particular, the splitting distance cannot be bounded, as $\varepsilon \rightarrow 0$, by any finite power of $\varepsilon$ in front of the exponential term. They conjectured that the form of (1.18) is generic and that typical estimates of the form (1.9) are valid only for a finite range of $\varepsilon$ values (namely $\gamma=\gamma(\varepsilon)$ ). We think that our case does not fall into their generic category. We suggest that the main difference between the mappings considered by us and them is the analyticity property of the stable and unstable curves (which is used to develop (1.9)). While in our example, these curves are entire functions of their arguments, in the example considered by Scheurle et al., the curves attain essential singularities for all $\varepsilon$. Our numerical study supports the prediction of $\gamma$ and $\beta$ in (1.9). However, any calculation of a finite range of values of $\varepsilon$ cannot exclude the possibility that the form of (1.18) is valid as $\varepsilon \rightarrow 0^{+}$.

\section{Outline of the Paper}

In the next section, we define the unstable and stable curves and their parameterizations. Since $\mathbf{0}$ and 1 are hyperbolic fixed points, the existence, uniqueness, and smoothness of these invariant curves follow from the stable manifold theorem. (In Appendix A, we show that the parameterized curves are entire functions of their arguments.) We discuss the singular limit $\varepsilon \rightarrow 0^{+}$and introduce the lowest-order solution in this limit with $j \varepsilon=O(1)$ where $j$ denotes the number of iterations of the map (1.6). Then, we discuss the possible behavior of the stable and unstable curves.

In Section III we perform a linear deviation analysis. We linearize the equations about the stable and unstable curves and find, to lowest order in $\varepsilon$, the linear modes. 
We establish that the coefficients of the linear modes are the only unknowns in the equation for $\delta(t)$. We derive some of their properties and present numerical calculations which support our observations.

In Section IV we employ complex analysis to estimate the coefficients of the linear modes by matching solutions near the singularities of $U_{0}(t)$. We use the lowest-order solutions in $\varepsilon$ to obtain the exponential estimate and then the next-order to obtain the algebraic correction. We then derive the estimates for $\delta_{1}$ and $\delta_{0}$. These estimates of $\delta_{1}, \delta_{0}$, and $\delta(t)$ are supported by numerical calculations. Furthermore, the numerical calculations in the complex plane also support our assertions regarding the behavior of the linear modes and the coefficients near the singularities.

In the last section, we summarize our results.

In Appendix A we prove Proposition 1. In Appendix B we expand the curves in power series. In Appendix $C$ we present the error analysis of the numerical work. In Appendix D we construct an initial framework for proving our theory.

\section{Stable and Unstable Curves}

\section{A. Definitions}

We do linear stability analysis in the neighborhood of $X=0$. Linearization of (1.6) near 0 gives

$$
V_{j+1}=D G(0) V_{j}=\left(v_{j+1}, \ldots, v_{j+q-1}, v_{j}+\varepsilon g^{\prime}(0) v_{j+p}\right) .
$$

The characteristic equation of $D G(0)$ is given by

$$
\Lambda_{\alpha}^{q}=1+\varepsilon g^{\prime}(0) \Lambda_{\alpha}^{p} .
$$

Here, $\alpha$ labels the $q$ different roots of (2.2). When $\varepsilon \geq 0$, they are all distinct. In our labeling scheme, the index $\alpha$ is given by

$$
\begin{aligned}
& \alpha=0, \pm 1, \ldots, \pm(q-1) / 2, \quad \text { for } q \text { odd, } \\
& \alpha=0, \pm 1, \ldots, \pm(q-2) / 2, q / 2, \quad \text { for } q \text { even. }
\end{aligned}
$$

To first order in $\varepsilon$, the solution to (2.2) is

$$
\Lambda_{\alpha}=\Omega^{\alpha}\left[1+\frac{\varepsilon g^{\prime}(0) \Omega^{\alpha p}}{q}\right]+O\left(\varepsilon^{2}\right)
$$

with $\Omega$ being the $q$ th root of unity

$$
\Omega=e^{2 \pi i / q} .
$$

The root with the largest magnitude is $\Lambda_{0}$, which we denote by $\Lambda$. Note that $\Lambda$ is real and greater than 1. Figure 2 shows the configuration of eigenvalues in the complex plane which arise for various values of $q$ and $p$.

For small positive values of $\varepsilon$, the roots lie inside the unit circle if $\cos (2 \pi \alpha p / q)<$ 0 and outside whenever $\cos (2 \pi \alpha p / q) \geq 0$. In the latter case, the corresponding eigenmode is unstable around zero; in the former case, it is stable. 

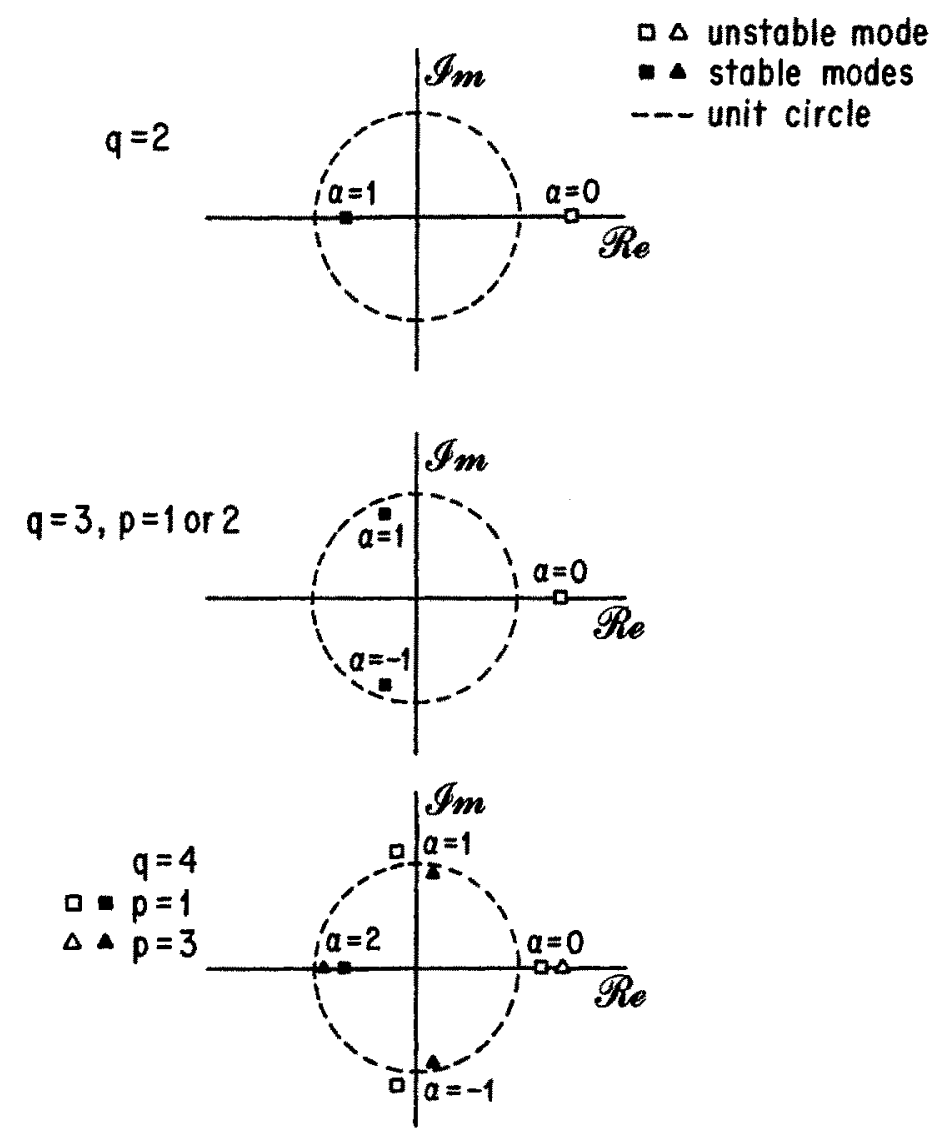

Fig. 2. Stability diagram. A schematic diagram of the eigenvalues $\Lambda_{\alpha}$ in the complex plane for various $q$ and $p$.

The eigenvector, $V_{\alpha}$, of the root $\Lambda_{\alpha}$ is given by

$$
V_{\alpha}=\left(1, \Lambda_{\alpha}, \ldots, \Lambda_{\alpha}^{q-1}\right),
$$

and solutions to (2.1) are given by linear combination of the solutions

$$
V_{\alpha, j}=\Lambda_{\alpha}^{j} V_{\alpha},
$$

where $V_{0, j}$ escapes most rapidly from the origin, 0 .

The unstable curve, depicted in Figure 1, is defined by the solutions to (1.6), $X_{j}$, which project onto the most unstable mode as $j \rightarrow-\infty$. That is, we require

$$
X_{j} \propto \Lambda^{j} V_{0}, \quad \text { as } j \rightarrow-\infty .
$$

Note that a general solution to (1.6) contains modes other than $V_{0}$ as $j \rightarrow-\infty$. It therefore oscillates and tends to spiral around. In the limit as $\varepsilon \rightarrow 0^{+}$, the period of this oscillation is $q$. 
Now we define the parameterized unstable curve, $U(t)$. Since the unstable curve is invariant, $U(t)$ must obey $G(U(t))=U(\beta(t))$ for some monotonic function $\beta(t)$. Moreover, the conditions on the unstable curve at 0 imply that we must choose $U(0)=$ $0, U^{\prime}(0)=a V_{0}$, and $\beta^{\prime}(0)=\Lambda$. To specify the parameterized curve uniquely, we choose $\beta(t)=\Lambda t$ and $a=1$. In Appendix A, we prove Proposition 1, which states the existence and uniqueness of this curve.

Fontich and Simón ${ }^{22}$ proved the same result for the families of two-dimensional mappings that they have considered. Their proof utilizes the Birkhoff normal form theorem, whereas the proof in Appendix A makes use of the Banach's contraction mapping theorem.

Note that Proposition 1 implies that there exists a unique entire scalar function $u(t)$ such that

$$
U(t)=\left(u(t), u(\Lambda t), \ldots, u\left(\Lambda^{q-1} t\right)\right)
$$

and $u(t)$ is the solution of

$$
\begin{aligned}
u\left(\Lambda^{q} t\right) & =u(t)+\varepsilon g\left(u\left(\Lambda^{p} t\right)\right), \\
u(0) & =0, \quad \lim _{t \rightarrow 0} \frac{u(t)}{t}=1 .
\end{aligned}
$$

An orbit belonging to the unstable curve is determined by $t_{0}$, an initial value of the parameter $t$

$$
x_{j}=u\left(t_{j}\right), \quad t_{j}=\Lambda^{j} t_{0} \quad-\infty<j<\infty .
$$

Exactly the same approach can be used to define the stable curve $S(r)$. This stable curve is an invariant curve, which contains all the points that flow most rapidly into the fixed point at 1 . In analogy to (2.1), the linearization of (1.8) about 1 is

$$
W_{j+1}=D G^{-1}(1) W_{j}=\left(w_{j+q-1}-\varepsilon g^{\prime}(1) w_{j+p}, w_{j}, w_{j+1}, \ldots, w_{j+q-2}\right) \text {, }
$$

and the characteristic equation of $D G^{-1}(1)$ is

$$
\Gamma_{\alpha}^{q}=1-\varepsilon g^{\prime}(1) \Gamma_{\alpha}^{q-p},
$$

so that for small $\varepsilon$ we can write the solutions to $(2.12)$ as

$$
\Gamma_{\alpha}=\Omega^{-\alpha}\left[1-\frac{\varepsilon g^{\prime}(1) \Omega^{-\alpha(q-p)}}{q}\right]+O\left(\varepsilon^{2}\right)
$$

The eigenvector, $W_{\alpha}$, of the root $\Gamma_{\alpha}$ is

$$
W_{\alpha}=\left(1, \Gamma_{\alpha}^{-1}, \ldots, \Gamma_{\alpha}^{-(q-1)}\right),
$$

and the general solution to (2.11) is a linear combination of the solutions

$$
W_{\alpha, j}=\Gamma_{\alpha}^{-j} W_{\alpha} .
$$

The root with the largest magnitude is $\Gamma_{0}$, which we also denote as $\Gamma$. The parameterized stable curve, $S(r)$, is defined uniquely as stated in Proposition 1. 
As in the case for $U(t)$, it follows that there exists a unique entire function $s(r)$ such that

$$
S(r)=\left(s(r), s(r / \Gamma), \ldots, s\left(r / \Gamma^{q-1}\right)\right)
$$

and

$$
\begin{aligned}
s\left(\Gamma^{q} r\right) & =s(r)-\varepsilon g\left(s\left(r \Gamma^{q-p}\right)\right), \\
s(0) & =1, \quad \lim _{r \rightarrow 0} \frac{s(r)-s(0)}{r}=-1 .
\end{aligned}
$$

Orbits belonging to the stable curve are determined by $r_{0}$, the initial value of the parameter $r$

$$
x_{j}=s\left(r_{j}\right), \quad r_{j}=r_{0} / \Gamma^{j} .
$$

\section{B. Stable Manifold Theorem}

The stable and unstable curves can form, for example, the intricate structures shown in Figure 1. Nevertheless, according to Proposition 1, for any fixed $\varepsilon>0$, there exists a unique parameterization such that $U(t)$ and $S(r)$ are entire functions of $t$ and $r$ respectively and satisfy boundary condition $(1.12 b)$. Conversely, for fixed $t$ and $r$ there exists a nonempty interval $\left(0, \varepsilon_{\max }\right)$ such that $U(t)$ and $S(r)$ are each analytic in $\varepsilon$ for all $\varepsilon$ in the interior of this interval (but not at $\varepsilon=0$ ). Note that a simple generalization of the stable manifold theorem implies that the stable and unstable curves are unique and $C^{\infty}$, but does not imply anything regarding their analyticity or their parameterization. The work of Fontich and Simón ${ }^{22}$ is, to our knowledge, the first to address this issue.

\section{The Limit $\varepsilon \rightarrow 0^{+}$}

As discussed in the introduction, we are interested in studying the limit $\varepsilon \rightarrow 0^{+}$. For any $\varepsilon>0$, the fixed points are hyperbolic (though the hyperbolicity is nonuniform in $\varepsilon$ ). One can get a formal, but essentially correct, description of the limit $\varepsilon \rightarrow$ $0^{+}, j \varepsilon=O(1)$ by analyzing (2.9) (where $j$ counts the number of iterations of (1.6)). Consider that $u$ is a function of $t$ and $\varepsilon, u=u(t, \varepsilon)$, and write (2.9) as

$$
\frac{u\left(\Lambda^{q} t, \varepsilon\right)-u(t, \varepsilon)}{\varepsilon}=g\left(u\left(\Lambda^{p} t, \varepsilon\right)\right)
$$

where, from (2.2),

$$
\Lambda=1+\frac{\varepsilon g^{\prime}(0)}{q}+\frac{\varepsilon^{2} g^{\prime}(0)^{2}}{q^{2}}\left(p-\frac{q}{2}+\frac{1}{2}\right)+O\left(\varepsilon^{3}\right) .
$$

So long as $u(t, \varepsilon)$ is nonsingular in its variables, one can take the limit $\varepsilon \rightarrow 0^{+}$in 
(2.18) and find

$$
t \frac{d u_{0}(t)}{d t}=\frac{g\left(u_{0}(t)\right)}{g^{\prime}(0)}
$$

where

$$
u_{0}(t)=u(t, 0)
$$

The boundary condition on (2.19) is $u_{0}(0)=0$.

Table 3 lists solutions for this lowest-order solution $u_{0}(t)$ for the various $g$ 's described in Table 1 . In contrast to the exact $u(t)$, which is entire, each of these lowestorder solutions has singularities for finite values of $t$. We denote the singularities by $t_{s} \equiv\left|t_{s}\right| e^{i \phi_{s}}$. The positions of the singularities with the smallest phases, $\left|\phi_{s}\right|$, are shown in Table 3.

These singularities do not lie on the positive real axis. In fact, as $t$ gets large, each of these $u_{0}(t)$ approaches 1 . Therefore, in this continuum approximation our mapping has a heteroclinic connection between the fixed points at $\mathbf{0}$ and at $\mathbf{1}$.

An equivalent analysis gives the behavior of

$$
s_{0}(r)=\left.s(r, \varepsilon)\right|_{\varepsilon=0}
$$

From (2.16), we find

$$
r \frac{d s_{0}(r)}{d r}=\frac{g\left(s_{0}(r)\right)}{g^{\prime}(1)}
$$

with $s_{0}(0)=1$. The solutions of $(2.22)$ and $(2.19)$ are connected by the statement that they are identical except for a trivial change of parameterization

$$
u_{0}(t)=s_{0}(r(t))
$$

with

$$
r(t)=b_{0} t^{g^{\prime}(1) / g^{\prime}(0)}
$$

For completeness we list the values of $b_{0}$ in Table 3.

The continuum approximation is a quantitatively correct description of $u(t, \varepsilon)$ and $s(r, \varepsilon)$ for fixed and not-too-large real values of $t$ and $r$. However, it fails in at least two limits:

i. near the singularities of $u_{0}(t)$ and $s_{0}(r)$ in $t$ and $r$;

ii. as $t$ and $r$ go to infinity.

In the latter case, there is the possibility that $u$ and $s$ can accumulate small oscillatory terms (like the possible period- $q$ oscillations in $x_{j}$ which were already noted) and that these oscillations can ruin the heteroclinic connections (see Figure 1). In fact, we shall see that if $t$ is real, positive, and of order $1, u\left(\Lambda^{j} t\right)$ can be dominated by oscillatory terms when $j \sim 1 / \varepsilon^{2}$. 


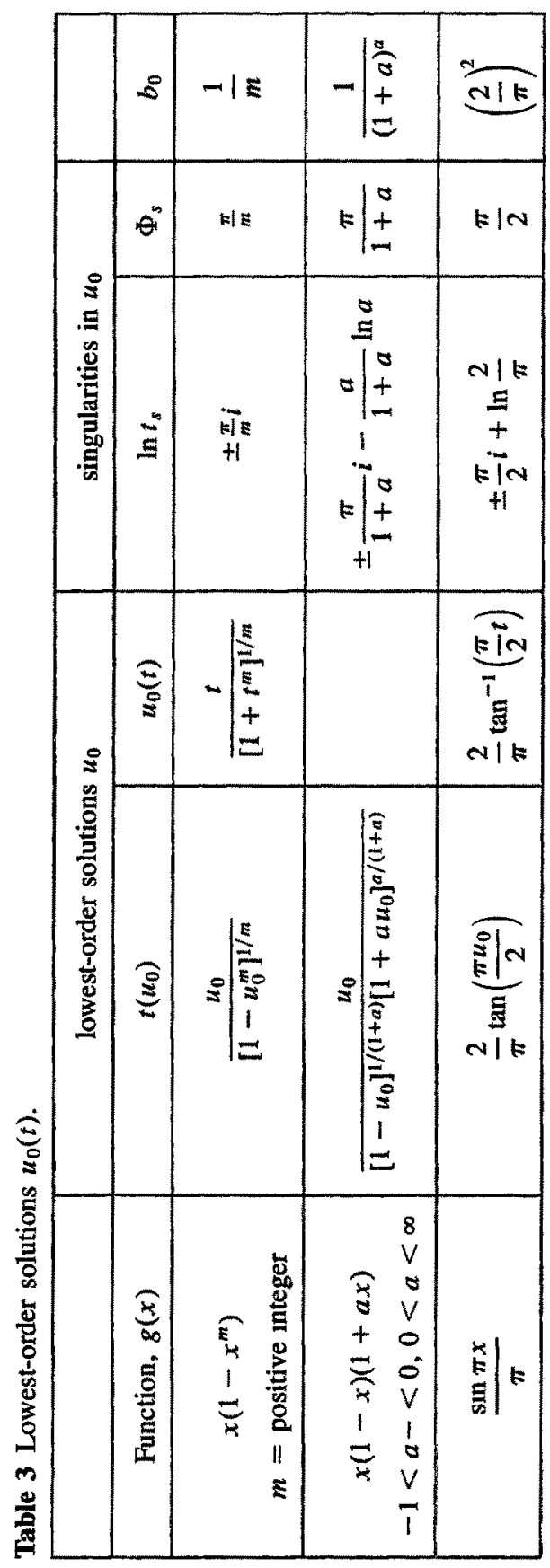




\section{Possible Behavior of the Curves}

Since $u_{0}(t)$ does correctly represent the behavior of $u(t)$ when $\varepsilon$ is sufficiently small and $t$ is large but not too large (see Appendix D), there will be a region of $t$ for which $U(t)$ is very close to 1 . In this region, we can apply the linear stability analysis about the fixed point 1; using (2.14), we find

$$
U(t)=U\left(\Lambda^{j} t_{0}\right)=1-\sum_{\alpha} \tilde{b}_{\alpha}\left(t_{0}\right) W_{\alpha} \Gamma_{\alpha}^{-j}+Q(t)
$$

where $Q(t)$ stands for the quadratic terms. Rewriting this equation in terms of $t$, and neglecting the small quadratic terms, we find that $U(t)$ is of the form

$$
U(t) \approx 1-\sum_{\alpha} \frac{b_{\alpha}(t) W_{\alpha}}{t^{\ln \Gamma_{\alpha} / \ln \Lambda}}
$$

Here, $b_{\alpha}$ may depend upon $t$ because different $t$-values may represent different orbits. However, if one stays on one orbit, an iteration gives $t \rightarrow \Lambda t$. Therefore, the $b_{\alpha}$ 's are periodic in $\ln t / \ln \Lambda$

$$
b_{\alpha}(\Lambda t)=b_{\alpha}(t)
$$

As we shall see, for small $\varepsilon$ and $\alpha \neq 0$, the $b_{\alpha}$ 's are extremely small; hence it is justified to neglect the quadratic terms in (2.25).

Several different behaviors are possible. The first is the simplest.

1. $b_{\alpha}(t)$ vanishes for $\alpha \neq 0$. Then all points on $U(t)$ also lie on $S(r)$ as $t \rightarrow \infty$. Since $S(r)$ is uniquely defined by the $r \rightarrow 0$ limit, the stable and unstable curves must be identical, and their parameterizations satisfy

$$
U(t)=S\left(b_{0}(t) t^{-\ln \Gamma / \ln \Lambda}\right) .
$$

Thus in this first case, there is a heteroclinic connection between the fixed points at $\mathbf{0}$ and 1.

2. Some of the $b_{\alpha}$ 's for $\alpha \neq 0$ are nonzero, but $b_{\alpha}$ vanishes for all the $\alpha$ 's which have $\left|\Gamma_{\alpha}\right|<1$. In this case, the unstable curve is contained in the stable manifold of 1 ; hence,

$$
\lim _{t \rightarrow \infty} U(t)=1
$$

and there is a heteroclinic connection, but now the two curves are different. This case cannot arise for $q=2$ (where $\Gamma_{1}<1$ ) or $q=3$ (where $\left|\Gamma_{ \pm 1}\right|<1$ ), but can arise for $q \geq 4$.

3. Some of the $b_{\alpha}$ 's for $\alpha \neq 0$ and $\left|\Gamma_{\alpha}\right|<1$ are nonvanishing. Then, (2.25) implies that as $t$ gets so large that the $\alpha \neq 0$ terms in (2.25) become large (yet the quadratic terms are still small), $U(t)$ moves away from 1 . Therefore, the heteroclinicity condition (2.28) is likely to fail. (It is possible but unlikely that for still larger $t$ 's $U(t)$ might approach 1.) There can still be a discrete set of $t^{*}$ with $1 \leq t^{*} \leq \Lambda$ 
for which

$$
b_{\alpha}\left(t^{*}\right)=0, \quad \text { for all } \alpha \neq 0
$$

In this case the stable and unstable manifold will cross an infinite number of times. Fontich and Simón ${ }^{22}$ show that for $q=2$, case 3 holds and that there is a $t^{*}$ for which $b_{1}\left(t^{*}\right)=0$. In fact, the planar geometry of $q=2$, together with the areapreserving property of the map, demands that if the stable and unstable curves are not identical they must cross. Then, if they cross once, of course they will cross an infinite number of times.

Our numerical evidence suggests that case 3 is generic. Moreover, it suggests that generically, unless $q=2$ or $q=4$ and $p=1$, crossings do not occur (see Section IV.C). Nonetheless, the other cases are possible.

To see how case 1 might arise, specify $u(t)$ so that $u(0)=0, u(\infty)=1$ and such that $u(t)$ is analytic in $t$ near $t=0$ and analytic in an inverse power of $t$ near $t=\infty$.

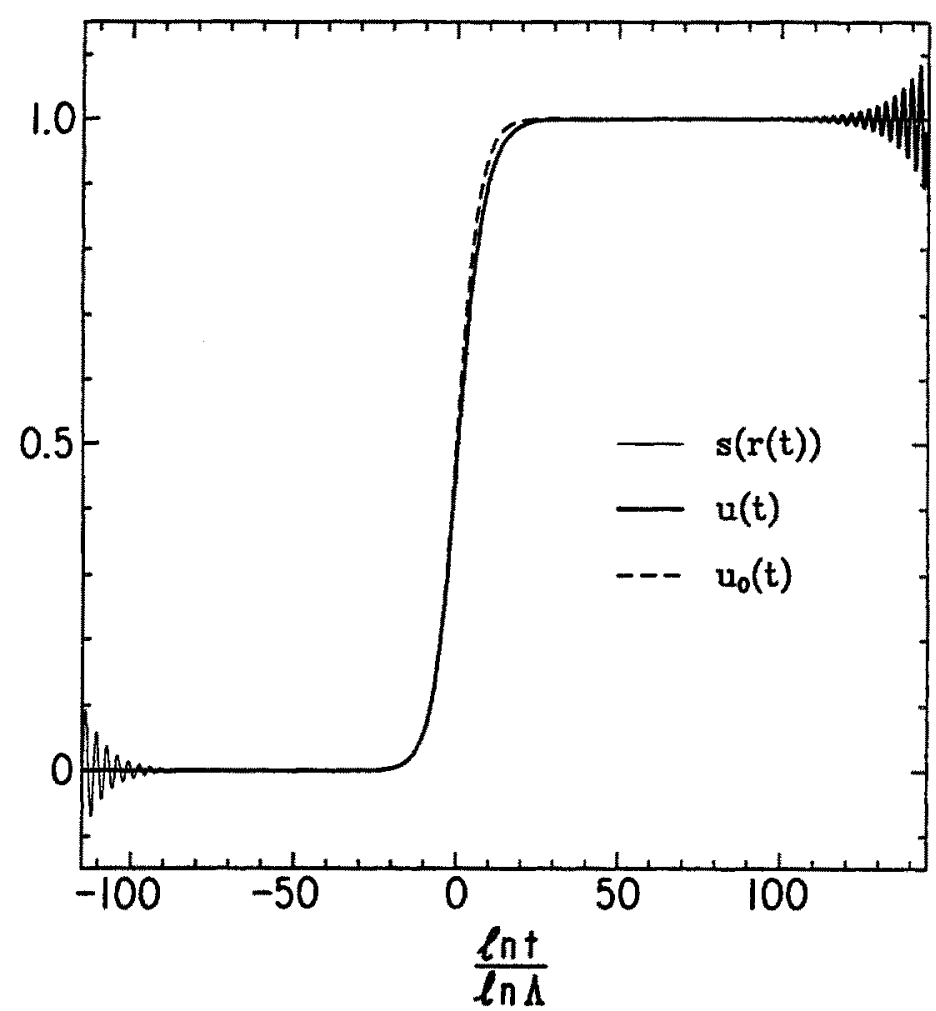

Fig. 3. The one-dimensional projection of the stable and unstable curves and their singular limit as $\varepsilon \rightarrow 0$. We plot $s(r(t)), u(t)$, and $u_{0}(t)$ as a function of $\ln t / \ln \Lambda$ for $q=3, p=2$, and $g(x)=x(1-x)$ with $\varepsilon=0.75$. Notice that $u(t)$ and $s(r(t))$ are indistinguishable at this scale, except for the oscillations near the end points 0 and 1 . 
For example, use the $m=1$ case of line 1 in Table 3

$$
u(t)=\frac{t}{1+t}
$$

Now ask whether there is any $g(x)$ for which this $u(t)$ serves as a solution to (2.9). If such a solution exists, it defines a heteroclinic connection in which the two curves are identical.

Simple algebra solves $(2.9 a)$ for $g$, giving

$$
g(x)=\frac{x(1-x)}{\left[1+\left(\Lambda^{q-p}-1\right) x\right]\left[1+\left(\Lambda^{-p}-1\right) x\right]}
$$

which works when $\varepsilon$ is defined by

$$
\varepsilon=\frac{\Lambda^{q}-1}{\Lambda^{p}}
$$

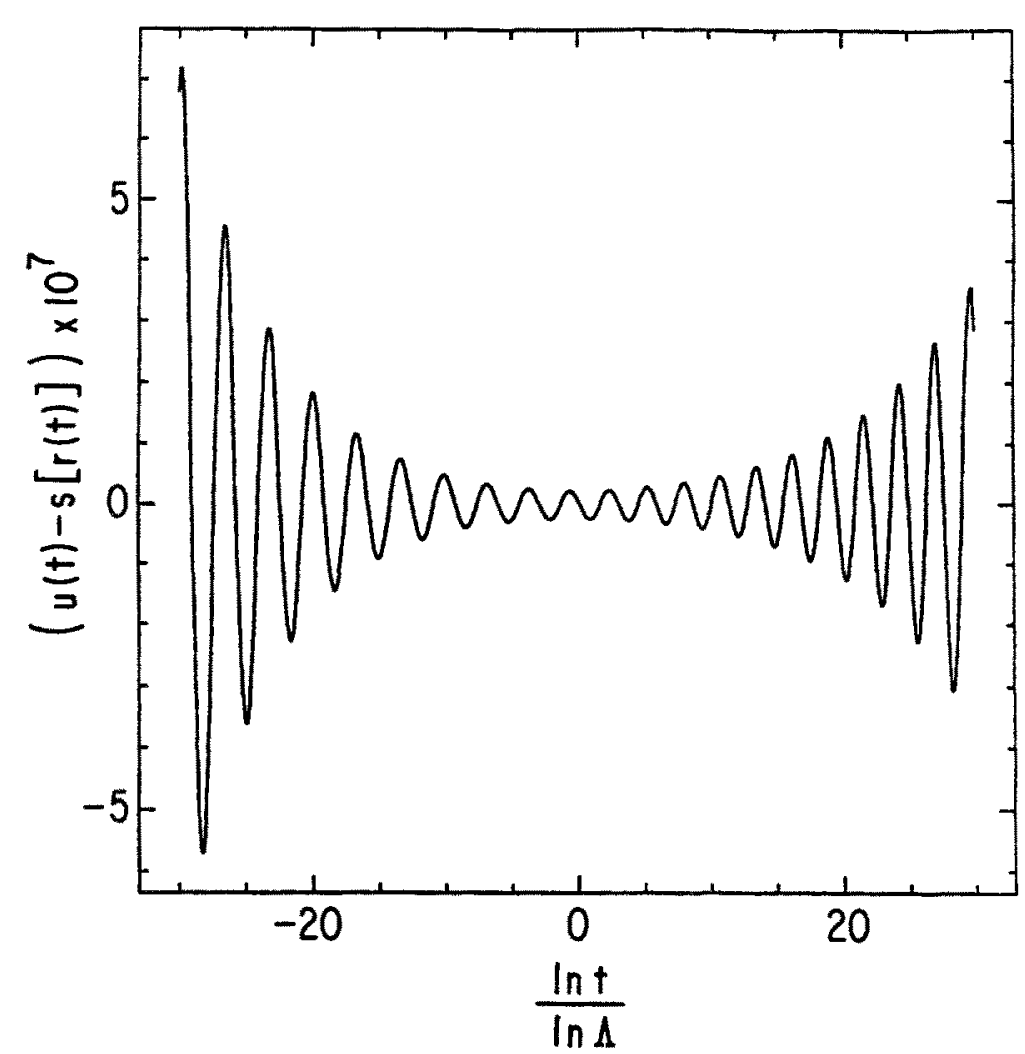

Fig. 4. The one-dimensional difference between the unstable and stable curves. We plot $u(t)-s(r(t))$ versus $\ln t / \ln \Lambda$ for a small central region in Figure 3. The period of the oscillations in the figure is approximately $q=3$ and corresponds to the period of the linear modes $v_{ \pm 1}(t)$; see (3.6) and (3.8). 
Therefore, case 1 can hold. However, notice that $g(x)$ in $(2.30 b)$ is not an entire function of $x$.

In Appendix A we prove that when $g(x)$ is entire and $\varepsilon>\tilde{\varepsilon}(g, q, p)$, the heteroclinicity condition (2.28) fails; hence only case 3 is possible. For example, if $g(x)=x(1-x), q=3, p=2$, and $\varepsilon>\frac{63}{16}$ then the stable and unstable curves cannot coincide. We note that the large $\varepsilon$ behavior may be unrelated to the $\varepsilon \rightarrow 0^{+}$ limit considered in this paper as the method used in the proof does not seem to be continuable to small $\varepsilon$ values. Nevertheless, we include the proof in Appendix A for its elegance.

\section{E. Numerical Data}

In this section, we present numerical evidence supporting the qualitative assertions made above. The case we picked is $q=3, p=2$, and $g(x)=x(1-x)$. We calculate $u(t)$ and $s(r)$ by the methods described in Appendix C. Figure 3 is a plot of $u(t), s(r)$, and $u_{0}(t)$ for $\varepsilon=0.75$. The $r$ and $t$ scales are taken so that (see 2.24)

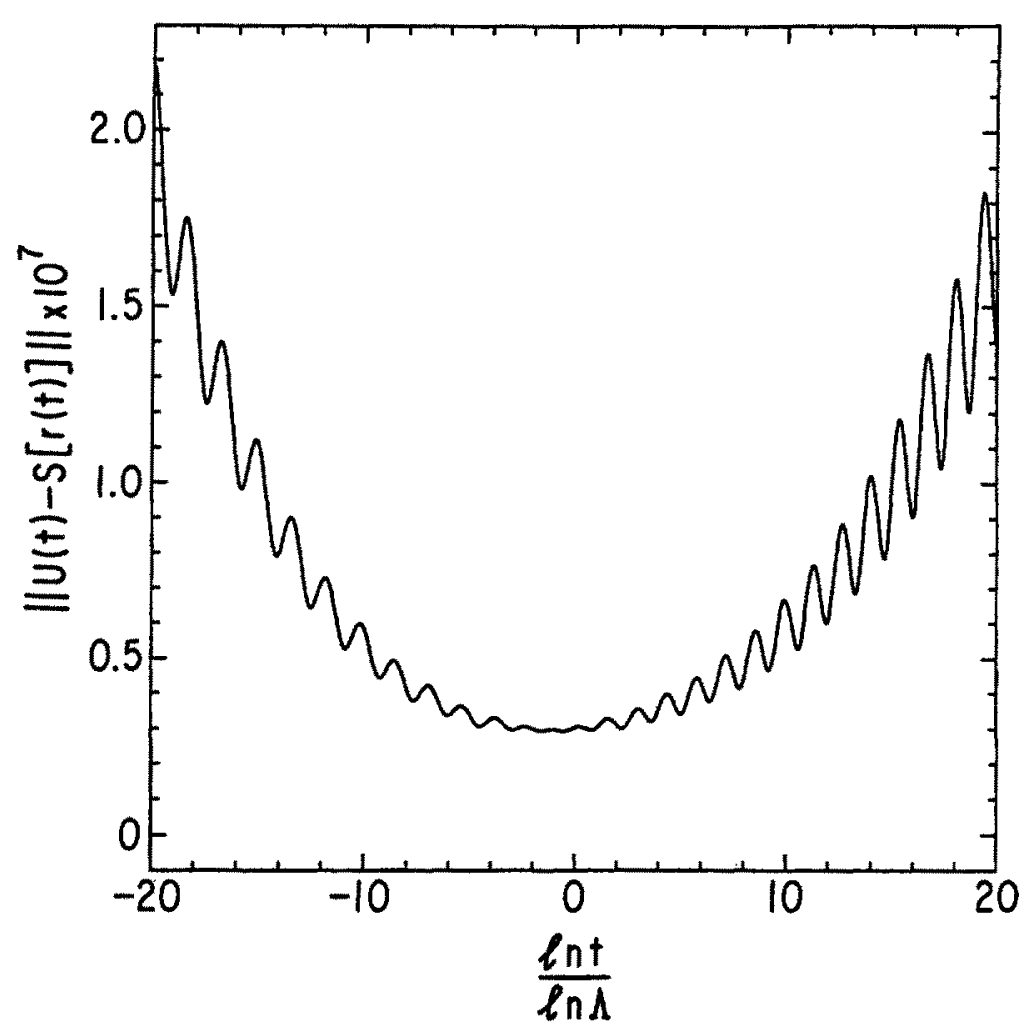

Fig. 5. The distance between the stable and unstable curves. Here we plot $\|S(r(t))-U(t)\|$ as a function of $\ln t / \ln \Lambda$ for the same case as in Figure 3. The minimal distance occurs when $t \sim 1$, where the linear modes $V_{ \pm_{1}}(t)$ and $W_{ \pm 1}(r(t))$ have a magnitude of order 1 . 


$$
r(t)=\frac{b_{0}}{t^{\ln \Gamma / \ln \Lambda}}
$$

and $b_{0}$ is chosen to minimize the distance between $U\left(t_{1}\right)$ and $S\left(r\left(t_{1}\right)\right)$, where $t_{1}$ is defined by

$$
u\left(t_{1}\right)=u_{0}(1)
$$

thus, $t_{1}=1+O(\varepsilon)$.

Notice that $u_{0}(t)$ is only roughly similar to $u(t)$ but that $u(t)$ and $s(r(t))$ agree very

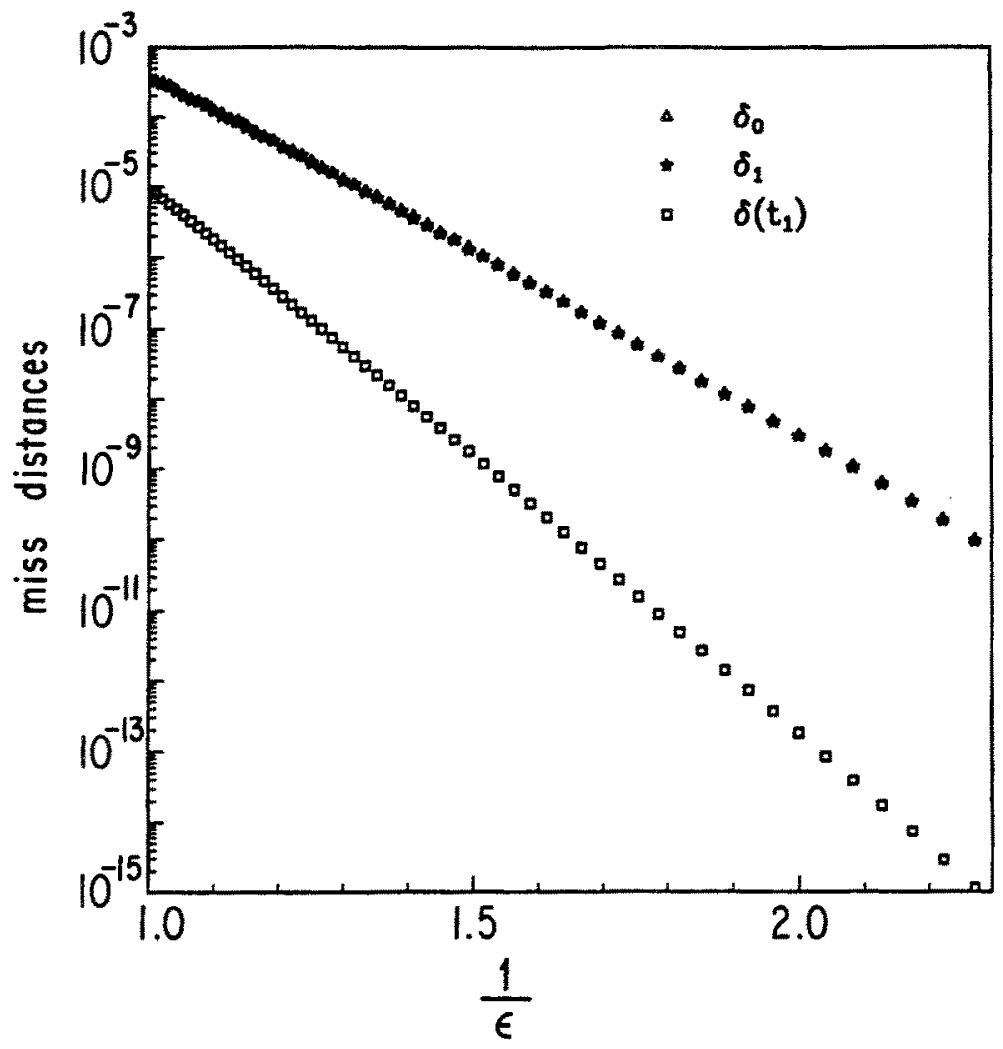

Fig. 6. A graph of several "miss distances" as a function of $1 / \varepsilon$. The miss distances $\delta_{0}$ and $\delta_{1}$ are, respectively, the minimal distance of the stable curve from the fixed point 0 and the minimal distance of the stable curve from the fixed point 1 . The miss distance $\delta\left(t_{1}\right)$ is the distance between the stable and unstable curves at the $t$ value $t_{1}$; see (2.32). The case shown is $q=$ $3, p=2$, and $g(x)=x(1-x)$. We see that all these miss distances exhibit an exponential dependence on $1 / \varepsilon$, that the slopes of $\ln \delta_{0}$ and $\ln \delta_{1}$ are indistinguishable, and that their slope is smaller in magnitude than the slope of $\ln \delta\left(t_{1}\right)$. These results are in accordance with (1.9) and (1.10). The exact value of the coefficient of $1 / \varepsilon$ in the exponent cannot be extracted accurately from this figure since in this range of $\varepsilon$ values the power-law correction in $\varepsilon$ is significant. 
closely in the central region of the plot. As expected, $s(r)$ and $u(t)$ each oscillates near one end of the graph.

Next, in Figure 4, we plot $u(t)-s(r(t))$ for a small central region of Figure 3. Notice the tiny (but crucial) oscillation which appears to have a period 3 in $\ln t / \ln \Lambda$. This oscillation produces the separation between the $U(t)$ and $S(r(t))$ curves depicted in Figure 5.

Using results like those depicted in Figure 5, we calculate the separation, $\delta$, of the two curves, $S(r(t))$ and $U(t)$, at $t_{1}$. This separation is plotted as a function of $1 / \varepsilon$ in Figure 6. Notice the apparent straight-line behavior on this log-linear plot, which seems to agree with the leading term in (1.9). We shall make a more careful analysis of this fit in Section IV. Note that in Figure 6, $\varepsilon \geq 0.434$. The smallness of $\delta(t)$ for real $t$ inhibits a numerical calculation for smaller values of $\varepsilon$. We obtain numerical support to (1.9) in the limit $\varepsilon \rightarrow 0^{+}$by calculating $\delta(t)$ for complex $t$ (see Section IV). There, we can carry out calculations for values of $\varepsilon$ as small as 0.005 .

In Figures 7a and $7 \mathrm{~b}$, we show plots of the distance of $U(t)$ and $S(r)$ from the fixed points at 1 and 0 respectively for $\varepsilon=0.75$. These figures also define the minimum

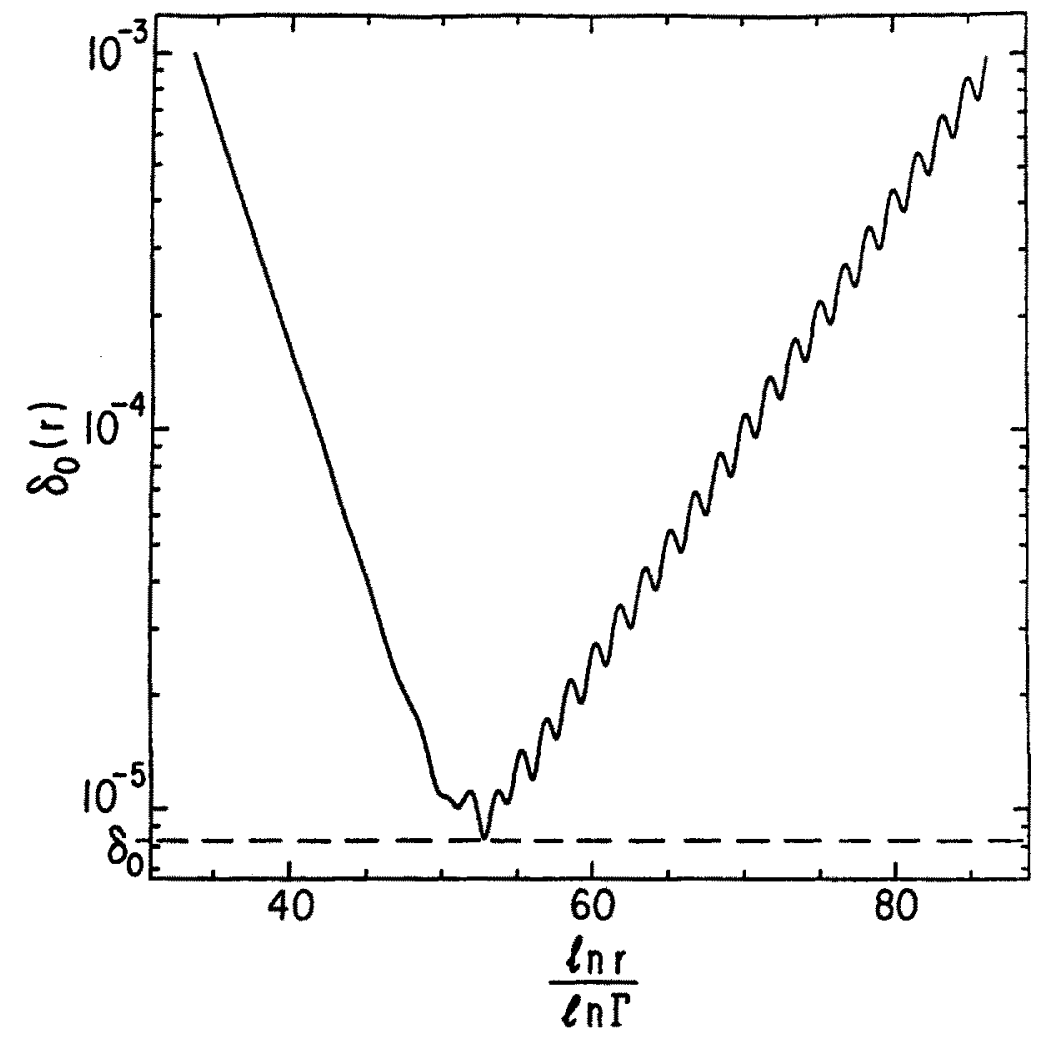

Fig. 7a. The calculation of the miss distances from the fixed points. In Figures $7 \mathrm{a}(7 \mathrm{~b})$ we plot $\delta_{0}(r)\left(\delta_{1}(t)\right)$ as a function of $\ln r / \ln \Gamma(\ln t / \ln \Lambda)$ for the same case as in Figure 3 . The existence of a global minimum $\delta_{0}\left(\delta_{1}\right)$ is evident. 
separations $\delta_{0}$ and $\delta_{1}$. In Figure 6 we also plot $\delta_{0}$ and $\delta_{1}$ versus $1 / \varepsilon$. The quantities $\ln \delta_{0}$ and $\ln \delta_{1}$ also seem to show a linear behavior in $1 / \varepsilon$, but with a slope smaller than that of $\ln \delta$.

\section{Linear Deviation Analysis}

\section{A. The Linear Deviations}

The functions $u(t)$ and $s(r(t))$ described above obey exactly the same equation, but with different boundary conditions. Let $w(t)$ stand for either $u(t)$ or $s(r(t))$ when the parameterization is chosen so that an increase of $t$ by $\Lambda$ corresponds to a decrease of $r$ by $\Gamma$ (see (1.12))

$$
r(\Lambda t)=\frac{1}{\Gamma} r(t)
$$

Then $w(t)$ obeys (see (2.9))

$$
w\left(\Lambda^{q} t\right)=w(t)+\varepsilon g\left(w\left(\Lambda^{p} t\right)\right) .
$$

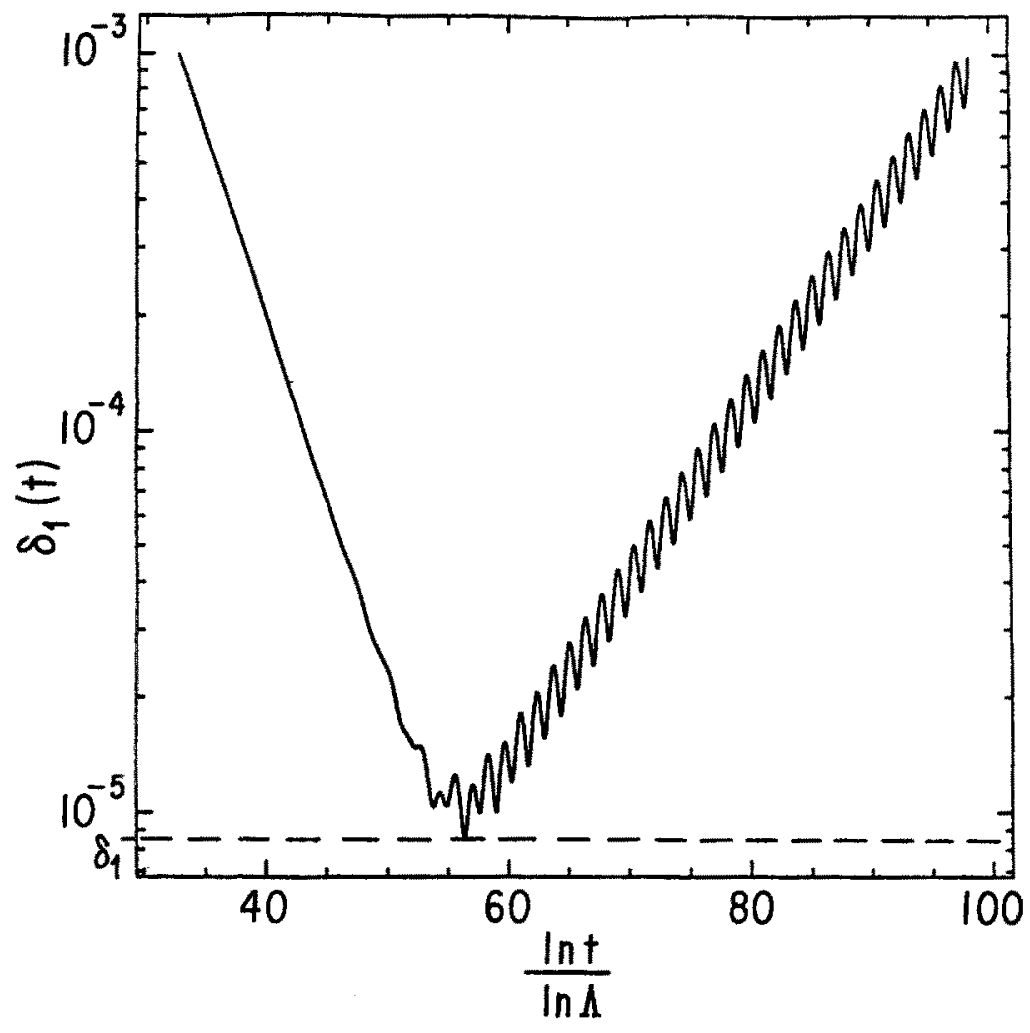

Fig. 7 b. (continued) 
Now consider a $w(t)$ which differs only infinitesimally from $u(t)$

$$
w(t)=u(t)+v(t)
$$

with $v(t)$ being very small. Then (3.2) implies

$$
v\left(\Lambda^{q} t\right)=v(t)+\varepsilon g^{\prime}\left(u\left(\Lambda^{p} t\right)\right) v\left(\Lambda^{p} t\right)
$$

Naturally, we are thinking about the situation in which $v(t)$ represents the very small difference between the stable and unstable curves.

Exactly the same logic that led to $(2.25)$ implies that if $t \ll 1$, then. $v(t)$ must be of the form

$$
v(t)=\sum_{\alpha} c_{\alpha}(t) v_{\alpha}(t)
$$

where in the small $t$ limit $v_{\alpha}$ goes to $t^{\chi_{\alpha}}$, with

$$
\chi_{\alpha}=\frac{\ln \Lambda_{\alpha}}{\ln \Lambda}
$$

As before, the expansion coefficients must satisfy a periodicity condition

$$
c_{\alpha}(\Lambda t)=c_{\alpha}(t)
$$

but they are otherwise arbitrary.

In exactly the same way as we defined $u(t)$, using its linear behavior as $t \rightarrow 0$, we now define $v_{\alpha}(t)$ for $0<t<\infty$. Demand that $v_{\alpha}(t)$ be a solution to (3.4). To specify the behavior as $t \rightarrow 0$, require that $\tilde{v}_{\alpha}(t)$ defined by

$$
v_{\alpha}(t)=t^{x_{\alpha}} \tilde{v}_{\alpha}(t)
$$

be analytic at $t=0$ and go to 1 as $t \rightarrow 0$

$$
\tilde{v}_{\alpha}(0)=1 \text {. }
$$

These requirements define $v_{\alpha}$ and $\tilde{v}_{\alpha}$ uniquely. Substituting (3.8) into (3.4), we see that the latter function obeys

$$
\Lambda_{\alpha}^{q} \tilde{v}_{\alpha}\left(\Lambda^{q} t\right)=\tilde{v}_{\alpha}(t)+\varepsilon g^{\prime}\left(u\left(\Lambda^{p} t\right)\right) \Lambda_{\alpha}^{p} \tilde{v}_{\alpha}\left(\Lambda^{p} t\right) .
$$

If $g(x)$ is an entire function of $x$, then $\tilde{v}_{\alpha}(t)$ is, for sufficiently small $\varepsilon>0$, entire in $t$ and analytic in $\varepsilon$.

Now that $v_{\alpha}(t)$ is defined, we can state the main result of this section. Let

$$
\Delta(t)=S(r(t))-U(t)
$$

We write (3.1) as

$$
r(t)=\frac{b_{0}(t)}{t^{\ln \Gamma / \ln \Lambda}}
$$


with

$$
b_{0}(\Lambda t)=b_{0}(t)
$$

We assert that

$$
\Delta(t)=\sum_{\alpha} c_{\alpha}(t) V_{\alpha}(t)+O\left(\delta^{2}\right)
$$

where $\delta=\delta\left(t_{1}\right)=\left\|\Delta\left(t_{1}\right)\right\|$. Our job, then, has been reduced to the calculation of the coefficients $c_{\alpha}(t)$ and the parameterization $b_{0}(t)$. As long as $\delta \ll 1$, this calculation will give an excellent estimate for $\Delta(t)$. It is important to realize that the error term in (3.12) is the outcome of the linearization about $U(t)$, which does not involve any expansion in $\varepsilon$. Therefore, its magnitude is proportional to the quadratic terms in the splitting distance. This is a key point in our analysis; given that the splitting distance is exponentially small, the next-order term is indeed negligible. This is to be contrasted with the usual situation in which the leading-order term in a regular expansion in $\varepsilon$ is exponentially small and the higher-order terms must be shown to be exponentially small as well. For finite, real intervals of $t$, one can prove quite easily that (3.12) is valid. Therefore, we shall ignore the error term for now, and identify $V(t)=\sum c_{\alpha} V_{\alpha}$ with the vector of the splitting distance $\Delta(t)$.

An important property of the $c_{\alpha}$ 's is that they are periodic functions of $\ln t$ with period $\ln \Lambda$ and hence can be expressed as a Fourier series

$$
c_{\alpha}(t)=\sum_{n} c_{\alpha, n} \exp \left(\frac{2 \pi i n \ln t}{\ln \Lambda}\right)
$$

The condition that $s$ and $u$ be real gives conditions on the complex conjugate of $c_{\alpha, n}$, namely,

$$
c_{\alpha, n}^{*}= \begin{cases}c_{-\alpha,-n}, & \text { for } \alpha \neq q / 2, \\ c_{\alpha,-1-n}, & \text { for } \alpha=q / 2 .\end{cases}
$$

The coefficient $c_{0}(t)$ measures the distance between $s(r(t))$ and $u(t)$ in the tangential direction of $u(t)$. Indeed, $\tilde{v}_{0}(t)$ and $d u(t) / d t$ obey precisely the same equation (differentiate (2.9a) and compare with (3.9b)). Hence, (3.12) can be written as

$$
\begin{aligned}
s(r(t)) & =u(t)+c_{0}(t) t \frac{d u}{d t}+\sum_{\alpha \neq 0} c_{\alpha}(t) v_{\alpha}(t) \\
& \approx u\left(t\left(1+c_{0}(t)\right)\right)+\sum_{\alpha \neq 0} c_{\alpha}(t) v_{\alpha}(t) .
\end{aligned}
$$

The above equation demonstrates that the only effect of $c_{0}(t)$ is to change the parameterization of the stable curve. Therefore, we define the parameterization $r(t)$ (via the function $b_{0}(t)$ ) so that

$$
c_{0}(t)=0 .
$$




\section{B. Small $\varepsilon-$ Results}

To understand the behavior of (3.12) for small $\varepsilon$, it is instructive to look at the form of $v_{\alpha}(t)$ for small $t$. Equation (3.8) expresses $v_{\alpha}(t)$ as a product of two factors. The first is $t^{\chi_{\alpha}}$, and for small $\varepsilon,(3.6),(2.4)$, and $(2.18 b)$ give

$$
\chi_{\alpha}=\frac{2 \pi i \alpha}{\varepsilon g^{\prime}(0)}+\Omega^{\alpha p}+2 \pi i \alpha\left(\frac{1}{2}-\frac{p}{q}\right)
$$

The term in $\varepsilon^{-1}$ gives $v_{\alpha}(t)$ an extreme sensitivity to the phase of $t$. If $t=|t| e^{i \phi}$, then the leading term in $\left|v_{\alpha}(t)\right|$ is

$$
\left|v_{\alpha}(t)\right| \sim e^{-2 \pi \alpha \phi / \varepsilon} .
$$

This behavior will, in the end, give the $\varepsilon^{-1}$ term in $\ln \delta$.

On the other hand, the remaining factor in $v_{\alpha}(t)$, namely, $\tilde{v}_{\alpha}(t)$, is not very sensitive to $\varepsilon$. If we fix $t$ and take $\varepsilon$ to zero in $(3.9 b)$, the terms of order $\varepsilon$ in that equation give

$$
\Omega^{\alpha p} \tilde{v}_{\alpha}(t)+t \frac{d}{d t} \tilde{v}_{\alpha}(t)=\frac{g^{\prime}(u(t))}{g^{\prime}(0)} \Omega^{\alpha p} \tilde{v}_{\alpha}(t)
$$

Since $u(t) \rightarrow u_{0}(t)$ as $\varepsilon \rightarrow 0^{+}$, we can use (2.19) to find

$$
\tilde{v}_{\alpha}(t)=\left(\frac{g\left(u_{0}(t)\right)}{g^{\prime}(0) t}\right)^{\Omega^{\alpha p}} \text {. }
$$

Equation (3.16) holds in the limit $\varepsilon \rightarrow 0^{+},|t|$ of order unity or smaller. It is also required that $t$ be far from the singularities of $u_{0}(t)$. In this limit, $\tilde{v}_{\alpha}(t)$ is not very large, and it is nonsingular.

\section{Numerical Work}

Neglecting the quadratic terms, (3.12) can be translated into an equation obeyed by the phase space functions $U(t)$ and $S(r)$, namely,

$$
S(r(t))-U(t)=\sum_{\alpha} c_{\alpha}(t) V_{\alpha}(t)
$$

where the phase space version of $v_{\alpha}(t)$ is

$$
V_{\alpha}(t)=\left(v_{\alpha}(t), v_{\alpha}(\Lambda t), \ldots, v_{\alpha}\left(\Lambda^{q-1} t\right)\right)
$$

and $r(t)$ is given by

$$
r(t)=\frac{b_{0}(t)}{t^{\ln \Gamma / \ln \Lambda}}
$$

where $b_{0}(t)$ is chosen so that $c_{0}(t)=0$. The functions $U(t), S(r(t))$, and $V_{\alpha}(t)$ are computed directly from their definitions (see Section IV.E). Hence, all the quantities 
in (3.17) are known numerically except for the coefficients $c_{\alpha}(t)$. Since the vectors $V_{\alpha}(t)$ span the $q$-dimensional Euclidean space, $c_{\alpha}(t)$ can be computed directly from (3.17) by inverting the $q$-by- $q$ matrix of components of $V_{\alpha}(t)$.

The neglecting of the quadratic terms in (3.17) is justified if the right-hand side is small. A check on the correctness is that $c_{\alpha}(\Lambda t)=c_{\alpha}(t)$. Figure 8 is a plot of $\left|c_{ \pm 1}(t)\right|$ for $g(x)=x\left(1-x^{2}\right), q=5, p=2$, and $\varepsilon=0.5$. Notice that the $\left|c_{ \pm 1}\right|$ are small and not only periodic but apparently constant; the $t$-dependence shown in the figure is of the order of the quadratic terms $\left|c_{ \pm 1}\right|^{2}$, and this is consistent with (3.12) since, as we shall see in the next section, $\delta \sim\left|c_{ \pm 1}\right|$. We thus argue that, except for exponentially small terms, $\left|c_{-1}\right|$ is independent of $t$.

In all cases, we find that the leading term on the right-hand side of (3.17) is the one involving $c_{ \pm 1}(t)$. All the remaining $c_{\alpha}$ 's are much smaller. In these cases, except for $q=2, c_{ \pm 1}(t)$ is essentially independent of $t$. For $q=2, c_{1}(t)$ is the leading and only term, and it oscillates.

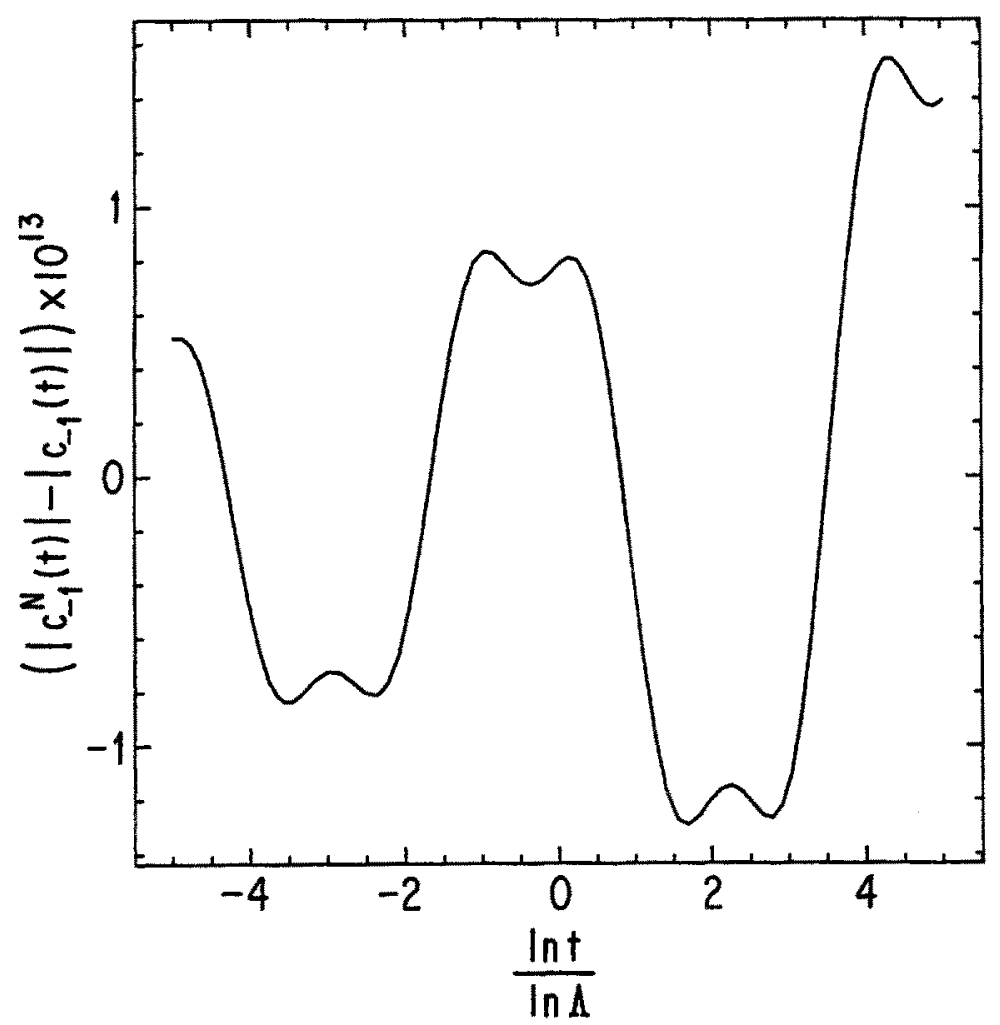

Fig. 8. The dependence of the numerical value of $\left|c_{ \pm 1}\right|$ on $t$. We plot $\left|c_{-1}^{N}(t)\right|-\left|c_{-1}\right|$ as a function of $\ln t / \ln \Lambda$ for the case $g(x)=x\left(1-x^{2}\right), q=$ 5 , and $p=2$ with $\varepsilon=0.5$. Here $c_{-1}^{N}(t)$ is the result of the numerical computation and $\left|c_{-1}\right|=2.89956 \times 10^{-7}$. The oscillations in $t$ are of order $\left|c_{-1}\right|^{2}$ and correspond to quadratic errors; see Appendix C. 


\section{Analysis in the Complex Plane}

Recall the procedure suggested by Kruskal and Segur ${ }^{19}$ for attacking singular perturbation problems:

1. Find solutions which fit the desired equations with all the freedom allowed by the boundary conditions. In our case, these are $U(t)$ and $S(r)$ with the addition of the small perturbation terms $c_{\alpha}(t) V_{\alpha}(t)$.

2. Fit the solutions to the appropriate boundary conditions at the appropriate endpoints. Here we get $U$ and $S$.

3. Find a place, usually not in the real domain, where the low-order solutions are singular. In this region, the small deviations will be exponentially enhanced. Use this region to match the solutions. In our case, the matching corresponds to the determination of the coefficients $c_{\alpha}(t)$.

4. Take the matched solutions and analytically continue them back into the physical domain. The correction terms will be correctly estimated even though they are exponentially small.

The pitfall of the above procedure is that one usually finds the leading-order behavior of the various quantities, and must verify that in the matching region, these quantities are still well approximated by the leading-order terms. In our case, we would like to match the left-hand side and the right-hand side of (3.12), and use the lowest-order solutions $u_{0}(t), s_{0}(t)$, and $\tilde{v}_{\alpha}(t)$ to estimate the order of magnitude of the terms in (3.12). To justify this procedure, we need to prove that the error term $O\left(\delta^{2}\right)$ in (3.12) is small, and that the expansions in $\varepsilon$ of $u, s$, and $\vec{v}_{\alpha}$ are not failing too badly in the matching region. In Appendix $D$, we formulate the above statements more precisely and outline the arguments for the smallness of the quadratic terms in a region bounded away from the singularity.

\section{A. Exponential Estimate of $\delta(t)$}

In our case, the low-order solutions are easily found. In fact, we exhibited the lowestorder solution for the case $g(x)=x(1-x)$ in Table 3. Here we look at the continuum limit of the recursion relation and find, for example, for $g(x)=x(1-x)$, a lowestorder solution

$$
\lim _{\varepsilon \rightarrow 0} u(t)=u_{0}(t)=\frac{t}{1+t}
$$

with $x_{j}=u\left(t_{j}\right)$ and to the lowest-order

$$
t_{j}=e^{j \varepsilon / q} t_{0}
$$

Clearly, the solution (4.1) has a singularity at $t=-1$.

The qualitative reason for this singularity is easily seen. The unstable curve near $t=0$ is defined by $x_{j}=\Lambda^{j} t_{0}$, with $t_{0}$ small. Imagine that $t_{0}$ is a negative real number. Then each $x_{j}$ is negative and real. Successive $\left|x_{j}\right|$ 's get larger and larger as $j$ 
increases. Since

$$
x_{j+q}=x_{j}+\varepsilon x_{j+p}\left(1-x_{j+p}\right),
$$

as $\left|x_{j}\right|$ increases the steps get larger and larger until, when $x_{j}$ gets to be of order $-1 / \varepsilon$, the steps between successive $x_{j}$ 's get to be as large as the $x_{j}$ 's themselves. Our continuum solution then becomes meaningless. Nonetheless, we can use the neighborhood of $x_{j} \sim-1 / \varepsilon$ as a fitting region for the different solutions. Similarly, we define the matching region for a general function $g$ to be the region in which the continuum limit begins to break down, namely, where $x_{j} \sim \varepsilon g\left(x_{j}\right)$.

Notice that we have here a singularity which arises in the low-order solution when the phase of $t$ approaches $\pm \pi$. Now let us examine the form of the $v_{\alpha}(t)$ in this region of $t$. It follows from (3.8) and (3.15) that if $\alpha>0(\alpha<0)$ the first factor in $v_{\alpha}(t)$, $t^{\chi_{\alpha}}$, gets exponentially large as the phase of $t$ approaches $-\pi(\pi)$. Our lowest-order estimate of $v_{\alpha}\left(t_{s}\right)$ is

$$
\left|v_{\alpha}\left(t_{s}\right)\right| \sim \exp \left[-\frac{2 \pi \alpha \phi_{s}}{\varepsilon g^{\prime}(0)}\right]
$$

In writing (4.3), we have neglected any factors which might arise from $\tilde{v}_{\alpha}\left(t_{s}\right)$. In fact, $\tilde{v}_{\alpha}(t)$ does get large near $t_{s}$, but, as we shall argue (see, however, the discussion at the beginning of Section IV), the growth is only algebraic in $\varepsilon^{-1}$ and hence may be neglected for this lowest-order analysis. Recall our basic very small parameter

$$
\xi \equiv \xi(0)=\exp \left[-\frac{2 \pi \phi_{s}}{\varepsilon g^{\prime}(0)}\right]
$$

and write the estimate (4.3) as

$$
\left|v_{\alpha}\left(t_{s}\right)\right| \sim \xi^{\alpha}
$$

Since $t_{s}^{*}$ is also a singular point of $u_{0}$, a similar calculation gives

$$
\left|v_{\alpha}\left(t_{s}^{*}\right)\right| \sim \xi^{-\alpha} .
$$

Using (3.13) for $c_{\alpha}(t)$, we have

$$
\sum_{\alpha} c_{\alpha}(t) v_{\alpha}(t)=\sum_{\alpha} \sum_{n} c_{\alpha, n} v_{\alpha}(t) \exp (2 \pi i n \ln t / \ln \Lambda)
$$

and $\ln \Lambda=\varepsilon g^{\prime}(0) / q$ in lowest order. We next assert that the combination $\sum_{\alpha} c_{\alpha}(t) v_{\alpha}(t)$ which appears in $s(r(t))-u(t)$ (see (3.12)) must be at largest of order $\xi^{0}$ near $t_{s}$. The argument is that $u(t)$ and $s(r(t))$ only get algebraically large in $\varepsilon$ in that region (see discussion below), so the difference cannot include, say, a $\xi^{-1}$ term which is exponentially large in $\varepsilon$. Here, we must also ensure that the quadratic terms of (3.12) remain smaller than $u(t)$ in the matching region. We present arguments for their smallness in Appendix $D$.

As long as $c_{\alpha}(t)$ is analytic, the $\alpha, n$ term in (4.6) falls off exponentially fast in $n$; hence no cancellation is possible, and we can bound (4.6) term by term. In Appendix $\mathrm{D}$, we conjecture that $c_{\alpha}(t)$ is analytic up to the matching region. Then, to leading 
order, the $\alpha, n$ term in (4.6) is of order 1 near the singularities $t_{s}$ and $t_{s}^{*}$, and we must have, respectively,

$$
\begin{aligned}
& 1 \gtrsim\left|c_{\alpha, n}\right| \xi^{q n} \xi^{\alpha}, \\
& 1 \gtrsim\left|c_{\alpha, n}\right| \xi^{-q n} \xi^{-\alpha},
\end{aligned}
$$

since $|\alpha| \leq q / 2$ we find that

$$
\begin{aligned}
& \left|c_{\alpha, n}\right| \lesssim \xi^{q|n|+\alpha \operatorname{sign}(n)}, \quad \text { for } n \neq 0 \\
& \left|c_{\alpha, 0}\right| \lesssim \xi^{|\alpha|} .
\end{aligned}
$$

This analysis permits only $\left|c_{0,0}\right|$ to be of order unity ${ }^{24}$. However, we have chosen (see $(3.14 b)$ ) the parameterization $r(t)$ to force this term to be zero. The next biggest terms are

$$
\begin{aligned}
\left|c_{ \pm 1,0}\right| \lesssim \xi, & \text { for } q>2, \\
\left|c_{1,0}\right|,\left|c_{1,-1}\right| \lesssim \xi, & \text { for } q=2 .
\end{aligned}
$$

All other terms are at most of order $\xi^{2}$ and are negligible in comparison to these.

It follows from (4.7) that if $\alpha \neq q / 2$ then $c_{\alpha} \sim c_{\alpha, 0}$; hence, $c_{\alpha}$ is essentially independent of $t$ (this observation is supported by the numerical calculations as shown in Figure 8). Moreover,

$$
\left|c_{\alpha}\right| \sim\left|c_{ \pm 1}\right| \alpha \mid
$$

When $\alpha=q / 2,(3.14)$ and (4.7) imply that

$$
\begin{aligned}
c_{q / 2}(t) v_{q / 2}(t) & \sim 2\left|c_{q / 2,0}\right| \cos \left(\pi \frac{\ln t}{\ln \Lambda}+\theta\right) \tilde{v}_{\alpha}(t)(1+O(\varepsilon)) \\
& \sim 2\left|c_{1,0}\right|^{q / 2} \cos \left(\pi \frac{\ln t}{\ln \Lambda}+\theta\right) \tilde{v}_{\alpha}(t)(1+O(\varepsilon))
\end{aligned}
$$

where $\theta$ is an unknown phase.

Now we are in a position to estimate to exponential order, the distance between the two curves. For $q>2$ we have

$$
\delta(t)=\|S(r(t))-U(t)\|=\left\|c_{1,0} V_{1}(t)+c_{1,0}^{*} V_{1}^{*}(t)\right\| .
$$

Substituting (3.8) and (3.16b) for $V_{1}(t)$, and $t=1+O(\varepsilon)$ in (4.11a), we find

$$
\delta(t) \sim \sqrt{2 q}\left|c_{1}\right|\left\{\frac{g\left(u_{0}(1)\right)}{g^{\prime}(0)}\right\}^{\cos (2 \pi p / q)}(1+O(\varepsilon)) .
$$

If $q=2$ and $t=1+O(\varepsilon)$,

$$
\delta(t)=\left\|V_{1}(1)\right\|\left|t^{x_{1}}\left(c_{1,0}+c_{1,-1} \exp \left[-2 \pi i \frac{\ln t}{\ln \Lambda}\right]\right)\right| ;
$$

hence, 


$$
\delta(t) \sim 2\left|c_{1,0}\right| \frac{g^{\prime}(0)}{g\left(u_{0}(1)\right)}\left|\cos \left(\pi \frac{\ln t}{\ln \Lambda}+\theta\right)\right|(1+O(\varepsilon))
$$

where $\theta$ is an unknown phase angle.

To complete this exponential order analysis, all that remains is to compute the phases, $\phi_{s}\left(-\phi_{s}\right)$, at which the singularities appear. To do this we have to solve

$$
\begin{aligned}
t \frac{d u}{d t} & =\frac{g(u(t))}{g^{\prime}(0)} \\
\frac{u(t)}{t} & \rightarrow 1 \quad \text { as } t \rightarrow 0
\end{aligned}
$$

Consider

$$
g(x)=x\left(1-x^{m}\right)
$$

where $m$ is a positive integer. The solution to (4.13) is

$$
u_{0}(t)=\frac{t}{\left(1+t^{m}\right)^{1 / m}}
$$

and $u$ approaches infinity as $t^{m}$ approaches -1 ; hence,

$$
\phi_{s}=\pi / m \text {. }
$$

Next take

$$
g(x)=\frac{\sin \pi x}{\pi}
$$

for which

$$
u_{0}(t)=\frac{2}{\pi} \arctan \left(\frac{\pi}{2} t\right)
$$

Here the singularities with smallest phase lie at $t= \pm(2 / \pi) i$, so

$$
\phi_{s}=\pi / 2 \text {. }
$$

To get a nontrivial case take

$$
g(x)=x(1-x)(1+a x), \quad-1<a<0, \quad 0<a<\infty,
$$

to find the implicit equation for $u$

$$
t^{1+a}=\frac{u_{0}^{1+a}}{\left(1-u_{0}\right)\left(1+a u_{0}\right)^{a}}
$$

The $u_{0}(t)$ is singular at $t=a^{(-a / 1+a)}(-1)^{(-1 / 1+a)}$; hence, the singularity with the smallest phase gives 


$$
\phi_{s}=\left\{\begin{array}{lc}
\frac{\pi}{1+a}, & 0<a<\infty \\
\pi, & -1<a<0 .
\end{array}\right.
$$

Notice that for positive rational $a, u_{0}(t)$ has a finite number of singularities, whereas for positive irrational $a$, the union of all singular points of $u_{0}(t)$ on all its branches forms a circle. Nevertheless, the value of $\phi_{s}$ is insensitive to this structure and only depends upon singularities on the principal branch.

We summarize the properties of the singular solutions in Table 3.

\section{B. Algebraic Corrections for $\delta(t)$}

We estimate the magnitude of $u(t), s(r)$, and $\tilde{v}(t)$ near the singularity in the matching region. These estimates suggest that $u, s$, and $\tilde{v}_{\alpha}$ are enhanced at most algebraically in this region. In addition, they supply an algebraic correction to the split distance $\delta(t)$. Theoretically, this part is not easy to justify. Our basic claim is that for regions bounded away from the singularities, the regular expansion in $\varepsilon$ is a valid asymptotic expansion; hence on the borderline of the validity region, the leading-order term represents correctly the order of magnitude of the diverging series. This claim is supported by our numerical calculations, but is yet to be proven.

Recall that our matching region is the region in which the continuum limit fails, namely, where

$$
\varepsilon g\left(x_{m}\right) \sim x_{m}, \quad\left|x_{m}\right| \gg 1,
$$

where $\left|x_{m}\right|$ denotes the order of magnitude of $\left|x_{j}\right|$ in the matching region. Solving (4.17) (to leading order in $\varepsilon$ ) for our three examples gives

$$
\begin{aligned}
& x_{m} \sim \varepsilon^{-1 / m}, \\
& x_{m} \sim \frac{i}{\pi} \ln \varepsilon^{-1}, \\
& x_{m} \sim|a \varepsilon|^{-1 / 2} .
\end{aligned}
$$

Indeed, it is easy to verify that these values of $x_{m}$ are realized by $u_{0}\left(t_{m}\right)$ where $t_{m}=t_{s} e^{-i \varepsilon \phi_{1}}$ and $\phi_{1}$ is an order-1 positive number.

By (4.18), both $u\left(t_{m}\right)$ and $s\left(t_{m}\right)$ exhibit at most algebraic dependence on $\varepsilon$. Similarly, we use the lowest-order solution of $\tilde{v}_{\alpha}(t)$ to estimate its magnitude in the matching region. Substituting (4.17), (4.18), and the value of $t_{m}$ into (3.16b), we obtain, to leading order in $\varepsilon$

$$
\begin{aligned}
& \tilde{v}_{\alpha}\left(t_{m}\right) \sim e^{-i \pi \Omega^{\alpha p} / m} \varepsilon^{-(1+1 / m) \Omega^{\alpha p},} \\
& \tilde{v}_{\alpha}\left(t_{m}\right) \sim(1 / 2)^{\Omega^{\alpha p}} \varepsilon^{-\Omega^{\alpha p}}\left(\ln \varepsilon^{-1}\right)^{\Omega^{\alpha p}}, \\
& \tilde{v}_{\alpha}\left(t_{m}\right) \sim a^{\Omega^{\alpha p}(a-1) / 2(a+1)} \varepsilon^{-3 / 2 \Omega^{\alpha p}} .
\end{aligned}
$$

Now we estimate the algebraic corrections to $\delta(t)$ by estimating $c_{\alpha}\left(t_{m}\right)$. By definition,

$$
\sum_{\alpha} c_{\alpha}\left(t_{m}\right) v_{\alpha}\left(t_{m}\right)=u\left(t_{m}\right)-s\left(r\left(t_{m}\right)\right) \leqq x_{m},
$$


and similarly,

$$
\sum_{\alpha} c_{\alpha}\left(t_{m}^{*}\right) v_{\alpha}\left(t_{m}^{*}\right) \lesssim x_{m}
$$

Using the same arguments as in the previous subsection, we obtain

$$
\begin{aligned}
\left|c_{\alpha, n}\right| & \lesssim \frac{\left|x_{m}\right| \xi^{q|n|+\alpha \operatorname{sign}(n)}}{\left|\tilde{v}_{\alpha}\left(t_{m}\right)\right|}, \quad n \neq 0, \\
\left|c_{\alpha, 0}\right| & \lesssim \frac{\left|x_{m}\right| \xi^{|\alpha|}}{\left|\tilde{v}_{\alpha}\left(t_{m}\right)\right|}
\end{aligned}
$$

Specifically, for the three examples we consider, the dominant term, $\left|c_{1,0}\right|$ is given by

$$
\begin{aligned}
\left|c_{1,0}\right| & \sim B \xi \varepsilon^{-1 / m+(1+1 / m) \cos (2 \pi p / q)} e^{O(\varepsilon)}, \\
\left|c_{1,0}\right| & \sim B \xi \varepsilon^{\cos (2 \pi p / q)} \exp \{O[\ln (\ln \varepsilon)]\}, \\
\left|c_{1,0}\right| & \sim B \xi \varepsilon^{-1 / 2+3 / 2 \cos (2 \pi p / q)} e^{O(\varepsilon)},
\end{aligned}
$$

where $B$ is an unknown constant (numerically $B$ is found to be a rather large constant, typically $B \sim 10^{3}$ ). It follows from (4.11) and (4.12) that the algebraic corrections to $\delta(t)$ for $t \sim 1$ are identical to the algebraic corrections to $\left|c_{1,0}\right|$ and they are given by (4.21). Hence, the coefficient $\gamma$ in (1.9) is given by the power of the $\varepsilon$ term in (4.21). We summarize the results for the coefficients in the asymptotic expansion of $\delta(t)$ in Table 1.

The occurrence of large constants such as $B$ is not surprising. We have neglected the order-1 terms in the exponents, which may contribute large numbers. For example, we know that $\left|c_{1,0}\right|$ is proportional to $t_{s}^{-\chi^{1}}$, which contains the term $-2 \pi\left|\phi_{s}\right|\left(\frac{1}{2}-p / q\right)$ (see (3.15a)) in its exponent. This term by itself can explain a $10^{3}$ factor in (4.21) for $p / q<\frac{1}{2}$. Numerically, we do not find a significant difference between the constants corresponding to $p / q<\frac{1}{2}$ and $p / q>\frac{1}{2}$. This suggests that there are other constants like the one mentioned here which were not accounted for. Also, we note that Lazutkin et al. ${ }^{21}$ find a similar behavior for the standard mapping.

\section{Estimates of $\delta_{0}$ and $\delta_{1}$}

We use the estimates of $c_{\alpha}$ 's to find $\delta_{0}$ and $\delta_{1}$. Equation (3.12) enables us to estimate the behavior of $S(r(t))$ as $t \rightarrow 0$. In this limit, $u(t) \rightarrow t$ and $v_{\alpha}(t) \rightarrow t^{\chi_{\alpha}}$, so that (3.17) implies

$$
S(r(t)) \approx t V_{0}+\sum_{\alpha \neq 0} c_{\alpha}(t) t^{\chi_{\alpha}} V_{\alpha}
$$

with $V_{\alpha}$ given in (2.6). In (4.22) we neglect the quadratic terms, which are of order $\left|c_{1,0}\right|^{2}$. It follows that ${ }^{24}$ the estimates for $\delta_{0}$ and $\delta_{1}$ are consistent for $q \leq 5$. Note that in the limit $\varepsilon \rightarrow 0$, the $\Lambda_{\alpha}$ reduce to $\Omega^{\alpha}$ so $\operatorname{Re}\left\{V_{\alpha}\right\}$ form an orthogonal set

$$
V_{\alpha} \cdot V_{\beta}^{T}=\left\{\begin{array}{ll}
q+O(\varepsilon), & \alpha=-\beta, \\
O(\varepsilon), & \text { otherwise. }
\end{array} \quad \alpha=\beta=q / 2,\right.
$$


There are two cases to be considered.

1. Orientation-preserving maps ( $q$ is odd): Then, as we argued in Section IV.A, the $c_{\alpha}(t)$ 's are essentially independent of $t$. Equation (4.22) implies that to leading order in $\varepsilon$

$$
\frac{\|S(r(t))\|^{2}}{q}=t^{2}+2 \sum_{\alpha=1}^{[q / 2]}\left|c_{\alpha}\right|^{2} t^{2 \operatorname{Re}\left\{\chi_{\alpha}\right\}}
$$

Using (3.11), (4.24a), and $b_{0}(t) \sim b_{0}$ for real $t$, we find

$$
\frac{\|S(r)\|^{2}}{q}=\left(b_{0} / r\right)^{2 \ln \Lambda / \ln \Gamma}+2 \sum_{\alpha=1}^{[q / 2]}\left|c_{\alpha}\right|^{2}\left(b_{0} / r\right)^{2 \operatorname{Re}\left\{\ln \Lambda_{\alpha} / \ln \Gamma\right\}}
$$

Equation (4.24) attains an extremum if

$$
t \sim-2 \sum_{\alpha=1}^{[q / 2]} \operatorname{Re}\left\{\chi_{\alpha}\right\}\left|c_{\alpha}\right|^{2} t^{2 \operatorname{Re}\left\{\chi_{\alpha}\right\}-1}
$$

We assume that the right-hand side of $(4.25)$ has a dominant term, so that

$$
\begin{aligned}
t & \sim\left[-2 \operatorname{Re}\left\{\chi_{\tilde{\alpha}}\right\}\left|c_{\tilde{\alpha}}\right|^{2}\right]^{1 /\left[2\left(1-\operatorname{Re}\left\{\chi_{\tilde{\alpha}}\right\}\right)\right]} \\
& \sim K_{\tilde{\alpha}}\left|c_{1}\right|^{\tilde{\alpha} /\left(1-\operatorname{Re}\left\{\chi_{\tilde{\alpha}}\right\}\right)}
\end{aligned}
$$

for some $\tilde{\alpha}, 0<\tilde{\alpha}<q / 2$. It is easy to show that the above result is consistent if $\tilde{\alpha}$ minimizes the expression $\alpha /\left[1-\operatorname{Re}\left\{\chi_{\alpha}\right\}\right]$. Since $t$ is positive, we also require $\operatorname{Re}\left\{\chi_{\tilde{\alpha}}\right\}<0$. Substituting (4.26) in (4.24), and using (4.9), we obtain

$$
\delta_{0} \sim A_{0}\left|c_{1}\right|^{\eta_{0}}, \quad \eta_{0}=\min _{\operatorname{Re} \alpha \alpha\}<0} \frac{\alpha}{1-\operatorname{Re}\left\{\chi_{\alpha}\right\}},
$$

where $A_{0}$ is a constant to leading order in $\varepsilon$.

In Figure $7 a$ we plot the distance $\|S(r)\|$ for $r$ values which are close to $r_{\min }$, where $r_{\min }=r(t)$ and $t$ is defined by (4.26). For $r<r_{\min },\|S(r)\|$ is dominated by the first term in (4.24b); hence, it is monotonically decreasing with $r$. For $r \gtrsim r_{\min }$, the dominant term is, to leading order in $\varepsilon$, monotonically increasing with $r$. The oscillations seen in the graph for $r \geq r_{\min }$ correspond to the first-order corrections to this dominant term. In Figure $7 \mathrm{~b}$ we present the analogous picture for $U(t)$.

Similar analysis gives

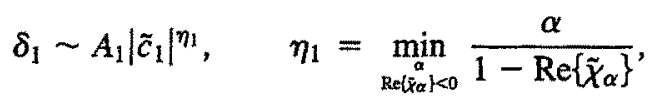

where

$$
\tilde{\chi}_{\alpha}=\frac{\ln \Gamma_{\alpha}}{\ln \Gamma}
$$


and $A_{1}$ is a constant (different from $A_{0}$ ) to leading order in $\varepsilon$. The value $\left|\tilde{c}_{1}\right|$ is found from the relation

$$
\delta(t) \sim\left|\tilde{c}_{1} W_{1}(r(t))\right| \sim\left|c_{1} V_{1}(t)\right|
$$

which at $t=1$ gives, to leading order in $\varepsilon$,

$$
\left|\tilde{c}_{1}\right|=\left|c_{1}\right| \text {. }
$$

Here, $\tilde{c}_{1} W_{\alpha}(r)$ is the analogue of $V_{\alpha}(t)$, namely, it is the nonlinear extension of $W_{\alpha}$ in (2.14). In Table 2 we show the numerical and theoretical values we get for $\eta_{0}$ and $\eta_{1}$ for various $p$ 's and $q$ 's.

2. Orientation-reversing maps ( $q$ is even): Then, for $\alpha \neq q / 2, c_{\alpha}(t)$ 's are essentially independent of $t$, but $c_{q / 2}(t) t^{x_{q / 2}}$ oscillates. Equations (4.9), (4.10), and (4.22) imply that

$$
\frac{\|S(r(t))\|^{2}}{q}=t^{2}+2 \sum_{\alpha=1}^{[q / 2-1]}\left|c_{\alpha}\right|^{2} t^{2 \operatorname{Re} \chi_{\alpha}}+\left|c_{1}\right|^{q} t^{-2} \cos ^{2}\left(\pi \frac{\ln t}{\ln \Lambda}+\theta\right)
$$

where $\theta$ is a constant. Hence, $\|S(r(t))\|$ oscillates in $t$ even in the leading-order terms. As $t \rightarrow 0$, the last term in (4.29) dominates and grows without bound for almost all $t$. However, there exists a subsequence $t_{n} \rightarrow 0$ for which this term vanishes. If Re $\chi_{\alpha}>0$ for all $0<\alpha<q / 2$, this implies that, to linear order, $\left|S\left(r\left(t_{n}\right)\right)\right| \rightarrow 0$ as $t_{n} \rightarrow 0$, namely, that heteroclinic orbits possibly exist. These conditions are satisfied for $q=2$ and for $q=4$ with $p=1$. The possible existence of heteroclinic orbits in fourdimension, created via intersections of one-dimensional curves, is quite surprising. Numerically we have found similarities between the $q=4, p=1$, and the $q=2$ cases, but we cannot conclude whether we have found heteroclinic orbits. With these observations in mind, the calculation of the minimal distance of approach, when there are no heteroclinic orbits, is straightforward: one considers (formally) the sequence $t_{n}$ for which the last term vanishes, and therefore disregards this term, obtaining similar results to the orientation-preserving case. When there exists heteroclinic orbit, one has to redefine the meanings of $\delta_{0}$ and $\delta_{1}$. For example, for $q=2(4.29)$ becomes

$$
\begin{aligned}
\frac{\|S(r(t))\|^{2}}{2} & =t^{2}+\left|c_{1}\right|^{2} t^{-2} \cos ^{2}\left(\pi \frac{\ln t}{\ln \Lambda}+\theta\right) \\
& =\left(b_{0} / r\right)^{2 \ln \Lambda / \ln \Gamma}+\left|c_{1}\right|^{2}\left(r / b_{0}\right)^{2 \ln \Lambda / \ln \Gamma} \cos ^{2}\left(\pi \frac{\ln r}{\ln \Gamma}+\theta^{\prime}\right) .
\end{aligned}
$$

As $r \rightarrow \infty$, the second term in (4.30) dominates, and $\|S(r)\|$ oscillates wildly; there is one subsequence of $r$ values for which $\|S(r)\| \rightarrow 0$ (corresponding to the heteroclinic orbits) and for almost all other points $\|S(r)\| \rightarrow \infty$. Therefore, we define $\delta_{0}$ to be the minimal ${ }^{7}$ distance of the envelope of $\|S(r(t))\|$ from the origin, namely,

$$
\delta_{0}=\min _{j} \max _{t_{0}<t \leq \Lambda t_{0}}\left\|S\left(r\left(\Lambda^{-j} t\right)\right)\right\|
$$


where $t_{0}$ is some fixed $O\left(10^{-1}\right)$ quantity (clearly $\delta_{0}$ is independent of $\left.t_{0}\right)$. Using (4.29) and (4.31), we obtain exactly the same result as in (4.27), although the interpretation of $\delta_{0}$ and $\delta_{1}$ is different. In Figure 9 we plot $\|S(r)\|$ for $q=2, g(x)=x(1-x)$, and $\varepsilon=0.75$. The oscillation in $\|S(r)\|$ is clearly seen. The envelope of $\|S(r)\|$ and $\delta_{0}$ are also shown in the figure.

\section{Numerical Results}

We test our estimates of $\delta\left(t_{1}\right), \delta_{0}, \delta_{1}$, and $c_{1}$ by computing these quantities numerically for a range of $\varepsilon$ values and comparing the exponents and power law dependencies on $\varepsilon$ with the predictions. The results of these computations are summarized in Tables 2 and 4. In Table 4 we present the theoretical and numerical values of $\beta$ and $\gamma$ for several forms of $g(x)$, where

$$
\left|c_{1,0}\right| \sim B \varepsilon^{\gamma} e^{-\beta / \varepsilon}
$$

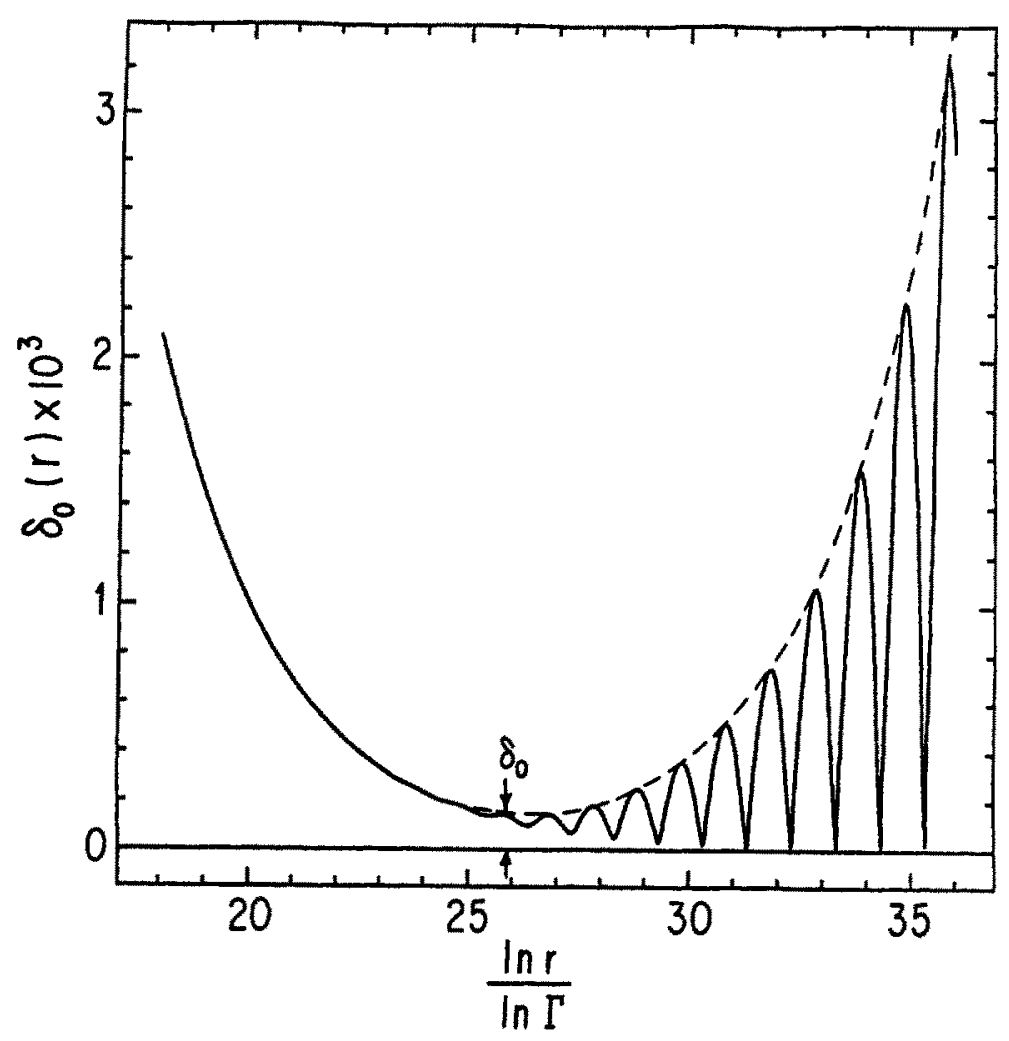

Fig. 9. The definition of the miss distance from the fixed points in the twodimensional case $(q=2)$. We plot $\delta_{0}(r)$ as a function of $\ln r / \ln \Gamma$ for $g(x)=x(1-x), q=2$, and $\varepsilon=0.75$. The miss distance $\delta_{0}$ is defined as the minimum of the envelope of $\delta_{0}(r)$ which is drawn schematically in the figure. Note that the minima of $\delta_{0}(r)$ correspond to heteroclinic orbits and asymptotically approach 0 as $r \rightarrow \infty$. 
Beyond All Orders: Singular Perturbations in a Mapping

Table 4 Theoretical and numerical values of $\beta$ and $\gamma$.

\begin{tabular}{|c|c|c|c|c|c|c|c|}
\hline Function, $g(x)$ & $\mathrm{m}$ or $\mathrm{a}$ & $q$ & $p$ & $\beta_{\text {the }}$ & $\beta_{\text {num }}$ & $\gamma_{\text {the }}$ & $\gamma_{\text {num }}$ \\
\hline \multirow{6}{*}{$x\left(1-x^{m}\right)$} & \multirow{2}{*}{1} & 3 & 2 & 19.739 & 19.718 & -2.000 & -2.001 \\
\hline & & 5 & 3 & 19.739 & 19.710 & -2.618 & -2.587 \\
\hline & \multirow{2}{*}{2} & 3 & 2 & 9.870 & 9.860 & -1.250 & -1.259 \\
\hline & & 5 & 3 & 9.870 & 9.863 & -1.714 & -1.703 \\
\hline & \multirow{2}{*}{3} & 3 & 2 & 6.580 & 6.574 & -1.000 & -1.008 \\
\hline & & 5 & 3 & 6.580 & 6.572 & -1.412 & -1.409 \\
\hline \multirow{16}{*}{$x(1-x)(1+a x)$} & \multirow{2}{*}{$-\frac{\pi}{5}$} & 3 & 2 & 19.739 & 19.732 & -1.250 & -1.250 \\
\hline & & 5 & 3 & 19.739 & 19.732 & -1.714 & -1.710 \\
\hline & \multirow{2}{*}{$-\frac{1}{2}$} & 3 & 2 & 19.739 & 19.732 & -1.250 & -1.250 \\
\hline & & 5 & 3 & 19.739 & 19.730 & -1.714 & -1.710 \\
\hline & \multirow{2}{*}{$\frac{1}{2}$} & 3 & 2 & 13.160 & 13.153 & -1.250 & -1.224 \\
\hline & & 5 & 3 & 13.160 & 13.150 & -1.714 & -1.683 \\
\hline & \multirow{2}{*}{$\frac{2}{3}$} & 3 & 2 & 11.844 & 11.837 & -1.250 & -1.238 \\
\hline & & 5 & 3 & 11.844 & 11.834 & -1.714 & -1.694 \\
\hline & \multirow{2}{*}{$\frac{1+\sqrt{5}}{4}$} & 3 & 2 & 10.912 & 10.905 & -1.250 & -1.245 \\
\hline & & 5 & 3 & 10.912 & 10.902 & -1.714 & -1.701 \\
\hline & \multirow{2}{*}{$\frac{\pi}{4}$} & 3 & 2 & 11.056 & 11.049 & -1.250 & -1.243 \\
\hline & & 5 & 3 & 11.056 & 11.046 & -1.714 & -1.700 \\
\hline & \multirow{2}{*}{1.5} & 3 & 2 & 7.896 & 7.889 & -1.250 & -1.266 \\
\hline & & 5 & 3 & 7.896 & 7.886 & -1.714 & -1.725 \\
\hline & \multirow{2}{*}{$\pi$} & 3 & 2 & 4.766 & 4.763 & -1.250 & -1.269 \\
\hline & & 5 & 3 & 4.766 & 4.762 & -1.714 & -1.725 \\
\hline$\frac{\sin \pi x}{\pi}$ & & $\begin{array}{l}3 \\
5\end{array}$ & $\begin{array}{l}2 \\
3\end{array}$ & $\begin{array}{l}9.870 \\
9.870\end{array}$ & $\begin{array}{l}9.867 \\
9.865\end{array}$ & $\begin{array}{l}-0.500 \\
-0.809\end{array}$ & $\begin{array}{l}-0.506 \\
-0.800\end{array}$ \\
\hline
\end{tabular}


and $B$ is a constant. Notice the agreement (typically three significant digits) between the numerical values and the theoretical predictions for all dimensions (different $q$ 's and $p$ 's) and for all the different functions we considered. For the function $g(x)=$ $x(1-x)(1+a x)$ the structure of the singularities of $u_{0}(t)$ depends sensitively on the value of $a$; see (4.16). We demonstrate that the theoretical prediction appears to work equally well for values of $a$ which are negative, positive, rational, and irrational (using the computer, double-precision approximation of irrationals).

The numerical computations for Table 4 are performed in the complex plane. There, we can compute for $\varepsilon$ values as small as $O\left(10^{-3}\right)$. We think that the exponents we see reflect the $\varepsilon \rightarrow 0^{+}$limit. In addition, the computation in the complex plane enables us to check many of the assertions in this paper. For example, we perform the numerical computation in the matching region where $\phi=\phi_{s}-\varepsilon \phi_{1}$ and find that for $\phi_{1}$ sufficiently large (typically $\phi_{1} \approx O\left(\phi_{s}\right)$ ), the coefficient $c_{1}(t)$ is essentially independent of $t$ to order $c_{1}^{2} v_{1}^{2}$; hence the quadratic terms in (3.12) are indeed small, and the sum over $\alpha, n$ in (4.6) is dominated by the lowest-order terms. In Appendix $\mathrm{C}$, we present detailed analysis of these effects. In addition, we find that $u, s$, and $\tilde{v}_{\alpha}$ are of the predicted order of magnitude in the matching region.

In Table 2 we present the theoretical and numerical values of $\eta_{0}$ and $\eta_{1}$ for the maps $g(x)=x\left(1-x^{m}\right), m=1,2$, where

$$
\begin{aligned}
& \delta_{0} \sim A_{0}\left|c_{1}\right|^{\eta_{0}}, \\
& \delta_{1} \sim A_{1}\left|c_{1}\right|^{\eta_{1}},
\end{aligned}
$$

and $A_{0}$ and $A_{1}$ are constants to order $e^{O(\varepsilon)}$. The numerical results are in satisfactory agreement with the theoretical predictions (typically the results agree to two significant digits). The results are not as sharp as for the asymptotic coefficients of $\left|c_{1}\right|$; the computations of $\delta_{0}$ and $\delta_{1}$ are limited to quite large $\varepsilon$ values, and at these values of $\varepsilon$ higher-order terms may be significant (see Table A.2).

Finally, in Figure 10 we display a test of the relation between $\delta(t)$ and $\left|c_{1}\right|$ (see $(4.11 b))$ for $t=t_{1}$ for the case $g(x)=x(1-x), q=3$, and $p=2$. Equation $(4.11 b)$ does seem to work.

\section{E. Numerical Method}

The quantities $\delta(t), \delta_{0}, \delta_{1}$, and $c_{1,0}$ are extremely small for small values of $\varepsilon$. Hence, we developed a careful numerical scheme for computing these quantities. Here we describe the numerical scheme, and in Appendix $C$ we derive estimates for the numerical errors.

To compute $\delta\left(t_{1}\right)$ and the $c_{\alpha}$ 's we find, using a Newton method, the value of $t_{0}$ such that $\Lambda^{j} t_{0}=t_{1}$ and $t_{0}$ is about 0.2 (recall that $u\left(t_{1}\right)=u_{0}(1)$ ). Then, we initialize $u\left(t_{0}\right)$ and $\tilde{v}\left(t_{0}\right)$. To obtain accurate initial conditions, we always use the Taylor expansion (of $u, \tilde{v}$, and $s$, as described in Appendix B), taking enough terms so that the corrections are zero to the machine accuracy $\left(10^{-15}\right)$. Then, we advance $u$ and $\tilde{v}, j$ times according to (2.9) and (3.9), respectively. Using a Newton method once more, we determine the value of $r_{0}$ for which the vector $\left(U\left(t_{1}\right)-S\left(r_{1}\right)\right)$ is perpendicular to $V_{0}\left(t_{1}\right)$, where $r_{1}=r_{0} / \Gamma^{k}, k$ is $O(1)$, and $r_{0}$ is about 0.2 . Then, 


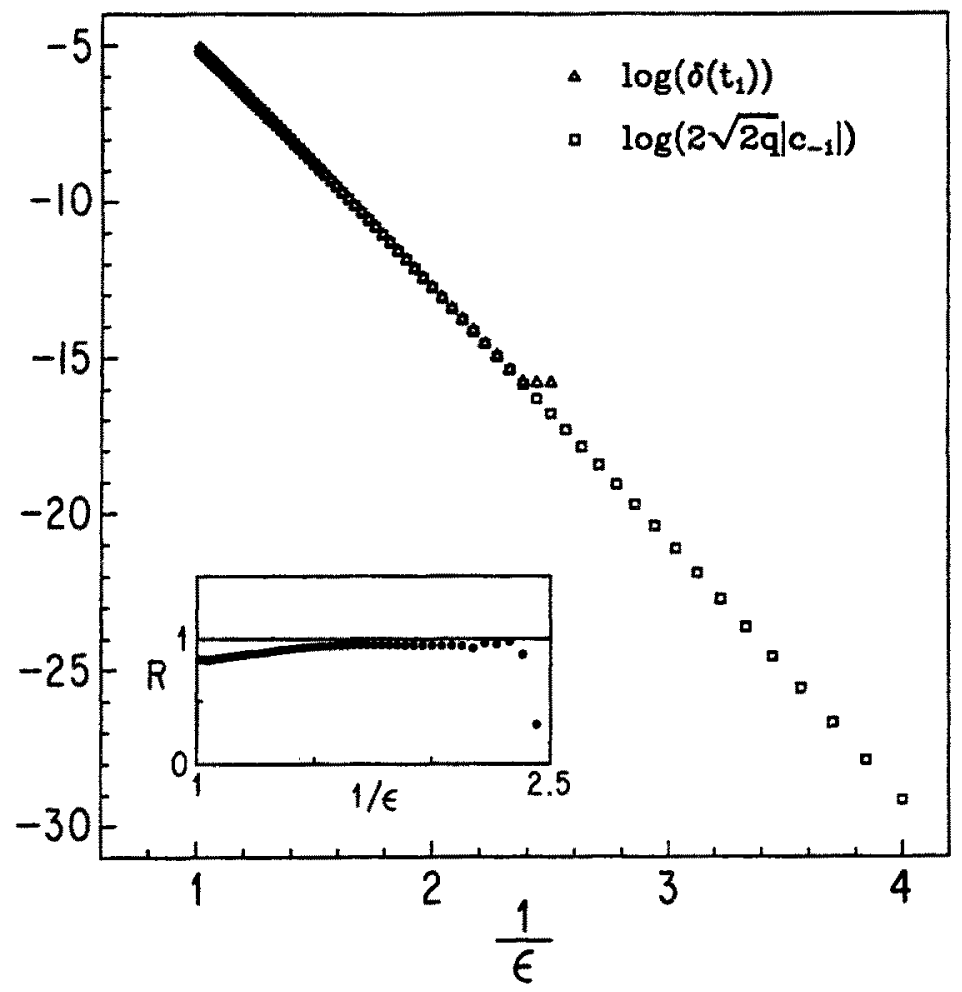

Fig. 10. Comparison of the miss distance $\delta\left(t_{1}\right)$ and $2 \sqrt{2 q}\left|c_{-1}\right|,((4.11 b))$ for the case $g(x)=x(1-x), q=3$, and $p=2$. The agreement between the two quantities is clearly seen for sufficiently large $\varepsilon$ values. The advantage of working with the coefficient $\left|c_{-1}\right|$ rather than the miss distance is also demonstrated in the figure: we can compute $\left|c_{-1}\right|$ for very small $\varepsilon$ values, whereas the computation of $\delta\left(t_{1}\right)$ fails for $\varepsilon \leq 0.4$. In the inset we plot the ratio of the two quantities, $R=2 \sqrt{2 q}\left|c_{-1}\right| / \delta\left(t_{1}\right)$, versus $1 / \varepsilon$ for $0.4<\varepsilon<1$. Equation $(4.11 b)$ implies that $R=1+O(\varepsilon)$. Numerically, $R \sim 0.9 \pm 0.1$ for $\varepsilon>0.45$ whereas for smaller $\varepsilon$ values the computation of $\delta\left(t_{1}\right)$ becomes less accurate and $R(\varepsilon)$ oscillates.

$\delta\left(t_{1}\right)$ is given by the magnitude of $U\left(t_{1}\right)-S\left(r_{1}\right)$, and the values of the $c_{\alpha}$ 's are found by inverting the matrix spanned column-wise by the $V_{\alpha}$ 's. The only $c_{\alpha}$ 's for which our numerical results are significant are $c_{ \pm 1}$ since all the other $c_{\alpha}$ 's are contaminated by the quadratic powers of $c_{ \pm 1} V_{ \pm 1}$. In Appendix $C$ we show that the computation of $\delta\left(t_{1}\right)$ is accurate to machine accuracy, and that the computation of $\left|c_{1}\right|$ is accurate to the machine accuracy or up to the magnitude of the quadratic terms, whichever is larger.

The above procedure works well for $\varepsilon \gtrsim 0.45$ (for the quadratic case with $q=3, p=2$ ). For smaller values of $\varepsilon$ the magnitude of $\delta\left(t_{1}\right)$ vanishes to machine accuracy. However, to get sharp values for $\beta$ and $\gamma$ we must obtain results for a larger range of $\varepsilon$ 's. Therefore, we perform a similar procedure in the matching region 
of the complex $t$-plane, where $\delta(t)$ is increased algebraically as $\varepsilon$ decreases. First, we fix the phase of $t_{c}$ (the point at which the stable and unstable manifolds are matched) to be $\phi_{s}-\varepsilon \phi_{1}$, where $\phi_{1}$ is an order-1, positive constant. The magnitude of $t_{c}$ is determined so that $\left|t_{c}-t_{s}\right|$ is minimized, giving

$$
\left|t_{c}\right|=\left|t_{s}\right| \cos \varepsilon \phi_{1} .
$$

We initialize $U$ and $\tilde{V}$ at $t_{0}=\left|t_{c}\right| \exp \left[i\left(\phi_{s}-\varepsilon \phi_{1}\right)\right] \Lambda^{-j}$, where $j$ is an order-1 integer for which $\left|t_{0}\right|$ is about 0.2 . Then we advance $u$ and $\tilde{v}$ and determine $r_{c}$ as in the scheme for real $t$ and $r$. We use (3.19) to obtain an initial guess to the phase of $r_{c}$. We note that in the complex plane it is important to iterate $\tilde{v}(t)$ rather than $v(t)$, since the term $t^{\chi_{\alpha}}$ is exponentially small (or large). Once $r_{c}$ is found, we compute $\delta\left(t_{c}\right)$. To obtain the $c_{\alpha}$ 's we need to invert the matrix of the $V_{\alpha}$ 's. To avoid their exponential smallness/largeness we normalize $t$ by $t_{\text {norm }}$, where $t_{\text {norm }}=\exp \left(i\left(\phi_{s}-\varepsilon_{s} \phi_{1}\right)\right)$, and $\varepsilon_{s}$ is the smallest $\varepsilon$ value we use in the computation. Once the normalized $c_{\alpha}$ 's are found, we multiply them by $\left(t_{s} / t_{\text {norm }}\right)^{X_{\alpha}}$. The result of the computation is $\left(t_{s}\right)^{X_{\alpha}} c_{\alpha}$, which enables us to check directly the correctness of $\gamma$ without computing exponentially small (or large) quantities. To measure $\beta$ we use the results of the small $\varepsilon$ range, together with an appropriate normalization. It follows from the numerical error analysis that there is a range of $\phi_{1}$ values for which $\|\Delta(t)\|$ is sufficiently large and the quadratic corrections are sufficiently small so that $c_{ \pm 1}$ can be determined up to seven significant digits.

The numerical computation of $\delta_{0}$ and $\delta_{1}$ is done for real $t$, in a similar fashion to the computation of $\delta\left(t_{1}\right)$. One merely replaces the Newton method by a minimization scheme on the interval $\left[t_{0}, \Lambda^{q} t_{0}\right)$, where $t_{0}=O\left(10^{-1}\right)$. However, in this case we are limited not only by the smallness of $\delta_{0}$ and $\delta_{1}$ but also by the accumulating numerical error, since, for small $\varepsilon$, we iterate (1.1) many times. In Appendix $C$ we estimate the minimal value of $\varepsilon$ beyond which our results are significant: the critical $\varepsilon$ at which the error term and $\delta_{0}$ (resp. $\delta_{1}$ ) are of the same order of magnitude. These lower bounds agree with the lowest value of $\varepsilon$ for which our numerical scheme converges as shown in Table A.2. As $\varepsilon$ is increased, the relative error decreases exponentially in $1 / \varepsilon$.

\section{Summary and Conclusions}

We have developed a method for estimating the distance between the stable and unstable curves for a family of $q$-dimensional maps. It uses the singularities in the complex plane of the solution to the limiting flow, $u(t)$, and the behavior of the linearized modes, $v_{\alpha}(t)$, near the singularities. To find the distance between the curves we first find the $\varepsilon$-dependence of $u(t)$ and $v_{\alpha}(t)$ in the vicinity of the "closest" singularity. This behavior determines the magnitude of the coefficients of the linearized modes, $\left|c_{\alpha}(t)\right|$. Using the theoretical expressions for $v_{\alpha}(t)$ and $\left|c_{\alpha}(t)\right|$, we compute the distance between the curves for any real $t$.

To test our predictions for the curve separation, we have developed numerical schemes which compute the curves, the $c_{\alpha}$ 's, the $v_{\alpha}$ 's, and the distance between the curves. The computations are done in the complex $t$-plane to achieve high accuracy for small values of $\varepsilon$ with a standard, double-precision workstation. We find excellent 
agreement between the numerical results and the theoretical predictions for all the examples we considered.

Generalizations and extensions of this work may include:

i) A rigorous proof that for entire $g(x)$ the stable and unstable curves cannot coincide or, on the contrary, a characterization of all the analytic functions for which such a phenomenon occurs.

ii) Characterization of the distance between invariant manifolds of higher dimensions (instead of one-dimensional curves).

iii) Investigation of the change in the distance between the curves as a result of small changes in $g$.

iv) A rigorous proof of the assertions in Appendix D.

\section{Acknowledgments}

We would like to thank P. Holmes, C. Simó, and S. Tanveer for their comments on an earlier version of the manuscript. We also thank S. Wiggins and J. Marsden for suggesting relevant references. The work of E. S. C. Ching, L. P. Kadanoff, and V. Rom-Kedar is supported by NSF DMR. The latter also acknowledges NSF Grant DMS8903244. C. Amick was supported by NSF Grant DMS8507784.

Note. This will be one of Charles Amick's last papers. He died on June 3, 1991. In our collaborations, Charles showed a rare mixture of good humor, rigorous dedication to truth, and tremendous enthusiasm for knowledge. We miss him.

\section{Appendix A: Existence and Uniqueness of $U(t)$ and $S(r)$}

Here we prove Proposition 1 of Section I.

Proposition 1. For every $\varepsilon>0$, there exist unique entire vector-valued functions $U(t)$ and $S(r(t)),\left(U: C \rightarrow C^{q}\right.$ and $\left.S: C \rightarrow C^{q}\right)$ which are solutions to the mapping problem in the sense that

$$
U(\Lambda t)=G(U(t))
$$

and

$$
S(r / \Gamma)=G(S(r))
$$

with the conditions that

$$
\begin{gathered}
U(0)=0, \quad S(0)=1, \\
\lim _{t \rightarrow 0} \frac{U(t)}{t}=\left(1, \Lambda, \ldots, \Lambda^{q-1}\right), \quad \lim _{r \rightarrow 0} \frac{S(r)-S(0)}{r}=-\left(1, \Gamma^{-1}, \ldots, \Gamma^{-q+1}\right) .
\end{gathered}
$$


Proof. We shall prove the part for $U(t)$; the proof for $S(r)$ is identical.

It follows from the definition of $G$ (see (1.7)) that Proposition 1 holds if and only if there exists a unique entire scalar function $u(t)$ defined by (2.9)

$$
\begin{aligned}
u\left(\Lambda^{q} t\right) & =u(t)+\varepsilon g\left(u\left(\Lambda^{p} t\right)\right), \\
u(0) & =0, \quad \lim _{t \rightarrow 0} \frac{u(t)}{t}=1 .
\end{aligned}
$$

Here $t$ is a complex variable, $\varepsilon>0$ is fixed, and $g$ is entire with $g(0)=0$ and $g^{\prime}(0)>0$. A similar argument holds for the function $S$. For ease of notation, we shall do the case of $g$ given by (4.14a) with $q=3, p=2$, and $m=1$. Analogous arguments hold for the general case. The idea of the proof is to invert the linear operator on the left-hand side of the equation

$$
u\left(\Lambda^{3} t\right)-u(t)-\varepsilon u\left(\Lambda^{2} t\right)=-\varepsilon u\left(\Lambda^{2} t\right)^{2}
$$

and solve the resulting equations by Banach's contraction mapping principle. For a suitably smooth function $h$ with $h(0)=h^{\prime}(0)=0$, the solution of the linear equation

$$
\begin{aligned}
u\left(\Lambda^{3} t\right)-u(t)-\varepsilon u\left(\Lambda^{2} t\right) & =h(t), \\
\lim _{t \rightarrow 0} \frac{u(t)}{t} & =1,
\end{aligned}
$$

is given by

$$
u(t)=t+\sum_{j=0}^{\infty} \Lambda^{j} \Lambda_{-1}^{m} \Lambda_{1}^{n} h\left(\Lambda^{-j-m-n-3} t\right)
$$

where $\Lambda>1, \Lambda_{1}, \Lambda_{-1}$ are the roots of (2.2), namely, the roots of $x^{3}-\varepsilon x^{2}-1=0$. Since $\Lambda\left|\Lambda_{1} \Lambda_{-1}\right|=\Lambda\left|\Lambda_{1}\right|^{2}=1$, it follows that $\left|\Lambda_{1}\right|=\left|\Lambda_{-1}\right|=1 / \sqrt{\Lambda}<1$. The formula giving $u$ in terms of $h$ is analogous to the standard variation of parameters formula for differential equations. For our problem, $h(t)=-\varepsilon u\left(\Lambda^{2} t\right)^{2}$, and setting $u(t)=t+R(t)$ yields

$$
R(t)=\eta(R)(t) \equiv-\varepsilon \sum_{0}^{\infty} \Lambda^{j} \Lambda_{-1}^{m} \Lambda_{1}^{n}\left[\Lambda^{-j-m-n-3} t+R\left(\Lambda^{-j-m-n-3} t\right)\right]^{2} .
$$

For each $\delta>0$, let $Z=Z(\delta)$ denote the set functions $R$ such that $R$ is analytic on the disc $\{t \in C ;|t|<\delta\}$, continuous on the closed disc $\{t \in C ;|t| \leq \delta\}$, and $R(0)=R^{\prime}(0)=0$. Denote the norm of $R$ by

$$
\|R\|=\max _{|t| \leq \delta}|R(t)|=\max _{|t|=\delta}|R(t)| .
$$

An elementary calculation shows that if $\delta$ is sufficiently small, then $\eta$ maps $Z(\delta)$ into itself and is a contraction there

$$
\|\eta(R)-\eta(W)\| \leq \frac{1}{2}\|R-W\|
$$

for all $R, W \in Z(\delta)$. Banach's contraction mapping theorem gives the existence of a 
unique $R \in Z(\delta)$ satisfying $R(t)=\eta(R)(t),|t|<\delta$. Since $R$ is analytic on this disc, the same is true of $u$. The form of the equation for $u$ ensures that it is entire.

Now we prove that for sufficiently large $\varepsilon$ the stable and unstable curves cannot coincide. Here we consider the case $g(x)=x(1-x), q=3$, and $p=2$. For any $\delta>0$, the maximum modulus principle gives

$$
\max _{|t| \leq \delta}|u(t)|=\max _{|t|=\delta}|u(t)|=-u(-\delta)
$$

the final equality follows from (B.2) since $a_{n}>0$ for all $n$. It follows from (B.2) that $-u(t), u^{\prime}(t), u^{\prime \prime}(t), \ldots$ are all positive on $(-\infty, 0)$ and tend to $\infty$ as $t \rightarrow-\infty$. One can show that the growth of $u$ is well approximated by the equation

$$
u\left(\Lambda^{q} t\right) \approx-\varepsilon u\left(\Lambda^{p} t\right)^{2}, \quad \text { as } t \rightarrow-\infty .
$$

This is analyzed easily, and one can find the order of $u$

$$
\limsup _{\delta \rightarrow \infty} \ln (\delta)^{-1} \ln \left\{\ln \left[\max _{|t| \leq \delta}|u(t)|\right]\right\}=\frac{\ln 2}{(q-p) \ln \Lambda} .
$$

A standard theorem in complex analysis ${ }^{25}$ states that an entire function of order $<\frac{1}{2}$ cannot be bounded on an arc connecting 0 to infinity. If we take $q=3, p=2$ for definiteness, then the condition $(\ln 2 / \ln \Lambda)<\frac{1}{2}$ is equivalent to $\varepsilon>\frac{63}{16}$. Hence, for this case, $u(t)$ does not remain bounded as $t \rightarrow \infty$. A little additional analysis shows that

$$
\liminf _{t \rightarrow \infty} u(t)=-\infty
$$

In particular, the stable and unstable curves cannot coincide for the quadratic case with $q=3, p=2$, and $\varepsilon>\frac{63}{16}$.

\section{Appendix B: Series Expansions}

In this section, we derive a series expansion for $u$ when $g$ is given by (4.14a) with $m=1$. We also give another proof that $u$ is entire.

Take $g$ to be of the quadratic form given by (4.14a) and $m=1$. Then (2.9a) reads

$$
u\left(\Lambda^{q} t\right)-u(t)-\varepsilon u\left(\Lambda^{p} t\right)=-\varepsilon\left[u\left(\Lambda^{p} t\right)\right]^{2} .
$$

Write $u(t)$ in a formal series expansion

$$
u(t)=\sum_{n=1}^{\infty} a_{n}(-1)^{n+1} t^{n}
$$

The coefficient of the first power of $t$ is

$$
a_{1}\left[\Lambda^{q}-1-\varepsilon \Lambda^{p}\right] .
$$

According to the definition of $\Lambda$, the factor in the square brackets vanishes. We now 
take $a_{1}=1$ and move on to consider higher-order terms which obey

$$
B_{k} a_{k}=\sum_{l=1}^{k-1} a_{l} a_{k-l}
$$

with

$$
B_{k}=\frac{\Lambda^{(q-p) k}-\Lambda^{-p k}-\varepsilon}{\varepsilon}
$$

for $k=1,2,3, \ldots$. Since $B_{k}$ is strictly an increasing function of $k$, and since $B_{1}=0, B_{k}$ is always positive for $k>1$. Then, (B.3) implies that all the $a_{k}$ 's are positive.

It follows from (B.1) that there are only two possible kinds of behavior for the formal series (B.2) (recall that $\Lambda>1$ ).

a. The series has a zero radius of convergence, whence $u(t)$ has a singularity at $t=0$, b. The series has an infinite radius of convergence.

We shall distinguish between the two possibilities by means of a ratio test in which the sequence of ratios

$$
r_{k}=\frac{a_{k+1}}{a_{k}}
$$

will be shown to have a finite upper bound. This finiteness eliminates possibility (a) and thus implies possibility (b).

The proof is constructed by taking the ratio of the terms in (B.3) for successive values of $k$. The ratio equation reads

$$
\frac{B_{k+1}}{B_{k}} r_{k}=\frac{\sum_{m=1}^{k} a_{m} a_{k-m+1}}{\sum_{n=1}^{k-1} a_{n} a_{k-n}}
$$

To use this result, define a coefficient ratio

$$
R_{k}=\frac{B_{k+1}}{B_{k}}
$$

and rewrite our result as

$$
R_{k} r_{k}=\frac{a_{k}+\sum_{m=1}^{k-1} a_{m} a_{k-m+1}}{\sum_{n=1}^{k-1} a_{n} a_{k-n}}
$$

and finally

$$
r_{k}=\frac{1}{B_{k+1}}+\frac{\sum_{m=1}^{k-1} a_{m} a_{k-m} r_{k-m}}{R_{k} \sum_{n=1}^{k-1} a_{n} a_{k-n}}
$$


Now we set some bounds. From its definition

$$
R_{k}>\Lambda^{q-p}>1
$$

for $k>1$, and consequently

$$
B_{k}>B_{2} \Lambda^{(q-p)(k-2)}
$$

for $k>2$. Furthermore, $B_{2}>\Lambda^{-p}$ so that

$$
B_{k}>\Lambda^{-p} \Lambda^{(q-p)(k-2)}
$$

for $k>2$. Now, let $M_{k}$ be the maximum value of $\left(r_{2}, r_{3}, r_{4}, \ldots, r_{k-1}\right)$. Since the $a$ 's are positive, (B.7) implies

$$
r_{k}<\Lambda^{p} \Lambda^{-(q-p)(k-1)}+M_{k} \Lambda^{-(q-p)} .
$$

Assume now that possibility (a) holds, i.e., $\lim \sup r_{k}$ is infinite. Then no matter how large $m$ might be there is a $k>m$ for which $r_{k}>M_{k}$. Then (B.10) implies that for these values of $k$

$$
M_{k}<\frac{\Lambda^{p} \Lambda^{-(q-p)(k-1)}}{1-\Lambda^{-(q-p)}}
$$

and thus there is a bound on $r_{k}$ which gets smaller and smaller as $k$ gets larger. Since we started by assuming that, for large $k, r_{k}$ was unbounded from above, we have reached a contradiction. We must conclude that $(b)$ is true.

A very similar approach will give a series for $v_{\alpha}(t)$, which obeys

$$
v_{\alpha}\left(\Lambda^{q} t\right)=v_{\alpha}(t)+\varepsilon g^{\prime}\left(u\left(\Lambda^{p} t\right)\right) v_{\alpha}\left(\Lambda^{p} t\right) .
$$

To state the boundary conditions we write, as in Section III,

$$
v_{\alpha}(t)=t^{\chi \alpha} \tilde{v}_{\alpha}(t)
$$

where $\chi_{\alpha}$ is given by (3.8) and $\tilde{v}_{\alpha}(0)=1$. Now we use an expansion like that in (B.2)

$$
\tilde{v}_{\alpha}(t)=\sum_{n=1}^{\infty} a_{n, \alpha}(-1)^{n-1} t^{n-1}
$$

As before, we take $a_{1, \alpha}=1$. Now (3.11) translates to the conditions on the expansion coefficients

$$
B_{k, \alpha} a_{k, \alpha}=\sum_{m=1}^{k-1} a_{m, \alpha} a_{k-m, \alpha}
$$

where 


$$
B_{k, \alpha}=\frac{\Lambda_{\alpha}^{q} \Lambda^{(q-p)(k-1)}-\Lambda^{-p(k-1)}-\varepsilon \Lambda_{\alpha}^{p}}{2 \varepsilon \Lambda_{\alpha}^{p}} .
$$

The $B_{k, \alpha}$ are bounded away from zero for all $k, \alpha$. First rewrite (B.14), using (2.2) for $\Lambda_{\alpha}$ as

$$
B_{k+1, \alpha}=\frac{\Lambda_{\alpha}^{-p}\left(\Lambda^{(q-p) k}-\Lambda^{-p k}\right)+\varepsilon\left(\Lambda^{(q-p) k}-1\right)}{2 \varepsilon}
$$

Since $\Lambda>1$, it is clear from the form of (B.15) that if $\Lambda_{\alpha}^{-p}$ has nonzero imaginary part or if it is real and positive, $B_{k, \alpha}$ are bounded away from zero for all $k>0$. The only case which requires some more care is when $\Lambda_{\alpha}^{-p}=-b$ for some real $b, b>0$. Since $\Lambda_{\alpha}$ satisfies (2.2), $b=1+O(\varepsilon)$, and (B.15) can be written in this case as

$$
B_{k+1, \alpha}=\frac{(-1+O(\varepsilon)) \Lambda^{(q-p) k}+(1+O(\varepsilon)) \Lambda^{-p k}-\varepsilon}{2 \varepsilon} .
$$

Hence, for sufficiently small $\varepsilon$, the $B_{k, \alpha}$ are strictly decreasing with $k$. Since $B_{1, \alpha}=0$ it follows that the $B_{k, \alpha}$ are indeed bounded away from zero for all $k, \alpha$.

\section{Appendix C: Numerical Error Analysis}

Here we use the linear deviation analysis to estimate the numerical errors in the computation of $u(t), s(r), \delta(t), \delta_{0}, \delta_{1}$, and $c_{1}$. We use a superscript $N$ to denote the numerical values and no superscript to denote the exact values of the various variables. Also, we use $e_{x}, E_{X}$, and $\mathrm{E}_{\mathrm{x}}$, to denote the differences between the exact and computed values of a scalar quantity $x$, a vector $X$, and a matrix $\mathbf{X}$ respectively. For example, $e_{x}=x-x^{N}$.

\section{A. Error Estimate for $u(t)$ and $s(r)$}

It follows from our linear deviation analysis that for sufficiently small initial error

$$
e_{u}(t)=u(t)-u^{N}(t)=\sum_{\alpha} d_{\alpha} v_{\alpha}(t)
$$

and the magnitude of the $d_{\alpha}$ 's is determined by the initial error $e_{u}\left(t_{0}\right)$. Assuming that there are no cancellations in the right-hand side of (C.1) and that $t_{0} \leq 1$, we estimate

$$
\left|d_{\alpha}\right| \sim\left|e_{u}\left(t_{0}\right)\right| t_{0}^{-\operatorname{Re}\left\{x_{\alpha}\right\}}
$$

We further assume that the tangential error may be ignored. Then, for $t \leq 1$ we obtain

$$
\begin{array}{lll}
\left|e_{u}(t)\right| \sim\left|e_{u}\left(t_{0}\right)\right|\left(t / t_{0}\right)^{\rho_{\max },} & t_{0} \ll t, \\
\left|e_{u}(t)\right| \sim\left|e_{u}\left(t_{0}\right)\right|\left(t / t_{0}\right)^{\rho_{\min },} & t_{0} \gg t,
\end{array}
$$


where

$$
\begin{aligned}
& \rho_{\max }=\max _{\alpha \neq 0} \operatorname{Re}\left\{\chi_{\alpha}\right\}, \\
& \rho_{\min }=\min _{\alpha} \operatorname{Re}\left\{\chi_{\alpha}\right\} .
\end{aligned}
$$

The methods we use to compute $\delta(t), \delta_{0}$, and $\delta_{1}$ are constructed to minimize the tangential error, hence the latter assumption is justified for what follows.

Exactly the same considerations for $s(r)$ imply

$$
\begin{array}{lll}
\left|e_{s}(r)\right| \sim\left|e_{s}\left(r_{0}\right)\right|\left(r / r_{0}\right)^{\tilde{\rho}_{\max },} & & r_{0} \ll r, \\
\left|e_{s}(r)\right| \sim\left|e_{s}\left(r_{0}\right)\right|\left(r / r_{0}\right)^{\tilde{\rho}_{\min },} & r_{0} \gg r,
\end{array}
$$

where

$$
\begin{aligned}
& \tilde{\rho}_{\max }=\max _{\alpha \neq 0} \operatorname{Re}\left\{\tilde{\chi}_{\alpha}\right\}, \\
& \tilde{\rho}_{\min }=\min _{\alpha} \operatorname{Re}\left\{\tilde{\chi}_{\alpha}\right\},
\end{aligned}
$$

and to leading order in $\varepsilon$

$$
\begin{aligned}
& \rho_{\max }=\tilde{\rho}_{\max }=\max _{\alpha \neq 0} \cos \left(\frac{2 \pi p \alpha}{q}\right), \\
& \rho_{\min }=\tilde{\rho}_{\min }=\min _{\alpha} \cos \left(\frac{2 \pi p \alpha}{q}\right) .
\end{aligned}
$$

To leading order in $\varepsilon, \rho_{\max } \leq 0$ for $q \leq 4$ and $\rho_{\max }>0$ for $q \geq 5$. Hence, the numerical errors are enhanced more rapidly when $q \geq 5$.

\section{B. Error Estimate for $\delta\left(t_{1}\right)$ and the $\left|c_{\alpha}\right|$ 's-the Real $t_{1}$ Computation}

Recall our method for computing $\delta\left(t_{1}\right)$. First, we determine the value of $t_{1}$ by solving

$$
u\left(t_{1}\right)=u\left(\Lambda^{j} t_{0}\right)=u_{0}(1)
$$

Equation (C.9) is solved numerically using a Newton method to determine the value of $t_{0}$, subject to the constraint that $U\left(t_{0}\right)$ is on the unstable curve. We satisfy this constraint by using the Taylor series of $u$ to evaluate both $u\left(t_{0}\right)$ and its derivative. Once the Newton iterations converge and enough terms in the Taylor expansion are used, we have

$$
\left|e_{u}\left(t_{1}\right)\right|=\left|u\left(\Lambda^{j} t_{0}\right)-u^{N}\left(\Lambda^{j} t_{0}^{N}\right)\right| \sim 10^{-15}
$$

and

$$
\left|e_{u}\left(t_{0}^{N}\right)\right|=\left|u\left(t_{0}^{N}\right)-u^{N}\left(t_{0}^{N}\right)\right| \sim 10^{-15} .
$$

Namely, the error in determining $u\left(t_{1}\right)$ is zero (to machine accuracy) by construction, 
and the numerical error is swept to the determination of $t_{0}$. Indeed, (C.4) gives

$$
\left|e_{u}\left(t_{0}\right)\right|=\left|u\left(t_{0}\right)-u^{N}\left(t_{0}^{N}\right)\right| \sim\left|e_{u}\left(t_{1}\right)\right|\left(\frac{t_{0}}{t_{1}}\right)^{P_{\min }},
$$

leading to

$$
\left|t_{0}^{N}-t_{0}\right| \sim \frac{\left|e_{u}\left(t_{1}\right)\right|\left(t_{0} / t_{1}\right)^{\rho_{\min }}+\left|e_{u}\left(t_{0}^{N}\right)\right|}{\left|u_{t}\left(t_{0}\right)\right|}
$$

For future reference, we substitute in (C.12) the typical numerical values from the computations: $t_{0} \sim O\left(10^{-1}\right), t_{1} \sim O(1), u_{t}\left(t_{0}^{N}\right) \sim t_{0}^{N} \sim t_{0}, \rho_{\min } \geq-1$, and get

$$
\left|e_{t_{0}}\right| \equiv\left|t_{0}^{N}-t_{0}\right| \lesssim 10^{-13} \text {. }
$$

To proceed, we calculate $s\left(r\left(t_{1}\right)\right)$, where $r\left(t_{1}\right)$ is defined as the $r$ value for which $c_{0}$ vanishes, by solving

$$
c_{0}=\mathbf{V}^{-1}\left(t_{1}\right)\left(U\left(t_{1}\right)-S\left(\Gamma^{j} r_{0}\right)\right)(1)=0
$$

for $r_{0}=\Gamma^{-j}\left(r\left(t_{1}\right)\right) . \mathbf{V}^{-1}\left(t_{1}\right)$ is the inverse of the matrix $\mathbf{V}\left(t_{1}\right)$, the matrix which is spanned column-wise by the $V_{\alpha}$ 's. This method of computing $S\left(r\left(t_{1}\right)\right)$ guarantees that the tangential error is indeed zero to machine accuracy; hence,

$$
\left|e_{s}\left(r\left(t_{1}\right)\right)\right| \sim\left|e_{s}\left(r_{0}\right)\right|\left(r\left(t_{1}\right) / r_{0}\right)^{\rho_{\max }} \sim \begin{cases}10^{-15}, & q \leq 4 \\ 10^{-14}, & q \geq 5,\end{cases}
$$

and the numerical error in the computation of $\delta\left(t_{1}\right)=\left\|U\left(t_{1}\right)-S\left(r\left(t_{1}\right)\right)\right\|$ is

$$
\left|e_{\delta\left(t_{1}\right)}\right| \leq\left\|E_{U}\left(t_{1}\right)\right\|+\left\|E_{S}\left(r\left(t_{1}\right)\right)\right\| \sim \begin{cases}10^{-15}, & q \leq 4, \\ 10^{-14}, & q \geq 5 .\end{cases}
$$

The above estimates demonstrate that when $\delta\left(t_{1}\right) \sim 10^{-15}$, this method of computations fails. For the quadratic map with $q=3$, we cannot use this method for $\varepsilon \leq 0.4$.

Finally, we compute the $c_{\alpha}$ 's using

$$
C^{N}=\mathbf{V}^{-1 N}\left(t_{1}\right)\left[U^{N}\left(t_{1}\right)-S^{N}\left(r\left(t_{1}\right)\right)\right]
$$

where

$$
C_{j}= \begin{cases}c_{\alpha}, & \text { for } \alpha \geq 0 \\ c_{[q / 2]-\alpha}, & \text { for } \alpha<0\end{cases}
$$

and the $V_{\alpha}$ 's are ordered accordingly. There are two sources of error in this computation of the $c_{\alpha}$ 's; the first is the numerical error in evaluating the right-hand side of (C. 17) and the second is the negligence of the nonlinear interaction of the $V_{\alpha}$ 's. Including these terms formally, we write

$$
[S(r(t))-U(t)]_{j}=\sum_{\alpha} \mathbf{V}_{j, \alpha}(t)\left(c_{\alpha}+\sum_{\beta} d_{\alpha \beta}(t) \mathbf{V}_{j, \beta}(t)\right)+O\left(\left|c_{1} V_{1}(t)\right|^{3}\right)
$$


where $d_{\alpha \beta}(t)$ can be found from the general solution to the equations for the quadratic terms. Since the error estimates are performed for $|t|=O(1)$, we assume that $d_{\alpha \beta}(t) \approx d_{\alpha \beta}(1)$ in this region, and

$$
d_{\alpha \beta}(1)=O\left(c_{\alpha} c_{\beta}\right)
$$

Subtracting

$$
\left[U^{N}(t)-S^{N}(r(t))\right]_{j}=\sum_{\alpha} \mathbf{v}_{j, \alpha}^{N}(t) c_{\alpha}^{N}
$$

from the exact difference, we obtain

$\left[E_{U}-E_{S}\right](j)=\left(\mathbf{V} E_{C}\right)_{j}+\left(\mathbf{E}_{\mathrm{v}} C\right)_{j}+\left(\mathbf{E}_{\mathbf{v}} E_{C}\right)_{j}+\sum_{\alpha, \beta} d_{\alpha \beta} \mathbf{V}_{j, \alpha}(t) \mathbf{V}_{j, \beta}(t)+O\left(\left\|c_{1} V_{1}(t)\right\|^{3}\right)$

Using (4.23) to find $\mathrm{V}^{-1}$ to leading order in $\varepsilon$, we find

$$
\begin{aligned}
E_{c_{\gamma}}= & \mathbf{V}^{-1}\left[E_{U}-E_{S}-\mathbf{E}_{\mathbf{v}} C-\mathbf{E}_{\mathbf{v}} E_{C}\right]_{\gamma} \\
& +\sum_{\alpha, \beta} d_{\alpha \beta} t^{\chi_{\alpha}+\chi_{\beta}-\chi_{\gamma}} \sum_{j} \Lambda^{j\left(\chi_{\alpha}+\chi_{\beta}-\chi_{\gamma}\right)}(1+O(\varepsilon))+O\left(\left\|c_{1} V_{1}(t)\right\|^{3}\right) \\
= & \mathbf{V}^{-1}\left[E_{U}-E_{S}-\mathbf{E}_{\mathrm{v}} C-\mathbf{E}_{\mathbf{v}} E_{C}\right]_{\gamma} \\
& +\sum_{\alpha, \beta} d_{\alpha \beta} F(\alpha, \beta, \gamma) t^{\chi_{\alpha}+\chi_{\beta}-\chi_{\gamma}}+O\left(\left\|c_{1} V_{1}(t)\right\|^{3}\right)
\end{aligned}
$$

where

$$
F(\alpha, \beta, \gamma)= \begin{cases}q+O(\varepsilon), & \alpha+\beta-\gamma=0(\bmod q) \\ O(\varepsilon), & \text { otherwise. }\end{cases}
$$

Now, we estimate the right-hand side of (C.18). First, we estimate $\mathbf{E}_{\mathbf{v}}$. Let $t_{1}=\Lambda^{k} t_{0}$,

$$
\begin{aligned}
\left|\mathbf{E}_{\mathrm{v}_{j, \alpha}}\right| & =\left|v_{\alpha}\left(\Lambda^{j+k} t_{0}\right)-v_{\alpha}^{N}\left(\Lambda^{j+k} t_{0}^{N}\right)\right| \\
& \sim \mid \Lambda_{\alpha}^{k+j}\left\{t_{0}^{\chi_{\alpha}} \tilde{v}_{\alpha}\left(\Lambda^{j} t_{1}\right)-\left(t_{0}-e_{t_{0}}\right)^{\chi_{\alpha}}\left(\tilde{v}_{\alpha}\left(\Lambda^{j} t_{1}\right)-e_{\tilde{v}_{\alpha}}\left(\Lambda^{j} t_{1}\right)\right.\right. \\
& \left.\left.\quad-\tilde{v}_{\alpha}\left(\Lambda^{j} t_{1}\right) \Lambda^{j} t_{1} e_{t_{0}} / t_{0}\right)\right\} \mid \\
& \sim\left|t_{0}^{\chi_{\alpha}} \Lambda_{\alpha}^{k+j}\left[e_{\tilde{v}_{\alpha}}\left(\Lambda^{j} t_{1}\right)+\frac{e_{t_{0}}}{t_{0}}\left(\chi_{\alpha} \tilde{v}_{\alpha}\left(\Lambda^{j} t_{1}\right)+\tilde{v}_{\alpha t}\left(\Lambda^{j} t_{1}\right) \Lambda^{j} t_{1}\right)\right]\right| \\
& \sim\left|v_{\alpha}\left(\Lambda^{j} t_{1}\right)\left\{\frac{e_{\tilde{v}_{\alpha}}\left(\Lambda^{j} t_{1}\right)}{\tilde{v}_{\alpha}\left(\Lambda^{j} t_{1}\right)}+\frac{e_{t_{0}}}{t_{0}}\left[\chi_{\alpha}+\Omega^{\alpha p}\left(-1+g^{\prime}\left(u\left(\Lambda^{j} t_{1}\right)\right) / g^{\prime}(0)\right)\right]\right\}\right| \\
& \lesssim\left|v_{\alpha}\left(\Lambda^{j} t_{1}\right)\right|\left(K \frac{\left|e_{t_{0}}\right|}{t_{0}}+\left|\frac{e_{\tilde{v}_{\alpha}}\left(\Lambda^{j} t_{1}\right)}{\tilde{v}_{\alpha}\left(\Lambda^{j} t_{1}\right)}\right|\right)
\end{aligned}
$$

where $K=O(1)$ to leading order. In (C.19) we have used (3.16a) to estimate the derivative of $\tilde{v}_{\alpha}(t)$. Also, one can neglect the term $e_{\tilde{v}_{\alpha}}\left(\Lambda^{j} t_{1}\right) / \tilde{v}_{\alpha}\left(\Lambda^{j} t_{1}\right)$ since the linearity of $(3.11 b)$ and the slow growth of all the $\tilde{v}$ 's with $t$ imply 


$$
\left|e_{\tilde{v}_{\alpha}\left(\Lambda^{i} t_{1}\right)}\right| \sim\left|e_{\tilde{v}_{\alpha}\left(t_{0}\right)}\right| \sim 10^{-15} \ll \frac{\left|e_{t_{0}}\right|}{t_{0}}\left|\tilde{v}_{\alpha}\left(\Lambda^{j} t_{1}\right)\right| .
$$

Equations (C.19) and (C.20) imply that

$$
\mathbf{E}_{\mathbf{v}} \sim K \frac{e_{t_{0}}}{t_{0}} \mathbf{V}
$$

hence,

$$
\left(\mathbf{V}^{-1} \mathbf{E}_{\mathrm{v}} E_{C}\right)_{\gamma} \sim K \frac{e_{t_{0}}}{t_{0}} E_{c_{\gamma}}
$$

and therefore the latter term contributes only to higher-order corrections of $E_{c_{\gamma}}$. Using (C.18)-(C.21), we conclude that

$\left|E_{c_{\gamma}}\right| \leq\left|\mathbf{V}^{-1}\left[E_{U}-E_{S}\right]\right|+K \frac{\left|e_{t_{0}}\right|}{t_{0}}\left|c_{\gamma}\right|+\left|\sum_{\alpha, \beta} d_{\alpha \beta} F(\alpha, \beta, \gamma) t^{x_{\alpha}+\chi_{\beta}-x_{\gamma}}\right|+O\left(\left\|c_{1} V_{1}(t)\right\|^{3}\right)$.

For real $t$ of order one and $\varepsilon \ll 1,\|\mathbf{V}\|,\left\|\mathbf{V}^{-1}\right\|=O(1)$, and $\left|e_{t_{0}} c_{\gamma}\right| / t_{0} \ll\left\|E_{U}\right\|$; therefore,

$$
\begin{aligned}
\left|E_{c_{\gamma}}\right| & \lesssim\left\|E_{U}\right\|+\left\|E_{S}\right\|+\max _{\alpha, \beta}\left|F(\alpha, \beta, \gamma) d_{\alpha \beta}\right| \\
& \sim \max \left(\left\|E_{U}\right\|,\left\|E_{S}\right\|, K_{q, \gamma}\left|c_{1}\right|^{2}\right)
\end{aligned}
$$

where $K_{q, \gamma}$ has at most a power law dependence on $\varepsilon$, and the dependence of all the quantities on $t$ is suppressed.

For the quadratic map with $q=3$ and $\varepsilon=0.75,\left|c_{1}\right| \sim 5 \times 10^{-9}$; hence, according to (C.23) the error in $\left|c_{1}\right|$ is estimated to vanish to machine accuracy. We computed $\left|c_{1}\right|$ for a range of $t$ values of order 1 and verified the oscillations in $t$ are of the order of $10^{-16}$. A similar calculation for the case $g(x)=x\left(1-x^{2}\right)$, with $q=5, p=2$, and $\varepsilon=0.5$ gives oscillations of the order $10^{-13}$, shown in Figure 8. Indeed, in this case $\left|c_{1}\right| \sim 3 \times 10^{-7}$, and (C.23) predicts oscillations of the order $10^{-13}$. The oscillations of $\left|c_{1}\right|$ in $t$ are associated with the oscillating $v_{1}(t)$ term, contained in the quadratic corrections. The little "bumps" which are superimposed on this mode (see Figure 8) may be associated with the algebraic corrections in $\varepsilon$, and the general trend of decreasing error with $t$ may be related to the $t$-dependence of $d_{1,1}$.

The error estimate of $c_{\gamma}$ demonstrates two limitations on the computation. First, that one cannot compute $c_{\alpha}$ for $|\alpha|>1$. Second, that the computation of $c_{ \pm 1}$ is limited to sufficiently large $\varepsilon$ values for which $\left|c_{ \pm 1}(\varepsilon)\right|>10^{-15}$. The first limitation is not crucial since these coefficients are insignificant for evaluating the splitting distance. We overcome the second limitation by performing the same calculation in the matching region of the complex $t$ plane, where all quantities are exponentially enhanced.

\section{Error Estimate for $\delta\left(t_{c}\right)$ and $c_{1}-$ Complex $t$ calculation}

Recall our procedure for computing $\delta\left(t_{c}\right)$ and $c_{ \pm 1}$ using complex $t$. First, we fix the phase of $t$ to be $\left(\phi_{s}-\varepsilon \phi_{1}\right)$ and find the value of $\left|t_{0}\right|$ for which $\Lambda^{j} t_{0}$ is closest to the singularity $\left(\phi_{s}>0\right.$ when $\phi_{1}<0$ and vice versa). We initialize $u$ and the $\tilde{v}_{\alpha}$ 's at this 
value of $t_{0}$ and advance them $j$ times. Since the phase of $t$ is fixed (namely $t_{c} / t_{0}$ is a real number), the estimates for the error in the calculation of $U(t), t_{0}, S(r), \delta\left(t_{c}\right)$, and $\tilde{v}_{\alpha}\left(t_{c}\right)$ are given by (C.10), (C.13), (C.15), (C.16), and (C.20) respectively. The main difference between the real and complex calculations is the treatment of the ${ }_{t}^{X_{\alpha}}$ terms which appear in $\mathbf{V}$ and their contribution to $\left\|E_{C}\right\|$. These terms may be exponentially small (or large) for complex $t$ even when $|t|=O(1)$. We avoid the problem of computation with small (or large) numbers by normalization. We replace (C.17) by

$$
C_{\text {norm }}=\mathbf{V}_{\text {norm }}^{-1}(t)[S(r(t))-U(t)]
$$

where

$$
\begin{aligned}
V_{j, \alpha \text { norm }}(t) & =t_{\text {norm }}^{-\chi_{\alpha}} V_{\alpha}\left(\Lambda^{j-1} t\right)=\left(t / t_{\text {norm }}\right)^{\chi_{\alpha}} \tilde{V}_{\alpha}\left(\Lambda^{j-1} t\right) \\
t_{\text {norm }} & =t_{c}\left(\varepsilon_{\min }\right)=\left|t_{c}\left(\varepsilon_{\min }\right)\right| e^{i\left(\phi_{s}-\varepsilon_{\min } \phi_{1}\right)}
\end{aligned}
$$

and $\varepsilon_{\min }$ is the smallest value of $\varepsilon$ for which the calculation is performed. This normalization makes the entries of the matrix $V_{\text {norm }}$ and its inverse to be of order 1 . Finally, we find $t_{s}^{\chi_{\alpha}} c_{\alpha}$ (an order-1 quantity) from

$$
t_{s}^{\chi_{\alpha}} c_{\alpha}=c_{\alpha \text { norm }}\left(t_{s} / t_{\text {norm }}\right)^{\chi_{\alpha}}=c_{\alpha \text { norm }} e^{i \phi_{1} \chi_{\alpha} \varepsilon_{\min }}\left|t_{s} / t_{c}\left(\varepsilon_{\min }\right)\right|^{\chi_{\alpha}},
$$

avoiding the explicit use of exponentially small quantities. For the error analysis itself, it is easy to show that the normalization factor drops out; hence, for simplicity of notation we do the analysis for the nonnormalized quantities.

To find the error in $t_{s}^{\chi_{\alpha}} c_{\alpha}$, we multiply the right-hand side of (C.22) by $t_{s}^{X_{\alpha}}$, and evaluate it at $t=t_{c}$

$$
\begin{aligned}
& \left|E_{t_{s}^{\gamma_{\gamma}} c_{\gamma}}\right| \lesssim\left|t_{s}^{\chi_{\gamma}}\right| \sum_{j}\left|\mathbf{V}_{\gamma, j}^{-1}\left(t_{c}\right)\right|\left[\left|E_{U j}\right|+\left|E_{S j}\right|\right]+K \frac{\left|e_{t_{0}}\right|}{\left|t_{0}\right|}\left|t_{s}^{x_{\gamma}} c_{\gamma}\right| \\
& +\left|\left(t_{s} / t_{c}\right)^{x_{\gamma}}\right|\left(\left|\sum_{\alpha, \beta} d_{\alpha \beta} F(\alpha, \beta, \gamma) t_{c}^{\alpha_{\alpha}+\chi_{\beta}}\right|+O\left(\left\|c_{1} V_{1}(t)\right\|^{\beta}\right)\right) \\
& \leq\left|\left(t_{s} / t_{c}\right)^{\chi_{\gamma}}\right|\left[|| E_{U}\|+\| E_{S} \|\right]+K \frac{\left|e_{t_{0}}\right|}{\left|t_{0}\right|}\left|t_{s}^{\chi_{\gamma}} c_{\gamma}\right| \\
& +\tilde{K}\left|\left(t_{s} / t_{c}\right)^{\chi_{\gamma}}\right| \max _{\alpha}\left|c_{\alpha}^{2} t_{c}^{2 \chi_{\alpha}}\right|+\left|\left(t_{s} / t_{c}\right)^{\chi_{\gamma}}\right| O\left(\left\|c_{1} V_{1}(t)\right\|^{3}\right) \\
& \lesssim \exp \left(2 \pi\left|\phi_{1} \gamma\right|\right)\left[\left\|E_{U}\right\|+\left\|E_{S}\right\|\right]+K \frac{\left|e_{t_{0}}\right|}{\left|t_{0}\right|}\left|t_{s}^{x_{\gamma}} c_{\gamma}\right| \\
& +\tilde{K} \exp \left(-2 \pi\left|\phi_{1}\right|(2-|\gamma|)\right)+\exp \left(2 \pi\left|\phi_{1} \gamma\right|\right) O\left(\left\|c_{1} V_{1}(t)\right\|^{3}\right)
\end{aligned}
$$

where $K, \tilde{K}$ are constants to leading order in $\varepsilon$. The higher-order correction term is of the order $\exp \left(-6 \pi \phi_{1}\right)$ and hence negligible. Also, to leading order, when $\gamma \phi_{1}<0$, $t_{s}^{\chi_{y}} c_{\gamma}=O(1)$, and the relative error in this case is given by

$$
\begin{aligned}
\left|\frac{E_{t_{s} x_{\gamma}} c_{\gamma}}{t_{s}^{x_{\gamma}} c_{\gamma}}\right| & \lesssim \max \left(\exp \left(2 \pi\left|\phi_{1} \gamma\right|\right)\left[\left\|E_{U}\right\|+\left\|E_{S}\right\|\right], K\left|\frac{e_{t_{0}}}{t_{0}}\right|, \tilde{K} \exp \left(-2 \pi\left|\phi_{1}\right|(2-|\gamma|)\right)\right) \\
& \left.\lesssim \max \left(\exp \left(2 \pi\left|\phi_{1} \gamma\right|\right)\left[\mid E_{U}\|+\| E_{S} \|\right], \tilde{K} \exp \left(-2 \pi\left|\phi_{1}\right|(2-|\gamma|)\right)\right) . \quad \text { (C. } 27\right)
\end{aligned}
$$


If $\gamma \phi_{1}>0,\left|t_{s}^{\chi_{\gamma}} c_{\gamma}\right| \sim\left|c_{\gamma}\right|^{2}$ and the relative error becomes large as $\varepsilon$ is decreased, namely, one has to choose the correct phase in order to obtain $c_{ \pm 1}$. Also, (C.27) demonstrates that one cannot find $c_{\gamma}$ for $|\gamma|>1$.

In the computation, we search for an interval of $\left|\phi_{1}\right|$ for which $c\left(\phi_{1}\right)$ is roughly independent of $\phi_{1}$. We define $\left|\phi_{c}\right|$ to be the value for which $c_{1}\left(\phi_{c}\right)$ takes the average value in this interval. In Figure A.1, we present a plot of $\left|c_{-1}^{N}\left(\phi_{1}\right)-c_{-1}\left(\phi_{c}\right)\right|$, confirming the exponential dependence of the error on $\phi_{1}$. It follows from (C.15) and (C.27) that the minimal error in $c_{1}$ is given by

$$
\frac{\left|e_{c_{ \pm 1}}\right|}{\left|c_{ \pm 1}\right|} \sim \sqrt{\left\|E_{U}\right\| / \tilde{K}}
$$

and that this error is realized at

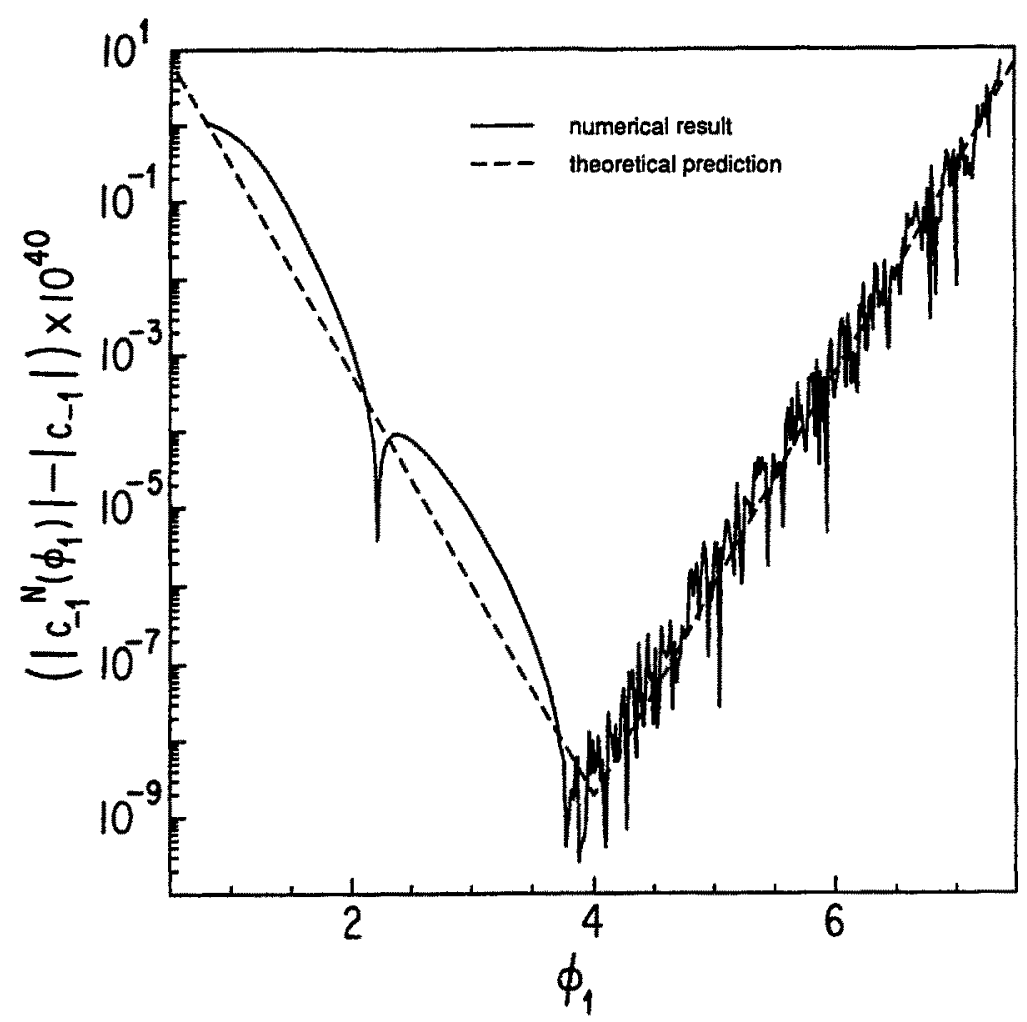

Fig. A.1. The dependence of the numerical value of $\left|c_{ \pm 1}\right|$ on the phase of $t$. We plot $\left|\left(c_{-1}^{N}\left(\phi_{1}\right)|-| c_{-1} \mid\right)\right|$ for the case $g(x)=x\left(1-x^{2}\right), q=5$, and $p=2$ with $\varepsilon=0.1$, where $t=\left|t_{c}\right| \exp \left(i\left(\phi_{s}-\varepsilon \phi_{1}\right)\right)$, and $\left|c_{-1}\right|=5.1974207 \times 10^{-40}$. We also plot the theoretical prediction for the error (see (C.27)), where $\tilde{K}$ is determined to obtain the best fit. A least-square fit for $\ln \left|\left(\left|c_{-1}^{N}\left(\phi_{1}\right)\right|-\left|c\left(\phi_{c}\right)\right|\right)\right|$ gives a slope of $-6.2(6.0)$ for $\left.\phi_{1}<\phi_{c}\left(\phi_{1}\right\rangle \phi_{c}\right)$, whereas our error analysis gives a slope of $-2 \pi(2 \pi)$. 
Table A.1 Numerical values of $\left|c_{-1}\right|$ for various $g(x)$ 's.

\begin{tabular}{|c|c|c|c|c|c|}
\hline Function, $g(x)$ & $m$ or $a$ & $q$ & $p$ & $\varepsilon$ & $\left|c_{-1}\right|$ \\
\hline \multirow{6}{*}{$x\left(1-x^{m}\right)$} & \multirow{2}{*}{1} & 3 & 2 & 0.100 & $4.0630759 \times 10^{-81}$ \\
\hline & & 5 & 3 & 0.100 & $6.498439 \times 10^{-81}$ \\
\hline & \multirow[t]{2}{*}{2} & 3 & 2 & $\begin{array}{l}0.005 \\
0.010 \\
0.050 \\
0.100 \\
0.300 \\
0.400 \\
0.500 \\
0.600 \\
0.700\end{array}$ & $\begin{array}{r}8.262491 \times 10^{-854} \\
1.4798233 \times 10^{-425} \\
1.5327448 \times 10^{-83} \\
4.496933 \times 10^{-20} \\
3.6488399 \times 10^{-13} \\
8.846798 \times 10^{-10} \\
8.70057 \times 10^{-8} \\
1.74400 \times 10^{-6} \\
1.41825 \times 10^{-5}\end{array}$ \\
\hline & & 5 & 3 & 0.100 & $9.377381 \times 10^{-41}$ \\
\hline & \multirow{2}{*}{3} & 3 & 2 & 0.100 & $8.345954 \times 10^{-28}$ \\
\hline & & 5 & 3 & 0.100 & $1.8377573 \times 10^{-27}$ \\
\hline \multirow{3}{*}{$x(1-x)(1+a x)$} & \multirow{2}{*}{$-\frac{1}{2}$} & 3 & 2 & 0.100 & $1.982035 \times 10^{-82}$ \\
\hline & & 5 & 3 & 0.100 & $1.525768 \times 10^{-82}$ \\
\hline & $\frac{1}{2}$ & 3 & 2 & 0.100 & $4.227849 \times 10^{-55}$ \\
\hline \multirow{2}{*}{$\frac{\sin \pi x}{\pi}$} & & 3 & 2 & 0.100 & $3.937723 \times 10^{-42}$ \\
\hline & & 5 & 3 & 0.100 & $4.462319 \times 10^{-42}$ \\
\hline
\end{tabular}

$$
\phi_{m}=\frac{\ln \left\|E_{U}\right\|-\ln \tilde{K}}{4 \pi} \approx 2.748-\frac{\ln \tilde{K}}{4 \pi} .
$$

Numerically, we find that $\phi_{c} \approx 3.4$, and that the relative error is approximately $10^{-7}$. These results are consistent with the above estimates when $\tilde{K} \sim 80, \phi_{m} \sim 3.1$ and one examines an interval of length 0.7 in $\phi_{1}$. In Table A.1, we quote some numerical values of $\left|c_{-1}\right|$ for various $\varepsilon$ values and for various $g(x)$ 's. We present six to seven significant digits, the number of digits which are identical for different $\phi_{1}$ values in an interval near $\phi_{c}$.

\section{Error Estimation for $\delta_{0}$ and $\delta_{1}$}

Recall that the definitions of $\delta_{0}$ and $\delta_{1}$ are

$$
\begin{aligned}
& \delta_{0}=\min _{r}\|S(r)-0\|, \\
& \delta_{1}=\min _{t}\|U(t)-1\| .
\end{aligned}
$$


Hence, the only source of error in the computation of $\delta_{0}$ and $\delta_{1}$ is the accumulating error in the computation of $S(r)$ and $U(t)$, respectively. Also, since $\delta_{0}$ and $\delta_{1}$ are found via minimization on the parameters $r$ and $t$, respectively, it is clear that the errors in the tangential direction are irrelevant. In section $A$ of this appendix, we established that for $\bar{t}=O(1)$ (respectively $\bar{r}=O(1)$ ), $e_{u}(\bar{t})$ and $e_{s}(\bar{r})$ are given by (C.3) and (C.6), respectively. However, from our analysis, we know that the minima in (C.30) are attained for $r_{\min } \gg 1$ and $t_{\min } \gg 1$, respectively. We use the result that $U(t)$ is very close to $S(r(t))$ for $\bar{t} \leq t \leq t_{\min }$ to approximate the growth rate of $e_{u}(t)$ for $t \gg 1$ by the linearized modes about $S(r(t))$. Namely, we use (C.3) and (C.8) to obtain

$$
e_{u}\left(t_{\min }\right)=e_{u}\left(t_{0}\right)\left(\frac{\bar{t}}{t_{0}}\right)^{\rho_{\max }}\left(\frac{r\left(t_{\min }\right)}{r(\bar{t})}\right)^{\tilde{\rho}_{\min }}
$$

and, similarly,

$$
e_{s}\left(r_{\min }\right)=e_{s}\left(r_{0}\right)\left(\frac{\bar{r}}{r_{0}}\right)^{\tilde{\rho}_{\max }}\left(\frac{t\left(r_{\min }\right)}{t(\bar{r})}\right)^{\rho_{\min }}
$$

Setting $\bar{t}=\bar{r}=1$, we get

$$
\begin{aligned}
& e_{u}\left(t_{\min }\right) \sim e_{u}\left(t_{0}\right) t_{0}^{-\rho_{\max }} r\left(t_{\min }\right)^{\bar{\rho}_{\min }}, \\
& e_{s}\left(r_{\min }\right) \sim e_{s}\left(r_{0}\right) r_{0}^{-\bar{\rho}_{\max }} t\left(r_{\min }\right)^{\rho_{\min }} .
\end{aligned}
$$

We use (4.26) to estimate the value of $r\left(t_{\min }\right)$ and $t\left(r_{\min }\right)$ in (C.32) to obtain

$$
\begin{aligned}
& e_{u}\left(t_{\min }\right) \sim e_{u}\left(t_{0}\right) t_{0}^{-\rho_{\max }}\left(\tilde{K}_{\tilde{\alpha}}\left|\tilde{c}_{1}\right|^{\eta_{1}}\right)^{\tilde{\rho}_{\min }}, \\
& e_{s}\left(r_{\min }\right) \sim e_{s}\left(r_{0}\right) r_{0}^{-\tilde{\rho}_{\max }}\left(K_{\hat{\alpha}}\left|c_{1}\right|^{\eta_{\infty}}\right)^{\rho_{\min }},
\end{aligned}
$$

where $\tilde{\alpha}$ and $\hat{\alpha}$ are the $\alpha$ values which minimize $\tilde{\rho}_{\min }$ and $\rho_{\min }$, respectively. Finally, the relative errors are given by

$$
\begin{aligned}
& \frac{e_{u}\left(t_{\min }\right)}{\delta_{1}} \sim e_{u}\left(t_{0}\right) t_{0}^{-\rho_{\max }}\left(\tilde{K}_{\tilde{\alpha}}\left|\tilde{c}_{1}\right|^{\eta_{1}}\right)^{\tilde{p}_{\min }-1}, \\
& \frac{e_{s}\left(r_{\min }\right)}{\delta_{0}} \sim e_{s}\left(r_{0}\right) r_{0}^{-\tilde{p}_{\max }}\left(K_{\hat{\alpha}}\left|c_{1}\right|^{\eta_{0}}\right)^{\rho_{\min }-1} .
\end{aligned}
$$

The $\varepsilon$ values for which the relative errors are of order 1 define the minimal values of $\varepsilon$, denoted by $\varepsilon_{0}$ and $\varepsilon_{1}$, for which the computations of $\delta_{0}$ and $\delta_{1}$ are meaningful. We use the analysis to deduce the $\varepsilon$ dependence of the relative error, and we use the numerics to get the prefactor of $\left|c_{1}\right|$. The prefactor of $\left|\tilde{c}_{1}\right|$ is found from (4.28). For example, when $g(x)=x(1-x), q=3$, and $p=2$, (C.31) gives $\varepsilon_{0}=\varepsilon_{1}=0.437$. Numerically, our method converges for $\varepsilon \geq 0.43$ in the two cases. In Table A.2, we present the theoretical and numerical $\varepsilon_{0}$ and $\varepsilon_{1}$. For $q \leq 4$, the theoretical and numerical values agree to the first significant digit. When $q \geq 5, \varepsilon_{0}$ and $\varepsilon_{1}$ are large and the corrections from the higher-order terms are significant. 
Table A.2 Theoretical and numerical values of $\varepsilon_{0}$ and $\varepsilon_{1}$ for $g(x)=x(1-x)$.

\begin{tabular}{|c|c|c|c|c|c|}
\hline \multicolumn{2}{|c|}{} & \multicolumn{2}{|c|}{ theoretical } & \multicolumn{2}{c|}{ numerical } \\
\hline$q$ & $p$ & $\varepsilon_{0}$ & $\varepsilon_{1}$ & $\varepsilon_{0}$ & $\varepsilon_{1}$ \\
\hline 3 & 2 & 0.437 & 0.437 & 0.43 & 0.43 \\
\hline 4 & 1 & 0.740 & 0.535 & 0.71 & 0.59 \\
\hline 5 & 1 & 0.739 & 0.739 & 0.73 & 0.63 \\
\hline
\end{tabular}

\section{Appendix D: Toward Rigorous Results}

Here we set up a framework which may lead to a theoretical justification of our results. We divide this appendix into two parts. In the first part we outline a proof of the exponential smallness of the distance function; here we relax the sharp estimate of $\beta$. In the second part we formulate a series of steps, which, if proven, wili constitute a proof of the main results of this paper, namely, the sharp estimates of $\beta$ and $\gamma$ in (1.9).

\section{A. The Distance Function Is Exponentially Small in $\varepsilon$}

In this part we give supporting evidence to the following claim.

Claim D.1. Given $a \phi, 0<\phi<\phi_{s}$, and an entire function $g(x)$ which is positive on the open interval $(0,1)$ and satisfies $(1.3)$ and $(1.4)$, there exists an $\varepsilon_{m}=\varepsilon_{m}(\phi)$ and an analytic function $r(t)$ such that for $0<\varepsilon<\varepsilon_{m}$ and real $t$ of order 1

$$
\delta(t)=\|\Delta(t)\|=\|S(r(t))-U(t)\| \leq B(\varepsilon, \phi) \xi^{\phi / \phi_{s}},
$$

where $B(\varepsilon, \phi) \rightarrow 0$ as $\varepsilon \rightarrow 0^{+}$, and $\xi$ is the small parameter defined by (4.4).

Supporting Evidence (SE). Define the region $R_{\phi}$ in the $\ln (t)$ complex plane as

$$
R_{\phi}=\{t \mid-\phi<\operatorname{Im}\{\ln (\mathrm{t})\}<\phi, \quad \mathrm{a}<\operatorname{Re}\{\ln (\mathrm{t})\}<\mathrm{A}\},
$$

where $0<a<\Lambda_{\varepsilon_{m}(\phi)}^{-2} A<\infty$. We estimate the magnitude of $\Delta(t)$ in $R_{\phi}$ and conclude its exponential smallness on the real axis. For clarity, we first state the essential lemmas and claims which constitute the proof of Claim D.1, and then outline their supporting evidence.

Lemma 1. In $R_{\phi}$, the vector-valued functions $U(t), S(t)$, and $\tilde{V}_{\alpha}(t)$ are analytic (hence bounded) for all $0 \leq \varepsilon<\varepsilon_{m}$.

We denote the uniform bound of a function $f(t)$, valid for all $t \in R_{\phi}$ and $0 \leq \varepsilon<$ $\varepsilon_{m}(\phi), b y \hat{f}(\phi)$. 
Claim 1. In $R_{\phi}$, all the above-mentioned functions have a unique, analytic limit as $\varepsilon \rightarrow 0^{+}$.

We denote the limit $\varepsilon \rightarrow 0^{+}$of a function $f(t)$ by $f_{0}(t)$.

Claim 2. The matrix $V$ is nonsingular in $R_{\phi}$, and its inverse $W$ is of the form

$$
W_{\alpha, j}(t)=\left(\Lambda^{j} t\right)^{-\chi_{\alpha}} \tilde{W}_{\alpha, j}(t) .
$$

The functions $\tilde{W}_{\alpha, j}(t)$ are analytic (and bounded) for all $0 \leq \varepsilon<\varepsilon_{m}$.

Claim 3. There exists a function $r(t)$, analytic in $R_{\phi}$, such that $r(\Lambda t)=r(t) / \Lambda$ and $\delta(\varepsilon, \phi) \rightarrow 0$ as $\varepsilon \rightarrow 0^{+}$, where $\delta(\varepsilon, \phi)$ denotes the upper bound of $\delta(t)$ in $R_{\phi}$.

Corollary 1. The coefficients $z_{\alpha}(t)$

$$
z_{\alpha}(t)=(\mathbf{W} \Delta(t))_{\alpha}
$$

are analytic in $R_{\phi}$.

Corollary 2. Let $\operatorname{Im}\{\ln (\mathrm{t})\}=\theta$. Then, for $t \in R_{\phi}$,

$$
\begin{aligned}
&\left|v_{\alpha}(t)\right| \leq \exp (-2 \pi \theta \alpha / q \ln \Lambda) \hat{\tilde{v}}(\phi), \\
&\left|w_{\alpha}(t)\right| \leq \exp (2 \pi \theta \alpha / q \ln \Lambda) \hat{\tilde{w}}(\phi) .
\end{aligned}
$$

Corollary 3. In $R_{\phi}$,

$$
\left|z_{\alpha}(t)\right| \leq \xi^{-\alpha \theta / \phi_{s}} \delta(\varepsilon, \phi) \hat{\tilde{w}}(\phi) .
$$

Claim 4. There exist constants $c_{\alpha, 0}$ such that

$$
\begin{aligned}
z_{\alpha}(t)= & c_{\alpha, 0}+e_{\alpha}(t), \\
\left|c_{\alpha, 0}\right| \leq & \xi^{|\alpha| \phi / \phi_{s}} \delta(\varepsilon, \phi) \hat{\hat{w}}(\phi), \\
\left|e_{\alpha}(t)\right| \leq & A E_{\alpha} \xi^{|\alpha| \phi / \phi_{s}} \delta(\varepsilon, \phi) \hat{\tilde{w}}(\phi), \\
E_{\alpha}= & \min \left\{\xi^{(\mp q \theta+\phi(q-2|\alpha|)) / \phi_{s}}, \xi^{q(\phi \pm \theta) / \phi_{s}},\right. \\
& \left.\varepsilon^{-1} \delta(\varepsilon, \phi) \hat{\tilde{w}}(\phi)\left(1+\xi^{[(2-|\alpha|) \phi-(2|\theta|+\alpha \theta)] / \phi_{s}}+\xi^{(\mp q \theta+\phi(q-2|\alpha|)) / \phi_{s}}\right)\right\}
\end{aligned}
$$

where the upper (lower) sign stands for $\alpha>0(\alpha<0)$.

Conjecture 1. Generically, in the space of entire mappings $g(x)$ of the form considered in this paper, the constants $c_{\alpha, 0}$ attain the value of the upper bound of $(D .8)$.

To obtain the order-of-magnitude estimates, we assume that the mappings we consider have this property, namely, we assume

$$
\left|c_{\alpha, 0}\right| \approx \xi^{|\alpha| \phi / \phi_{s}} \delta(\varepsilon, \phi) \hat{\tilde{w}}(\phi) .
$$

Then, it follows from (D.7), (D.9), and (D.10) that for real $t$, the order of magnitude of $z_{\alpha}(t)$ is given by $\left.\left|c_{1,0}\right|\right|^{|\alpha|}$ for $0<|\alpha| \leq 2$, whereas for $|\alpha|>2$ the nonlinear 
interaction of the $V_{ \pm 1}(t)$ modes can dominate. Therefore, using (D.4) (D.5), (D.7), (D.9), and (D.10) we obtain (D.1) with

$$
B(\varepsilon, \phi)=\delta(\varepsilon, \phi) \hat{\tilde{w}}(\phi) \hat{\tilde{v}}(\phi) .
$$

Proof of Lemma I. In Appendix A we prove that $U(t)$ and $S(t)$ are entire in $t$ for all $\varepsilon>0$. A similar argument holds for the $\tilde{V}_{\alpha}(t)$ 's. The region $R_{\phi}$ is defined so that $U_{0}(t)$ is analytic in it. It follows from (3.16) that if $g\left(u_{0}(t)\right) \neq 0$ for all $t \in R_{\phi}$ then $\tilde{V}_{\alpha 0}(t)$ is analytic in this region as well. From the positivity of $g(x)$ on the interval $(0,1)$ we conclude that $g\left(u_{0}(t)\right)$ cannot vanish in an open neighborhood of the interval $[a, A]$. For simplicity, we will further assume that $g\left(u_{0}(t)\right) \neq 0$ in $R_{\phi}$.

$S E$ to Claim 1. An argument similar to the one presented in Appendix A should work. In a neighborhood of the origin, $U_{0}(t) \rightarrow t$ and $U(t) \rightarrow t$; hence $U(t) \rightarrow U_{0}(t)$ there. Continuing in the complex $t$-plane, one can expand $U_{0}(t)$ about an arbitrary point $t^{*} \in R_{\phi}$, and use the same arguments as in Appendix A to show that $U(t)$ must have the same behavior there. A similar argument should work for the $\tilde{V}_{\alpha}$.

$S E$ to Claim 2. It follows from (3.16) and Claim 1 that for $\varepsilon$ sufficiently small and for $t \rightarrow 0, \tilde{v}_{\alpha}(t) \rightarrow 1$; hence, using (3.10) it follows that for $\varepsilon$ and $t$ sufficiently small $\mathbf{V}(t)$ is nonsingular. Since $\mathbf{V}(\Lambda t)=\mathbf{D G}(\mathbf{U}(t)) \mathbf{V}(t)$, and $D G(U(t))$ is analytic and nonsingular (in fact, $\operatorname{det}(D G(U(t)))=1$ ), it follows that $\mathbf{V}(t)$ is nonsingular in $R_{\phi}$. The form of $\mathbf{W}$ follows from (4.23).

$S E$ to Claim 3. It follows from the first two lemmas that if one chooses $r(t)$ to be given by (3.19), with $b_{0}(t)=b_{0}$ of Table 3 , then $r(t)$ is analytic in $R_{\phi}$ and $\delta(\varepsilon, \phi) \rightarrow 0$ as $\varepsilon \rightarrow 0^{+}$.

$S E$ to Claim 4. Since the $z_{\alpha}$ 's are analytic in $\ln (t)$ in $R_{\phi}$, we may use Cauchy's integral formula for the variables $x=\exp ( \pm 2 \pi i \ln (t) / \ln \Lambda)$ to find

$$
z_{\alpha}(t)=\frac{ \pm 1}{\ln \Lambda} \oint_{L} \frac{z_{\alpha}\left(t^{\prime}\right) d\left(\ln \left(t^{\prime}\right)\right)}{1-\exp \left( \pm 2 \pi i \ln \left(t / t^{\prime}\right) / \ln \Lambda\right)}
$$

where $L$ is any closed curve in $R_{\phi}$ which encircles $t$, and the upper (lower) sign stands for $\alpha>0(\alpha<0)$. We choose $L$ as drawn in Figure A.2; in the $\ln (t)$ plane, $L$ has fixed upper and lower legs, $P_{+}$and $P_{-}$, for which the phase of $t^{\prime}$ is fixed at $\phi_{1}$ and $-\phi_{1}$, respectively, and its magnitude $\left|t^{\prime}\right|$ varies between $e^{b}$ and $e^{b+\Lambda}$, where $0<\phi_{1}<\phi, a<b$, and $b+\Lambda<A$. The side legs $P_{s L}$ and $P_{s R}$ are located $\ln \Lambda$ apart along the $\operatorname{Re}\{\ln (t)\}$ axis, and may be deformed so that $L$ encircles $t$.

For $\alpha>0(\alpha<0)$, we define the constants $c_{\alpha, 0}$ as the averaged values of $z_{\alpha}(t)$ along $P_{-}\left(P_{+}\right)$

$$
c_{\alpha, 0}=\frac{1}{\ln \Lambda} \int_{P_{-\operatorname{sign}(\alpha)}} z_{\alpha}\left(t^{\prime}\right) d\left(\ln \left(t^{\prime}\right)\right)
$$

It follows from (D.6) and (D.13) that

$$
c_{\alpha, 0} \leq \xi^{|\alpha| \phi_{1} / \phi_{s}} \delta(\varepsilon, \phi) \hat{\tilde{w}}(\phi) .
$$




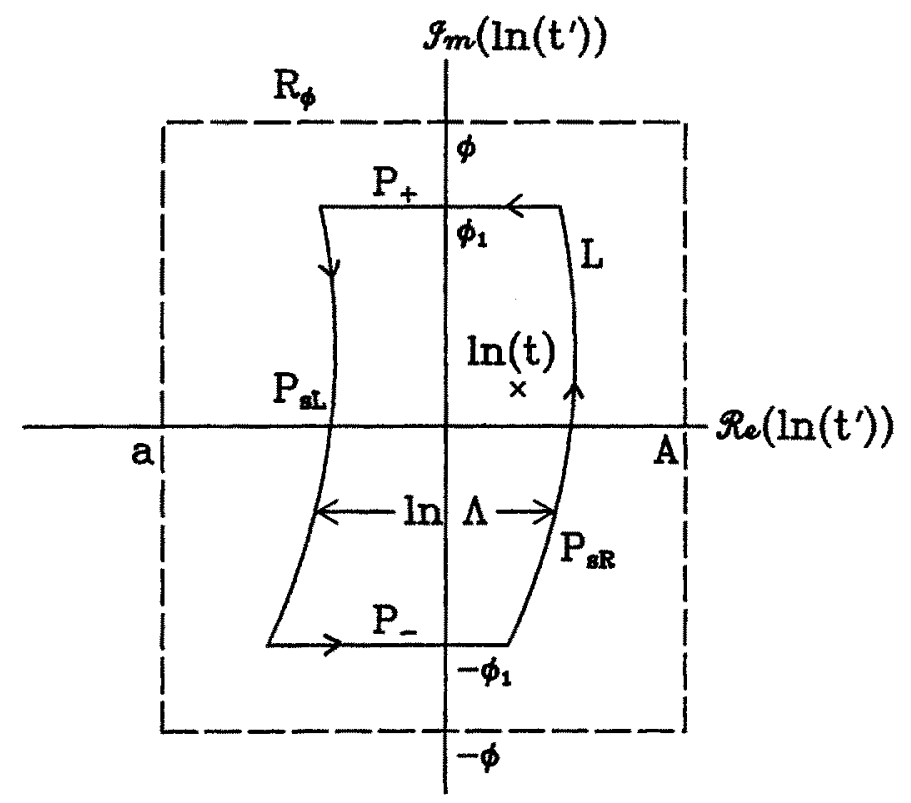

Fig. A.2. The path of integration in the in $t$ plane of (D.12). The legs $P_{ \pm}$are fixed, and the legs $P_{s L}$ and $P_{s R}$ are varied so that $L$ encircles $t$, yet their horizontal distance is always $\ln \Lambda$.

The optimal bound on $c_{\alpha, 0}$ is found by letting $\phi_{1} \rightarrow \phi$, resulting in (D.8).

Now, we estimate $e_{\alpha}(t)=z_{\alpha}(t)-c_{\alpha, 0}$ using (D.12-14). It follows that

$$
e_{\alpha}(t)=E_{\alpha,-}(t)+E_{\alpha,+}(t)+E_{\alpha, s}(t)
$$

where

$$
\begin{aligned}
& E_{\alpha,-}(t)=\frac{\mp 1}{\ln \Lambda} \int_{P_{-}} \frac{z_{\alpha}\left(t^{\prime}\right) d\left(\ln \left(t^{\prime}\right)\right)}{1-\exp \left(-2 \pi i \ln \left(t / t^{\prime}\right) / \ln \Lambda\right)} \\
& E_{\alpha,+}(t)=\frac{ \pm 1}{\ln \Lambda} \int_{P_{+}} \frac{z_{\alpha}\left(t^{\prime}\right) d\left(\ln \left(t^{\prime}\right)\right)}{1-\exp \left(2 \pi i \ln \left(t / t^{\prime}\right) / \ln \Lambda\right)} \\
& E_{\alpha, s}(t)=\frac{1}{\ln \Lambda} \int_{P_{s L}} \frac{\left(z_{\alpha}\left(t^{\prime}\right)-z_{\alpha}\left(\Lambda t^{\prime}\right)\right) d\left(\ln \left(t^{\prime}\right)\right)}{1-\exp \left( \pm 2 \pi i \ln \left(t / t^{\prime}\right) / \ln \Lambda\right)}
\end{aligned}
$$

Using (D.6), we find the following bounds on $E_{\alpha_{t} \pm}(t)$

$$
\begin{aligned}
& E_{\alpha_{s}-}(t) \leq \xi^{\left(q\left(\phi_{1}+\theta\right)+\alpha \phi_{1}\right) / \phi_{s}} \delta(\varepsilon, \phi) \hat{\tilde{w}}(\phi), \\
& E_{\alpha_{*}+}(t) \leq \xi^{\left(q\left(\phi_{1}-\theta\right)-\alpha \phi_{1}\right) / \phi_{s}} \delta(\varepsilon, \phi) \hat{\tilde{w}}(\phi) .
\end{aligned}
$$

Finally, we bound $E_{\alpha, s}(t)$. Using (D.4), (D.16), and (2.8) we find that

$$
\begin{aligned}
& z_{\alpha}(t)-z_{\alpha}(\Lambda t)= \\
& \quad t^{-\chi_{\alpha}}\left[\Delta^{T}(t) \mathbf{F}_{\alpha}\left(U(t), D G^{-1}(U(t)), D^{2} G(U(t))\right) \Delta(t)+O\left(\|\Delta(t)\|^{3}\right)\right],
\end{aligned}
$$


where $\mathbf{F}_{\alpha}\left(U(t), D G^{-1}(U(t)), D^{2} G(U(t))\right)$ is a matrix with bounded entries in $R_{\phi}$. First, we establish that for all $t \in R_{\phi}$,

$$
\begin{aligned}
\Delta(t) & =\sum_{\alpha} c_{\alpha, 0} V_{\alpha}(t)+E_{\Delta}(t) \\
\left\|E_{\Delta}(t)\right\| & \ll\left\|\sum_{\alpha} c_{\alpha, 0} V_{\alpha}(t)\right\| .
\end{aligned}
$$

We multiply (D.12) by $V_{\alpha}(t)$ and sum over $\alpha$. Using (D.7),(D.15), (D.16), and (D.18), we then find an integral equation for $\Delta(t)$ of the form

$$
\begin{aligned}
\Delta(t)= & \sum_{\alpha} V_{\alpha}(t)\left(c_{\alpha, 0}+E_{\alpha,-}(t)+E_{\alpha,+}(t)+O\left(\|\Delta(t)\|^{3}\right)\right) \\
& +\sum_{\alpha} V_{\alpha}(t) \int_{P_{s L}} \frac{t^{-\chi_{\alpha}} \Delta^{T}(t) \mathbf{F}_{\alpha}\left(U(t), D G^{-1}(U(t)), D^{2} G(U(t))\right) \Delta(t)}{1-\exp \left( \pm 2 \pi i \ln \left(t / t^{\prime}\right) / \ln \Lambda\right)}
\end{aligned}
$$

Note that the kernel in the integral is bounded. Since we have an a priori small bound on $\Delta(t)$ in $R_{\phi}$ (Claim 3), we conclude that the integral term is small and can be neglected (this part can be probably justified using a fixed-point type of argument). Finally, using (D.17) we obtain (D.19).

Now, we substitute (D.18) and (D.19) in (D.16c) to establish the bounds on $E_{\alpha, s}$. Using (D.5) and (D.8) we estimate $\Delta(t)$ by $c_{1,0} V_{1}(t)$ when $\theta<0$ and by $c_{-1,0} V_{-1}(t)$ when $\theta>0$, and find

$E_{\alpha, s}(t) \leq \varepsilon^{-1} \xi^{|\alpha| \phi_{1} / \phi_{s}} \delta^{2}\left(\varepsilon, \phi_{1}\right) \hat{\tilde{w}}^{2}(\phi) \varepsilon^{-1}\left[1+\xi^{\left((2-|\alpha|) \phi_{1}-(2|\theta|+\alpha \theta)\right) / \phi_{s}}+\xi^{\left((q-2|\alpha|) \phi_{1} \mp q \theta\right) / \phi_{s}}\right]$.

Equations (D.17) and (D.21), in the limit $\phi_{1} \rightarrow \phi$, result in (D.9).

\section{B. The Distance Function Is Bounded by the Right-hand Side of (1.9)}

Conjecture D.1. Given an entire function $g(x)$ which is positive on $(0,1)$ and which satisfies (1.3) and (1.4), there exist an $\epsilon_{m}$ and an analytic function $r(t)$ such that for $0<\varepsilon<\varepsilon_{m}$ and for real $t$ of order 1

$$
\delta(t) \leq A(\varepsilon) \varepsilon^{\gamma} \xi,
$$

where $\xi$ and $\gamma$ are given by (4.4) and Table 4 respectively, and $A(\epsilon)$ has a weaker dependence than a power law on $\varepsilon$.

Note: In view of the example given by Scheurle et al. ${ }^{23}$, such a conjecture cannot hold in general. We think that the mappings we consider have different properties from theirs (see Section I.C).

Strategy of Proof. We define the family of regions $D_{\psi}$ as $D_{\psi}=R_{\phi_{s}-\varepsilon \psi}$, where $R_{\phi}$ is defined by (D.2). We follow the same logic as in the previous proof, replacing the convergence property of Claim 1 , by an order-of-magnitude statement. 
Step 1. For any $\psi>0$ and $0<\varepsilon<\varepsilon_{m}$, the vector-valued functions $U(t), S(t)$, $\tilde{V}_{\alpha}(t)$, and $\tilde{W}(t)$ are analytic in $D_{\psi}$ and have a unique limit as $\varepsilon \rightarrow 0^{+}$. Moreover, for $\psi$ sufficiently large, these functions are of the same order of magnitude as their limits, on the boundaries of $D_{\psi}$. Namely, $f\left(t_{ \pm \varepsilon \psi}\right)=O\left(f_{0}\left(t_{ \pm \varepsilon \psi}\right)\right)$, where

$$
t_{ \pm \varepsilon \psi}=\left|t_{ \pm \varepsilon \psi}\right| \exp \left( \pm i\left(\phi_{s}-\varepsilon \psi\right)\right) .
$$

Step 2. There exists a function $r(t)$, analytic in $D_{\psi}$, such that $r(\Lambda t)=r(t) / \Lambda$ and such that for all $t \in D_{\psi}$,

$$
\delta(t) \leq \tilde{K}_{1}\left\|U_{0}\left(t_{\varepsilon \psi}\right)\right\|
$$

for some constant $\tilde{K}_{1}$.

Corollary 1. $z_{\alpha}(t)=(\mathrm{W} \Delta(t))_{\alpha}$ are analytic in $D_{\psi}$.

Corollary 2. The following bounds hold on the boundaries of $D_{\psi}$ :

$$
\begin{aligned}
& \left|v_{\alpha}\left(t_{ \pm \varepsilon \psi}\right)\right| \leq \tilde{K}_{2}(\varepsilon) \xi^{ \pm \alpha} \tilde{v}_{\alpha 0}\left(t_{ \pm \varepsilon \psi}\right), \\
& \left|w_{\alpha}\left(t_{ \pm \varepsilon \psi}\right)\right| \leq \tilde{K}_{3}(\varepsilon) \xi^{\mp \alpha} \tilde{w}_{\alpha 0}\left(t_{ \pm \varepsilon \psi}\right),
\end{aligned}
$$

where $\tilde{K}_{2}(\varepsilon)$ and $\tilde{K}_{3}(\varepsilon)$ have a weaker dependence on $\varepsilon$ than a power law.

Corollary 3. On the boundaries of $D_{\psi}$

$$
\left|z_{\alpha}\left(t_{ \pm \varepsilon \psi}\right)\right| \leq \tilde{K}_{1} \tilde{K}_{3}(\varepsilon) \xi^{\mp \alpha}\left\|U_{0}\left(t_{ \pm \varepsilon \psi}\right)\right\|\left\|\tilde{W}_{\alpha 0}\left(t_{\varepsilon \psi}\right)\right\| .
$$

Step 3. For $\alpha \neq q / 2$ and for a "generic" $g(x)$ (see Conjecture 1) there exist constants $c_{\alpha, 0}$ such that

$$
\begin{aligned}
z_{\alpha}(t) & =c_{\alpha, 0}+e_{\alpha}(t), \\
\left|c_{\alpha, 0}\right| & \approx K_{\alpha}(\varepsilon) \xi^{|\alpha|}\left\|U_{0}\left(t_{ \pm \varepsilon \psi}\right)\right\|\left\|\tilde{W}_{\alpha 0}\left(t_{\varepsilon \psi}\right)\right\|, \\
\left|e_{\alpha}(t)\right| & <\left|c_{\alpha, 0}\right| \xi^{\varepsilon \psi(2-|\alpha|) / \phi_{s}},
\end{aligned}
$$

where the $K_{\alpha}(\varepsilon)$ 's have a weaker dependence than a power law on $\varepsilon$.

Therefore, for real $t$ and $\alpha= \pm 1$ the $z_{\alpha}$ 's are small and are constants to exponential order in $\psi$. Moreover, for real $t,\left|z_{\alpha}(t)\right| \ll\left|z_{1}\right|$ for $|\alpha|>1$. Therefore, using (D.4), (D.5), (D.27), (D.28), and (D.29) we obtain (D.22), with

$$
A(\varepsilon)=\left(K_{1}(\varepsilon)+K_{-1}(\varepsilon)\right)\left\|U_{0}\left(t_{ \pm \varepsilon \psi}\right)\right\|\left\|\tilde{W}_{\alpha 0}\left(t_{\varepsilon \psi}\right)\right\| .
$$

\section{References}

1. C. Bender and S. Orszag, Advanced Mathematical Methods for Scientists and Engineers, McGraw-Hill, New York (1978).

2. J. Kevorkian and J. D. Cole, Perturbation Methods in Applied Mathematics, Springer Verlag, New York (1981).

3. M. Feingold, L. P. Kadanoff, and O. Piro, Passive Scalars, Three-Dimensional Volume Preserving Maps, and Chaos, J. Stat. Phys. 50, 529-565 (1988). 
Beyond All Orders: Singular Perturbations in a Mapping

4. J. Guckenheimer and P. Holmes, Non-Linear Oscillations, Dynamical Systems and Bifurcations of Vector Fields, Springer-Verlag, New York (1983).

5. S. Wiggins, Global Bifurcations and Chaos-Analytical Methods, Springer-Verlag, New York (1988).

6. M. W. Hirsch, C. C. Pugh, and M. Shub, Invariant Manifolds, Springer Lecture Notes in Mathematics, Vol. 583. Springer-Verlag, New York (1977).

7. In fact, after $U(t)$ (resp. $S(r)$ ) spirals away from the fixed point 1 (resp. 0 ) it may come back arbitrarily close to the same fixed point. The minima we are after is the global minima associated with the first spiraling, namely, we minimize $\|U(t)-1\|($ resp. $\|S(r)-0\|$ ) over a finite interval of $t$ (resp. $r$ ). In practice, it is not difficult to determine the appropriate interval of minimization, as described in Section IV.E.

8. The definition of $\delta_{0}$ and $\delta_{1}$ for two-dimensional maps $(q=2)$ is given in Section IV.C and is illustrated in Figure 9. In this case the stable and unstable curves cross an infinite number of times; hence the usual definition of $\delta_{0}$ and $\delta_{1}$ gives the trivial result.

9. For example, on the disappearance of solitons and the transient behavior of solutions due to separatrix breaking in the Sine-Gordon equation, see A. Bishop, D. W. McLaughlin, M. G. Forest, and E. Overman II, Quasi-periodic Route to Chaos in a Near-Integrable PDE: Homoclinic Crossings, Phys. Lett. A 127, 335-340 (1988).

10. Numerous papers on the subject of transport in phase space of two-dimensional maps due to separatrix breaking were written in the past few years. One example, in which references to previous work can be found, is the paper by V. Rom-Kedar and S. Wiggins, Transport in Two-Dimensional Maps, Arch. Rational Mech. Anal. 109, 239-298 (1990).

11. M. J. Ablowitz and B. M. Herbst, On Homoclinic Structure and Numerically Induced Chaos for the Nonlinear Schrödinger Equation, SIAM J. Appl. Math. 50, 339-351 (1990).

12. K. Hockett, Chaotic Numerics from an Integrable Hamiltonian System, Proc. Amer. Math. Soc. 108, 271-281 (1990).

13. H. Poincaré, Sur les équations de la dynamique et le problème de trois corps, Acta Math., 13, 1-270 (1890).

14. N. N. Nekhoroshev, An Exponential Estimate of the Time of Stability of Near-Integrable Hamiltonian Systems, Russian Math. Surveys 32, 1-65 (1977).

15. A. Neishtadt, The Separation of Motions in Systems with Rapidly Rotating Phase, P.M.M. USSR 48, 133-139 (1984).

16. P. Holmes, J, Marsden, and J, Scheurle, Exponentially Small Splittings of Separatices with Applications to KAM Theory and Degenerate Bifurcations, Cont. Math. 81, 213-244 (1988).

17. G. M. Zaslavskii, R. Z. Sagdeev, D. A. Usikov, and A. A. Chernikov, Minimal Chaos, Stochastic Webs and Structures of Quasicrystal Symmetry, Sov. Phys. Usp. 31 (10), (1988); and references therein.

18. S. Tanveer, private communication.

19. M. Kruskal and H. Segur, Princeton Aeronautical Lab. Report (1986).

20. C. J. Amick and J. B. McLeod, A Singular Perturbation Problem in Needle Crystals, Arch. Rational Mech. Anal. 109, 139-171 (1990).

21. V. F. Lazutkin, 1. G. Schachmannski and M. B. Tabanov, Splitting of Separatrices for Standard and Semistandard Mappings, Physica D 40, 235-248 (1989).

22. E. Fontich and C. Simó, The Splitting of Separatrices for Analytic Diffeomorphisms, Ergod. Th. \& Dynam. Sys. 10, 295-318 (1990).

23. J. Scheurle, J. E. Marsden, and P. Holmes, Exponentially Small Estimates for Separatrix Splittings. To appear in the proceedings of the NATO Advanced Workshop on Asymptotics Beyond All Orders, eds. S. Tanveer and H. Segur (1991).

24. The heuristic arguments of Appendix $D$ and the numerical error analysis of Appendix $\mathrm{C}$ imply that the quadratic error terms are of order $\left|c_{1,0}\right|^{2}$; hence our order-of-magnitude estimates for $c_{\alpha}$ for $|\alpha| \geq 3$ and our assertions regarding its $t$-dependence for $|\alpha| \geq 2$ may be in error. The feature we use, which is definitely correct, is that $c_{\alpha} \leq O\left(\left|c_{1,0}\right|^{2}\right)$ for $\alpha \geq 2$.

25. See, for example, E. Hille, Analytic Function Theory, Vol. 2, Section 14, Chelsea (1977). 